\title{
Transmissionselektronenmikroskopische Untersuchungen zur Koausscheidung von Übergangselementen in kristallinem Silizium
}

\author{
Dissertation \\ zur Erlangung des Doktorgrades \\ der Mathematisch-Naturwissenschaftlichen Fakultäten \\ der Georg-August-Universität zu Göttingen
}

\author{
vorgelegt von \\ Carsten Rudolf \\ aus \\ Hameln
}

Göttingen 2009 
D 7

Referent: Herr Prof. Dr. M. Seibt

Korreferent: Herr Prof. Dr. H. Hofsäß

Tag der mündlichen Prüfung: 24.2.2009 


\section{Inhaltsverzeichnis}

1. Einleitung 1

2. Einführung 5

2.1. Eigenschaften von Übergangselementen in $\mathrm{Si}$. . . . . . . . . . . . . . 5

2.1.1. Löslichkeit und Diffusionskoeffizient in $\mathrm{Si}$. . . . . . . . . . . 5

2.1.2. Metallsilizid-Ausscheidungen in $\mathrm{Si} \ldots \ldots \ldots$. . . . . . . . . . . . . . . . . . . . .

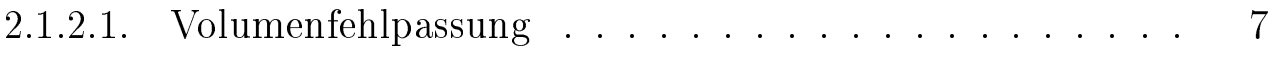

2.1.2.2. Nickel . . . . . . . . . . . . . . . . . . . 10

2.1.2.3. Kupfer und Palladium . . . . . . . . . . . . . . . 11

2.2. Ternäre Phasen aus Si und zwei Metallen . . . . . . . . . . . . . 12

2.2.1. $\mathrm{Cu}-\mathrm{Ni}-\mathrm{Si} \ldots \ldots \ldots \ldots \ldots \ldots \ldots$

2.2.2. Ni-Pd-Si . . . . . . . . . . . . . . . 14

2.3. Koausscheidung von Übergangsmetallen . . . . . . . . . . . . . . . . 15

3. Methoden 17

3.1. Transmissionselektronenmikroskopie $(\mathrm{TEM}) \ldots \ldots \ldots \ldots \ldots$

3.2. Energiedispersive Röntgenspektroskopie (EDX) . . . . . . . . . . . . 17

4. Ergebnisse 21

4.1. Ausscheidungen nach Diffusion von Kupfer . . . . . . . . . . . . . . . 21

4.2. Ausscheidungen nach Kodiffusion von Kupfer und Nickel . . . . . . . . . 25

4.2.1. Ausscheidungen unter kupferreichen Bedingungen . . . . . . . 25

4.2.1.1. Verteilung . . . . . . . . . . . . 26

4.2.1.2. Struktur . . . . . . . . . . . . . 28

4.2.1.3. Zusammensetzung . . . . . . . . . . . . . 35

4.2.2. Ausscheidungen unter nickelreichen Bedingungen . . . . . . . . 44

4.2.3. Zusammenfassung . . . . . . . . . . . . . . . . 51

4.3. Ausscheidungen nach Kodiffusion von Palladium und Nickel . . . . . . . 52 
4.3.1. Verteilung . . . . . . . . . . . . . . . . 52

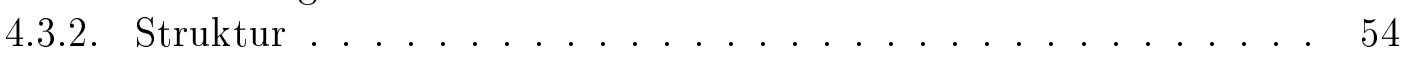

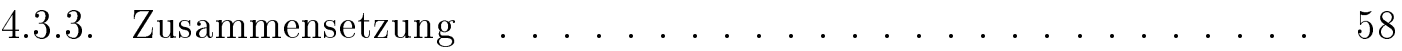

5. Zusammenfassung und Diskussion 65

5.1. Verteilung von Metallsilizidausscheidungen im Bikristall . . . . . . . . 65

5.1.1. Zusammenfassung . . . . . . . . . . . . . . . . . 65

5.1.2. Diskussion . . . . . . . . . . . . . . . . 66

5.2. Koausscheidung zweier metallischer Verunreinigungen . . . . . . . . 69

5.2.1. Zusammenfassung . . . . . . . . . . . . . . . . . . . . 69

5.2.1.1. Kupferreiche Bedingungen . . . . . . . . . . . . . 70

5.2.1.2. Nickelreiche Bedingungen _. . . . . . . . . . . 70

5.2.2. Diskussion . . . . . . . . . . . . . . . . . 71

5.2.2.1. Einfluss des Konzentrationsverhältnisses . . . . . . . 71

5.2.2.2. Gitterplatz von $\mathrm{Cu}$ und Volumenfehlpassung . . . . . . . 73

5.2.2.3. Vergleich von $\mathrm{NiSi}_{2}: \mathrm{Cu}$ - und $\mathrm{NiSi}_{2}$-Ausscheidungen . . . 76

5.2.2.4. Konzentration von $\mathrm{Cu}$ in $\mathrm{NiSi}_{2}: \mathrm{Cu}$-Ausscheidungen . . . 77

5.2.2.5. Ausblick: Gitterplatzbestimmung mit TEM . . . . . . 78

5.2.2.6. Ausblick: Koausscheidung in Si für die Photovoltaik . . . 79

Anhänge

A. Herstellung von Si-Bikristallen 83

B. Probenpräparation 87

B.1. Präparation der Diffusionsproben . . . . . . . . . . . 87

B.2. Präparation von Querschnittsproben . . . . . . . . . . . . 89

B.2.1. Proben für Ätzgrübchenuntersuchung . . . . . . . . . . . . . 89

B.2.2. TEM-Proben . . . . . . . . . . . . . . . 90

C. Volumenfehlpassung bei Vorliegen zweier metallischer Verunreinigungen 91

\begin{tabular}{ll} 
Literaturverzeichnis & 95 \\
\hline
\end{tabular}

$\begin{array}{ll}\text { Danksagung } & 103\end{array}$

$\begin{array}{ll}\text { Lebenslauf } & 105\end{array}$ 
$\Gamma_{\text {Kapitel }} 1$

\section{Einleitung}

Unsere Zivilisation steht vor der Herausforderung, zur Gewinnung nutzbarer Energie neue nachhaltige Quellen zu erschließen, anstelle auf den Verbrauch begrenzter Ressourcen zu setzen, insbesondere der fossilen Energieträger. Eine nahezu unerschöpfliche Quelle stellt die Wind- und Sonnenenergie dar. Der Erzeugung elektrischer Energie aus Licht, der Photovoltaik, kommt dabei eine Schlüsselrolle zu, da sie erhebliches Kostenoptimierungspotential durch den Übergang zur Massenproduktion hat und die Möglichkeit zum Aufbau einer dezentralen Stromversorgung bietet. Das Zusammenwirken universitärer Forschung und industrieller Entwicklung kann hier einen wichtigen Beitrag leisten.

Wie die Mikroelektronik ist die Photovoltaik eine Halbleitertechnologie, die auf der Verwendung vorwiegend von Silizium als Rohstoff basiert [1]. In beiden Fällen ist der Fortschritt durch die Kontrolle der Defekte und Verunreinigungen in Silizium beeinflusst. Die Mikroelektronik bedient sich dazu kostenintensiver Verfahren der Kristallzucht zur Herstellung von einkristallinem praktisch versetzungsfreiem Silizium [2] und ergreift aufwendige Reinhaltungsmaßnahmen, um Verunreinigungen zu vermeiden. Zudem wird nur eine oberflächennahe Schicht in extrem gutem Zustand benötigt, so dass bei einer unbeabsichtigten Kontamination Fremdatome in das Kristallvolumen umverteilt werden können [3, 4]. Die Photovoltaik nimmt Defekte und Verunreinigungen im Ausgangsmaterial in einem sehr viel höheren Ausmaß in Kauf. Bei der Herstellung von mehr als 50\% der Solarzellen kommt kostengünstiges so genanntes multikristallines Silizium (mc-Si) zum Einsatz [1, 5]. Es enthält nicht nur kristallographische Defekte wie Korngrenzen und Versetzungen, sondern auch nichtmetallische Verunreinigungen wie Sauerstoff und Kohlenstoff [6] und metallische Verunreinigungen, unter denen Eisen, Kupfer und Nickel am häufigsten sind [5, 7].

Metallische Fremdatome üben einen schädlichen Einfluss auf den Wirkungsgrad einer Solarzelle aus, da sie mit diskreten Zuständen verbunden sind, die energetisch tief 
in der Bandlücke von Silizium liegen [8] und die Rekombinationsrate von Elektronen und Löchern aus Leitungs- und Valenzband gegenüber defektfreiem Silizium um mehrere Größenordnungen erhöhen [9, 10]. Ausscheidungen von Metallsilizidphasen zeigen zum Teil eine sehr hohe Rekombinationsaktivität [11], die auf die Existenz von Raumladungszonen an den Metallsilizid-Silizium-Grenzflächen hinweist [12]. Zwischen Fremdatomen und kristallographischen Defekten in Silizium sind Wechselwirkungen möglich. An Versetzungen beispielsweise können Fremdatome sich chemisch binden [13], sich in deren Verzerrungsfeld aufgrund elastischer Wechselwirkung anreichern [14] oder sich ausscheiden [14]. Durch Verunreinigung mit metallischen Fremdatomen erhöht sich die Rekombinationsaktivität von Versetzungen drastisch [15-17]. In multikristallinem Silizium kommt es durch Wechselwirkung mit metallischen Verunreinigungen außerdem zu einer Erhöhung der Rekombinationsaktivität von Korngrenzen [18-20].

Die Photovoltaik versucht im Rahmen der zur Herstellung einer Solarzelle erforderlichen Prozesse die metallischen Verunreinigungen in inaktive Bereiche der Zelle umzuverteilen [21] oder so zu behandeln, dass ihr schädlicher Einfluss reduziert wird. Ein Ansatz dazu ist die so genannte Defektmanipulation, im englischen Sprachgebrauch auch „defect engineering“. Die metallischen Fremdatome sollen in wenigen großen möglichst weit voneinander entfernten Ausscheidungen gesammelt werden, so dass der mittlere Abstand zwischen den Rekombinationszentren wächst und sich die Diffusionslänge der Minoritätsladungsträger erhöht [22]. Physikalisch finden dabei Prozesse wie beispielsweise Ostwaldreifung von Ausscheidungen, heterogene Keimbildung und Segregation von Fremdatomen aus Si in Ausscheidungen anderer Phasen statt.

Das Konzept der Defektmanipulation wird im Zusammenhang mit dem Vorhaben diskutiert, so genanntes verbessertes metallurgisches Silizium, im Englischen als „upgraded metallurgical silicon“ (umg-Si) oder „solar-grade silicon“ (SoG-Si) bezeichnet, als Ausgangsmaterial für Solarzellen zu verwenden [22-24]. Dieses Material ist ebenfalls multikristallin, es enthält jedoch wesentlich höhere Konzentrationen an metallischen und weiteren Verunreinigungen als herkömmliches mc-Si [24].

Multikristallines Si ist typischerweise mit einer Vielzahl von Fremdelementen gleichzeitig verunreinigt. Das Ausscheidungsverhalten einzelner Übergangselemente in Silizium jeweils als alleinige Verunreinigung ist gut bekannt [25-27]. Heuer et al. und Buonassisi et al. wiesen jedoch vor Kurzem darauf hin [28, 29], dass bisher wenige Kenntnisse zur Koausscheidung zweier oder mehrerer gleichzeitig in Silizium anwesender metallischer Verunreinigungen vorliegen $[30-32]$. Ihre Untersuchungen mittels ortsaufgelöster röntgenspektroskopischer Verfahren von Ausscheidungen in gleichzeitig mit $\mathrm{Fe}, \mathrm{Cu}$ und $\mathrm{Ni}$ kontaminiertem Silizium, die sich unter Bedingungen ähnlich wie bei der Kristallzucht während sehr langsamer Abkühlung gebildet haben, zeigen eine Anhäufung der verschiedenen Verunreinigungsatome an denselben Stellen [28, 29]. Unterschiede in Form, Größe und Verteilung der Ausscheidungen in thermisch anders behandelten Proben lassen sich 
nach Ansicht der Autoren auf die Bildung von Ausscheidungen einer quaternären Mischphase bei hoher Temperatur zurückführen.

Die vorliegende Arbeit entstand im Rahmen des vom BMU geförderten Verbundprojektes SolarFocus, das die Wechselwirkungen verschiedener photovoltaik-relevanter Defekte in kristallinen Siliziummaterialien erforscht. Sie untersucht mit Methoden der Transmissionselektronenmikroskopie (TEM) die strukturellen und chemischen Eigenschaften von Metallsilizidausscheidungen jeweils zweier gleichzeitig anwesender metallischer Verunreinigungen, die sich nach Eindiffusion unter Bedingungen ähnlich zu Hochtemperaturschritten im Rahmen der Solarzellenherstellung gebildet haben. Die gefundenen Eigenschaften der Ausscheidungen werden mit dem Konzentrationsverhältnis der Verunreinigungen in Beziehung gesetzt. Als Ausgangsmaterial dienten Silizium-Bikristalle, die aus zwei mittels des Waferbondingverfahrens [33] miteinander verbundenen einkristallinen Scheiben bestehen. Im Bikristall finden sich die verschiedenen Defektarten, die im mc-Si vorkommen, in einer einfachen räumlichen Anordnung wieder, so dass ihre Konkurrenz in der Verteilung metallischer Fremdatome und ihrer Ausscheidungen experimentell zugänglich wird. Das Versetzungsnetzwerk an der Grenzfläche der beiden Scheiben entspricht einer Kleinwinkelkorngrenze. Eine der beiden Scheiben enthält Mikrodefekte, bei denen es sich Siliziumoxidteilchen und Versetzungsringe handelt, während die andere Scheibe nahezu defektfrei ist.

Es wurden Ausscheidungen von Kupfer, Nickel und Palladium entweder als alleinige metallische Verunreinigung $(\mathrm{Cu})$ oder in Kombination $(\mathrm{Cu}$ und $\mathrm{Ni}, \mathrm{Pd}$ und $\mathrm{Ni}$ ) untersucht. Diese Elemente besitzen im binären Metall-Silizium-System bei den in dieser Arbeit verwendeten Temperaturen von $900^{\circ} \mathrm{C}$ und $1050^{\circ} \mathrm{C}$ im Vergleich zu anderen metallischen Verunreinigungen hohe Löslichkeiten in Silizium und scheiden sich aufgrund hoher Diffusionskoeffizienten bei langsamer Abkühlung (diese Arbeit: -6K/s) annähernd vollständig aus, so dass sie sich experimentell mittels TEM gut untersuchen lassen.

1. Die Verteilung von Kupfer als metallischer Verunreinigung im Volumen ist unter Bedingungen der Konkurrenz mit einer Korngrenze und einer freien Oberfläche durch Mikrodefekte wie Oxidausscheidungen und Versetzungsringe bestimmt.

2. Auch bei Anwesenheit von Mikrodefekten lässt sich unter für den Solarzellenprozess typischen Abkühlbedingungen Kupfer effizient in eine Schicht an der Oberfläche umverteilen, die aus der mit Silizium im Gleichgewicht stehenden Phase besteht.

3. Nach Kodiffusion von Kupfer und Nickel hängen Form, Größe und Verteilung der Ausscheidungen vom Konzentrationsverhältnis der beiden Verunreinigungen ab. Unter kupferreichen Bedingungen liegen von Versetzungen berandete Kolonien kleiner Teilchen vor wie nach der Ausscheidung von Kupfer als alleiniger metallischer Verunreinigung, unter nickelreichen Bedingungen dagegen bilden sich einzelne große Ausscheidungen, wie typischerweise für Nickel allein in Silizium. 
Nach Kodiffusion von Nickel und Palladium, das als alleinige Verunreinigung in Silizium ein ähnliches Ausscheidungsverhalten aufweist wie Kupfer [25], aber eine geringere Löslichkeit in Silizium hat als Nickel, ergeben sich Ausscheidungen mit vergleichbaren Eigenschaften wie nach Kodiffusion von Kupfer und Nickel unter nickelreichen Bedingungen.

Die Ergebnisse dieser Arbeit legen nahe, dass das Konzentrationsverhältnis der beteiligten Verunreinigungen ein geeignetes ordnendes Kriterium für die auftretenden Defekte ist.

4. Die Ausscheidungen lassen sich den aus den binären Systemen bekannten Gleichgewichtsphasen $\mathrm{Cu}_{3} \mathrm{Si}, \mathrm{NiSi}_{2}$ und $\mathrm{Pd}_{2} \mathrm{Si}$ zuordnen. Unabhängig vom System und vom Konzentrationsverhältnis der metallischen Komponenten kommt es zur Bildung zusammenhängender Ausscheidungen unterschiedlicher Phasen und zur Lösung der jeweils anderen metallischen Verunreinigung. Die Phasen der Ausscheidungen nach Kodiffusion werden hier als $\mathrm{Cu}_{3} \mathrm{Si}$ :Ni und $\mathrm{NiSi}_{2}$ :Cu bzw. $\mathrm{NiSi}_{2}: \mathrm{Pd}$ bezeichnet.

Unterschiede zwischen der Konzentration von Kupfer in $\mathrm{NiSi}_{2}: \mathrm{Cu}$-Ausscheidungen nach Kodiffusion und der aus einem isothermischen Schnitt des ternären Phasendiagramms Cu-Ni-Si [34] extrapolierten Löslichkeit lassen sich als Folge der unterschiedlichen thermischen Behandlung erklären, wobei prinzipiell auch zusätzliche Beiträge zur freien Enthalpie des System zu berücksichtigen sind, die sich aus der Volumenfehlpassung zwischen $\mathrm{Nisi}_{2}: \mathrm{Cu}$ und Si ergeben.

5. Form, Größe und Verteilung der $\mathrm{Cu}_{3} \mathrm{Si}$ :Ni-Teilchen unter kupferreichen Bedingungen und der $\mathrm{NiSi}_{2}: \mathrm{Cu}, \mathrm{Pd}$-Ausscheidungen unter nickelreichen Bedingungen sind kompatibel zum bekannten Ausscheidungsverhalten von Kupfer oder Nickel in Silizium. Unter kupferreichen Bedingungen hingegen liegen $\mathrm{NiSi}_{2}: \mathrm{Cu}$-Teilchen in einer Größe und Anordnung vergleichbar der von $\mathrm{Cu}_{3}$ Si-Ausscheidungen vor. Dieses Verhalten ist konsistent zu der Annahme, dass Kupferatome in der $\mathrm{NiSi}_{2}: \mathrm{Cu}-\mathrm{Phase}$ Silizium-Plätze besetzen. 
Kapitel 2

\section{Einführung}

\subsection{Eigenschaften von Übergangselementen in $\mathrm{Si}$}

\subsubsection{Löslichkeit und Diffusionskoeffizient in Si}

Einen Überblick über die Eigenschaften metallischer Verunreinigungen in Silizium bei hohen Temperaturen sowie über Keimbildungs- und Wachstumsmechanismen der Ausscheidungen geben Schröter et al. in [27]. Die 3d-Übergangsmetalle $\mathrm{Co}, \mathrm{Ni}$ und $\mathrm{Cu}$, sowie das 4d-Übergangselement Pd sind die am schnellsten in Silizium diffundierenden metallischen Verunreinigungen. Dieses lässt sich verstehen, wenn man beachtet, dass die Atome der 3d-Übergangsmetalle in Silizium vorwiegend interstitiell vorliegen, während andere metallische Fremdatome wie beispielsweise die des 5d-Übergangselementes Gold substitutionell eingebaut sind. Innerhalb der 3d-Reihe wiederum haben $\mathrm{Cu}$ und $\mathrm{Ni}$ die niedrigsten Werte der Wanderungsenthalpie aller Metalle, was sich nach Utzig [35] durch eine geringere elastische Verzerrung infolge eines kleineren Atomradius begründen lässt, der von Ti nach $\mathrm{Cu}$ montoton abnimmt. Mittels der Wanderungsenthalpie $H_{m i}$ lässt sich der Diffusionskoeffizient wie folgt ausdrücken:

$$
D=D_{0} \exp \left(-\frac{H_{m i}}{k_{B} T}\right)
$$

wobei $D_{0}$ ein Vorfaktor ist. Für die in dieser Arbeit betrachteten Metalle $\mathrm{Cu}, \mathrm{Ni}$ und $\mathrm{Pd}$ sind Werte für $H_{m i}$ und $D_{0}$ in der Tabelle 2.1 zusammengestellt.

Die Löslichkeit einer metallischen Verunreinigung in Si ist definiert als die Konzentration des Metalls in Silizium im thermodynamischen Gleichgewicht mit einer im Phasendiagramm benachbarten Phase. Das Phasendiagramm des binären Systems aus Si und 


\begin{tabular}{cccl} 
Metall & $D_{0}\left(\mathrm{~cm}^{2} / \mathrm{s}\right)$ & $H_{m i}(\mathrm{eV})$ & Referenz \\
\hline $\mathrm{Cu}$ & $5 \cdot 10^{-3}$ & 0.4 & Hall und Racette [36] \\
$\mathrm{Ni}$ & $2 \cdot 10^{-3}$ & 0.47 & Bakhadyrkhanov et al. [37] \\
$\mathrm{Pd}$ & $8 \cdot 10^{-2}$ & 0.89 & Graff et al. [38]
\end{tabular}

Tabelle 2.1. - Parameter des Diffusionskoeffizienten in Si für $\mathrm{Cu}, \mathrm{Ni}$ und $\mathrm{Pd}$

einem 3d-Übergangselement $M$ enthält typischerweise Metallsilizide $M_{x} S i_{y}$. Es gibt in einem binären Phasendiagramm genau einen, entweder eutektischen oder peritektischen Punkt, an dem die Lösung metallischer Fremdatome in Silizium, das siliziumreichste Metallsilizid und die flüssige Phase im Gleichgewicht stehen. Unterhalb der eutektischen $\left(T_{\text {eut }}\right)$ oder peritektischen Temperatur $\left(T_{\text {per }}\right)$ dieses Punktes steht die Lösung metallischer Fremdatome in Silizium im Gleichgewicht mit dem Silizid. Für diesen Fall lässt sich für die Löslichkeit einer metallischen Verunreinigung $M$ in Silizium ein Arrhenius-Gesetz mit einer Aktivierungsenergie $Q_{M}$ angeben

$$
[M]_{s o l}=k_{M} \exp \left(-\frac{Q_{M}}{k_{B} T}\right),
$$

wobei der Vorfaktor sich durch die Dichte der Zwischengitterplätze in Si und einem weiteren Parameter $S_{M}$ ausdrücken lässt: $k_{M}=5 \cdot 10^{22} \exp \left(\frac{S_{M}}{k_{B}}\right) \mathrm{cm}^{-3}$. Thermodynamisch entsprechen die Parameter $Q_{M}$ und $S_{M}$ der partiellen Exzessenthaphie und -entropie von $M$-Atomen in Silizium gegenüber $M$-Atomen in der Silizid-Phase, wobei die partielle freie Exzessenthaphie $Q_{M}-T S_{M}$ die Abweichung des chemischen Potentials der $M$-Atome in Si vom chemischen Potential einer idealen Lösung angibt. Die folgende Tabelle enthält die Löslichkeitsparameter für Cu, Ni und Pd in Si sowie die Stöchiometrie der jeweiligen Gleichgewichtsphase und die eutektische oder peritektische Temperatur.

\begin{tabular}{c|ccc|ccc}
$M$ & $\begin{array}{c}S_{M} \\
\left(k_{B}\right)\end{array}$ & $\begin{array}{c}Q_{M} \\
(\mathrm{eV})\end{array}$ & Referenz & Silizid & $\begin{array}{c}\text { eut./per. } \\
(\mathrm{e}, \mathrm{p})\end{array}$ & $\begin{array}{c}T_{\text {eut,per }} \\
\left({ }^{\circ} \mathrm{C}\right)\end{array}$ \\
\hline $\mathrm{Cu}$ & 2.4 & 1.49 & Weber [39] & $\mathrm{Cu}_{3} \mathrm{Si}$ & $\mathrm{e}$ & 802 \\
$\mathrm{Ni}$ & 3.2 & 1.68 & Weber [39] & $\mathrm{NiSi}_{2}$ & $\mathrm{p}$ & 993 \\
$\mathrm{Pd}$ & 1.2 & 1.64 & Frank [40] & $\mathrm{Pd}_{2} \mathrm{Si}$ & $\mathrm{e}$ & 892
\end{tabular}

Tabelle 2.2. - Löslichkeitparameter, Gleichgewichtsphase und eutektische oder peritektische Temperatur für $\mathrm{Cu}, \mathrm{Ni}$ und $\mathrm{Pd}$

Oberhalb der eutektischen oder peritektischen Temperatur steht die Lösung metallischer Fremdatome in Silizium im Gleichgewicht mit der flüssigen Phase. In diesem Fall hängt die Löslichkeit von $M$ in Si nicht nur von der partiellen freien Exzessenthalpie der $M$-Atome in Si gegenüber $M$-Atomen in der Flüssigkeit sondern zusätzlich noch vom 
Metallanteil der Flüssigkeit ab, der entsprechend der Liquiduslinie im Phasendiagramm variiert. Insbesondere nimmt bei sehr hohen Temperaturen die Löslichkeit wieder ab. Dabei liegt bei allen Übergangselementen die Temperatur der maximalen Löslichkeit deutlich über der jeweiligen eutektischen oder peritektischen Temperatur, was als retrograde Löslichkeit bezeichnet wird und sich auf die hohe partielle Exzessenthalphie der $M$-Atome in Si gegenüber der Flüssigkeit zurückführen lässt [41]. Abb. 2.1 zeigt den Verlauf der Löslichkeit, der sich so für verschiedene metallische Verunreinigungen $(\mathrm{Cu}$, Ni und Pd) ergibt [27].

Die Löslichkeit der Übergangsmetalle in Si ist thermisch stark aktiviert. Daher besteht bei Abkühlung einer bei hoher Temperatur gesättigten Lösung eine starke treibende Kraft für metallische Verunreinigungen sich auszuscheiden. Bei Abkühlung mit einer Rate von $4 \mathrm{~K} / \mathrm{s}$ führt die Kombination aus starker treibender Kraft und hoher Mobilität dazu, dass bei Raumtemperatur die meisten metallischen Verunreinigungen in Si in Form von Metallsilizid-Ausscheidungen vorliegen [26].

\subsubsection{Metallsilizid-Ausscheidungen in Si}

Bei Vorliegen einer einzelnen metallischen Verunreinigung $M$ in Silizium bestehen die Ausscheidungen aus der Phase, mit der die Lösung von $\mathrm{M}$ in Si nach dem entsprechenden binären Phasendiagramm im Gleichgewicht steht. Enthält das Phasendiagramm intermetallische Phasen, was bei den binären System aus Silizium und einem Übergangsmetall mit der Ausnahme von Gold der Fall ist, handelt es sich bei der Gleichgewichtsphase um das siliziumreichste Metallsilizid des jeweiligen Systems. Diese Silizide sind für die in der vorliegenden Arbeit betrachteten Metalle in Tab. 2.2 angegeben.

Bei der Bildung von Metallsilizidausscheidungen in Si nach langsamer Abkühlung mit $\dot{T}=-4 \mathrm{~K} / \mathrm{s}$ ergeben sich für verschiedene 3d-Übergangsmetalle zwei sehr unterschiedliche Mikrostrukturen. Im Fall von $\mathrm{Cu}$ lassen sich ausgedehnte Kolonien vieler kleiner Ausscheidungen beobachten [14, 25, 42-44]. Eine ähnliche Morphologie ist auch für Ausscheidungen von $\mathrm{Pd}$ in $\mathrm{Si}$ zu finden [25]. Dagegen kommt es im Fall von Fe, Co und Ni zur Bildung einzelner stabförmiger (Fe [30, 45]), plättchenförmiger (Co, Ni [25]) oder polyedrischer Ausscheidungen (Ni [25]). Während sich Unterschiede bei Größe und Dichte der Ausscheidungen durch unterschiedliche Löslichkeiten oder verschiedene Stadien im Wachstum der Ausscheidungen erklären lassen, liegt der Schlüssel zum Verständnis der völlig verschiedenen Mikrostrukturen in der unterschiedlichen Volumenfehlpassung zwischen Metallsilizid und Si-Matrix.

\subsubsection{Volumenfehlpassung}

Die Volumenfehlpassung $\delta_{V}$ für Ausscheidungen eines Metallsilizids in Silizium ist nach Schröter et al. [27] definiert als 


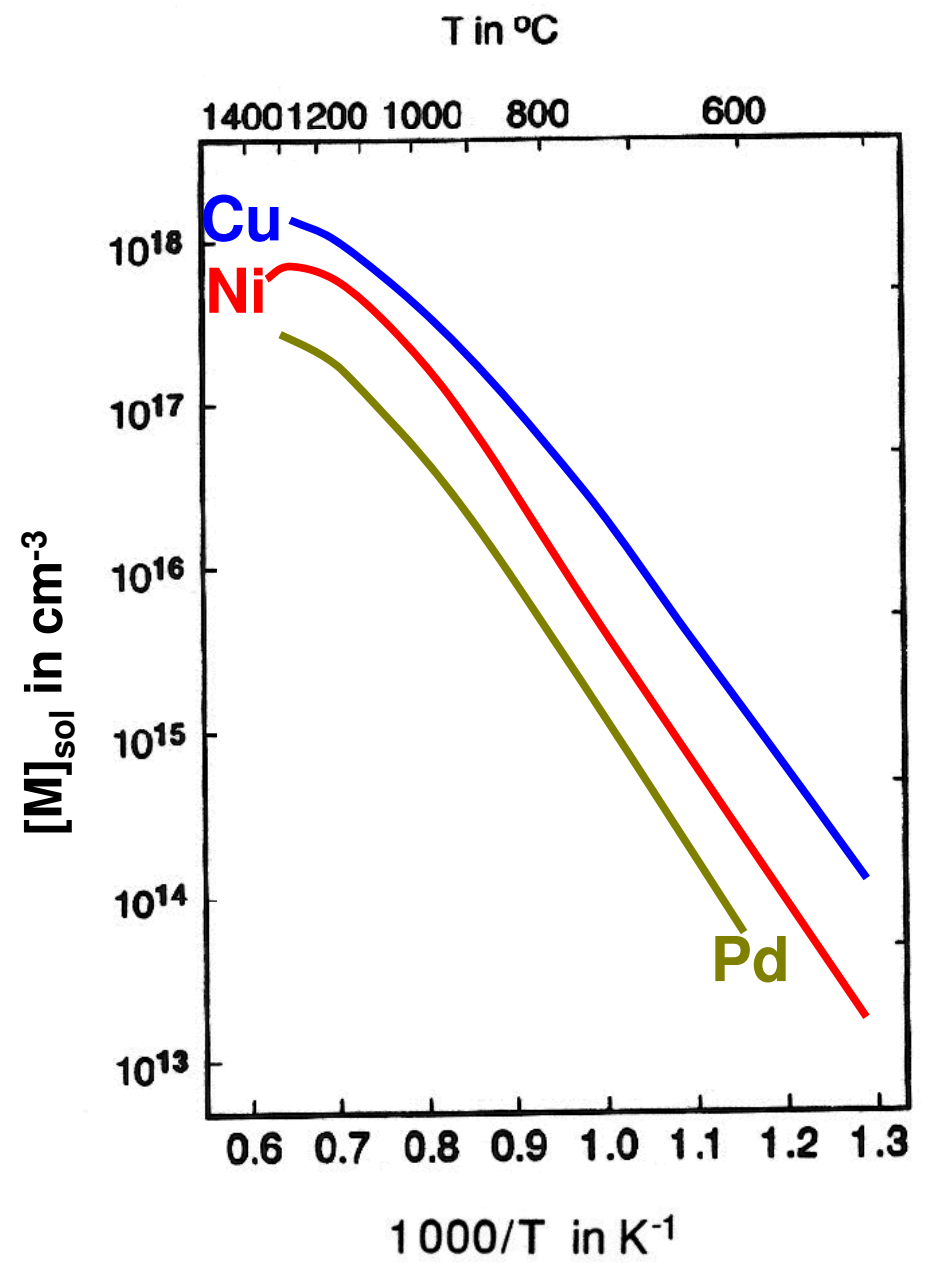

Abbildung 2.1. - Darstellung der Löslichkeit der Übergangselemente $\mathrm{Cu}, \mathrm{Ni}$ und $\mathrm{Pd}$ in Silizium als Funktion der inversen Temperatur nach [27] auf der Grundlage von Daten für $\mathrm{Cu}$ und $\mathrm{Ni}$ von Weber [39] und Daten für Pd von Frank [40].

$$
\delta_{V}=\frac{V_{S i l}-V_{S i}}{V_{S i}},
$$

wobei $V_{\text {Sil }}$ das Volumen der Silizidausscheidung und $V_{S i}$ das Volumen ist, das die in 
der Ausscheidung enthaltenen Si-Atome einnehmen, wenn sie in der Si-Matrix eingebaut sind. Die Volumenfehlpassung ist positiv bzw. negativ, wenn die Dichte der Si-Atome innerhalb des Silizids geringer bzw. höher ist als die Dichte der Gitterplätze in kristallinem Si. Bei positiver Volumenfehlpassung nimmt eine unverzerrte Ausscheidung des Silizids innerhalb der Matrix ein größeres Volumen ein als alle in der Ausscheidung enthaltenen Si-Atome zusammen. Bei kleinen Werten von $\delta_{V}$ und kleinen Ausscheidungen führt die Volumenfehlpassung zu elastischer Verzerrung, bei großen Werten oder großen Teilchen hingegen zu plastischer Verformung des Teilchens und der Matrix oder zur Erzeugung intrinsischer Punktdefekte des Siliziums. Der letztgenannte Effekt lässt sich nach Marioton und Gösele [46] durch eine quasi-chemische Reaktion beschreiben

$$
x M_{i}+y S i+P_{N} \leftrightarrow P_{N+1}+x \alpha I+x \beta V .
$$

Dabei bezeichnet $P_{N}$ eine Ausscheidung des Silizids, die $N$ Formeleinheiten des Silizids $M_{x} S i_{y}$ enthält. $\alpha$ und $\beta$ sind die Anzahlen der emittierten interstiellen Si-Atome $(I)$ und Leerstellen $(V)$ im Si-Gitter pro metallischem Fremdatom. Bei Fehlen elastischer Verzerrung und vollständiger Kompensation der Volumenfehlpassung durch die erzeugten intrinsischen Punktdefekte lässt sich der Unterschied der Volumina einer Ausscheidung aus $N$ Formeleinheiten des Silizids $M_{x} S i_{y}$ und der in der Ausscheidung enthaltenen Si-Atome angeben

$$
V_{S i l}-V_{S i}=N x(\alpha-\beta) \Omega
$$

wobei $\Omega$ das Volumen pro Gitterplatz in Silizium ist. Die in der Ausscheidung enthaltenen Si-Atome nehmen das Volumen $V_{S i}=N y \Omega$ ein. Für die Volumenfehlpassung nach Gl. 2.3 ergibt sich so

$$
\delta_{V}=\frac{x}{y}(\alpha-\beta)
$$

Da die Gitterkonstante des Siliziums und die Gitterparameter des Metallsilizids von der Temperatur abhängen, ist die Volumenfehlpassung eine temperaturabhängige Größe. Die Änderung $\Delta V$ des Volumens eines Festkörpers bei Änderung $\Delta T$ der Temperatur lässt sich durch den thermischen Ausdehnungskoeffizienten $\kappa$ beschreiben [47]:

$$
\Delta V=V-V_{0}=3 \kappa V_{0} \Delta T
$$

wobei $V_{0}$ das Volumen des Festkörpers bei $\Delta T=0$ ist. Durch Umstellung nach $V$ lassen sich das Volumen der Ausscheidung und der darin enthaltenen Si-Atome und 
somit auch die Volumenfehlpassung in Abhängigkeit von der Temperatur $T$ angeben:

$$
\delta_{V}(T)=\left(\delta_{V, 0}+1\right) \frac{1+3 \kappa_{S i l}\left(T-T_{0}\right)}{1+3 \kappa_{S i}\left(T-T_{0}\right)}-1
$$

Dabei ist $\delta_{V, 0}$ die Volumenfehlpassung bei der Temperatur $T_{0}$ und $\kappa_{S i l}$ und $\kappa_{S i}$ sind die thermischen Ausdehnungskoeffizienten des Metallsilizids bzw. des Siliziums.

\subsubsection{Nickel}

Die Ausscheidungen bestehen aus $\mathrm{NiSi}_{2}$ [27], das die kubische $\mathrm{CaF}_{2}$-Struktur und eine Gitterkonstante von $0.541 \mathrm{~nm}$ hat [48]. Es ergibt sich so nur eine sehr geringe relative Volumenfehlpassung zum Silizium von $-1.1 \%$ bei Raumtemperatur, $T=300 \mathrm{~K}$. Aufgrund unterschiedlicher thermischer Ausdehnungskoeffizienten von $\mathrm{NiSi}_{2}$ und $\mathrm{Si}$, $\kappa_{N i S i_{2}}=1.2 \cdot 10^{-5} / \mathrm{K}$ und $\kappa_{S i}=3 \cdot 10^{-6} / \mathrm{K}$ nach Murarka [48], besitzt $\mathrm{NiSi}_{2}$ bei $T>400^{\circ} \mathrm{C}$ eine größere Gitterkonstante als Si und die Volumenfehlpassung ändert ihr Vorzeichen, wie in Abb. 2.2 dargestellt ist.

Ausscheidungen der $\mathrm{NiSi}_{2}$-Phase wachsen infolge der im gesamten dargestellten Temperaturbereich geringen Volumenfehlpassung, ohne dass es zu erheblicher Gitterverzerrung kommt. Die Ausscheidungen haben die Form dicker Plättchen oder Polyeder, wobei die Grenzflächen zwischen Ausscheidung und Matrix parallel zu \{111\}-Ebenen liegen. Die Ausscheidungen werden bis zu einigen $\mu \mathrm{m}$ groß. [25]. Bei Ausscheidungen, die diese Größe erreichen, führt auch die geringe relative Volumenfehlpassung des $\mathrm{NiSi}_{2}$ zu einer signifikanten Gitterverzerrung, die durch Erzeugung von Versetzungen wieder relaxiert wird [25].

Die Keimbildung von $\mathrm{NiSi}_{2}$-Ausscheidungen findet in defektfreiem Silizium homogen [26] und bei Vorliegen kristallographischer Defekte wie beispielsweise Stapelfehlern heterogen statt [49]. Nach schneller Abkühlung $(\dot{T} \approx-1000 \mathrm{~K} / \mathrm{s})$ liegen metastabile Ausscheidungen in Form dünner Plättchen auf $\{111\}$-Ebenen vor, die lediglich aus zwei Atomlagen $\mathrm{NiSi}_{2}$ bestehen, wie Seibt et al. [50, 51] mit hochauflösender Transmissionselektronenmikroskopie zeigten. Bei Temperaturen unterhalb der Diffusionstemperatur kommt es durch Ostwaldreifung zu einem Dickenwachstum der Plättchen bei gleichzeitiger Verringerung der Anzahl, so dass sich schließlich die nach langsamer Abkühlung beobachtete Morphologie ergibt.

Für $\mathrm{NiSi}_{2}$-Ausscheidungen werden zwei Orientierungszusammenhänge beobachtet 50 , 51], die gemäß der üblichen Notation aus der Forschung an Oberflächensiliziden als Typ A und Typ B bezeichnet werden [52]. Für den Typ A werden alle kristallographischen Richtungen des $\mathrm{Si}$ in $\mathrm{NiSi}_{2}$ fortgesetzt, während Typ B eine Zwillingsorientierung zeigt. 


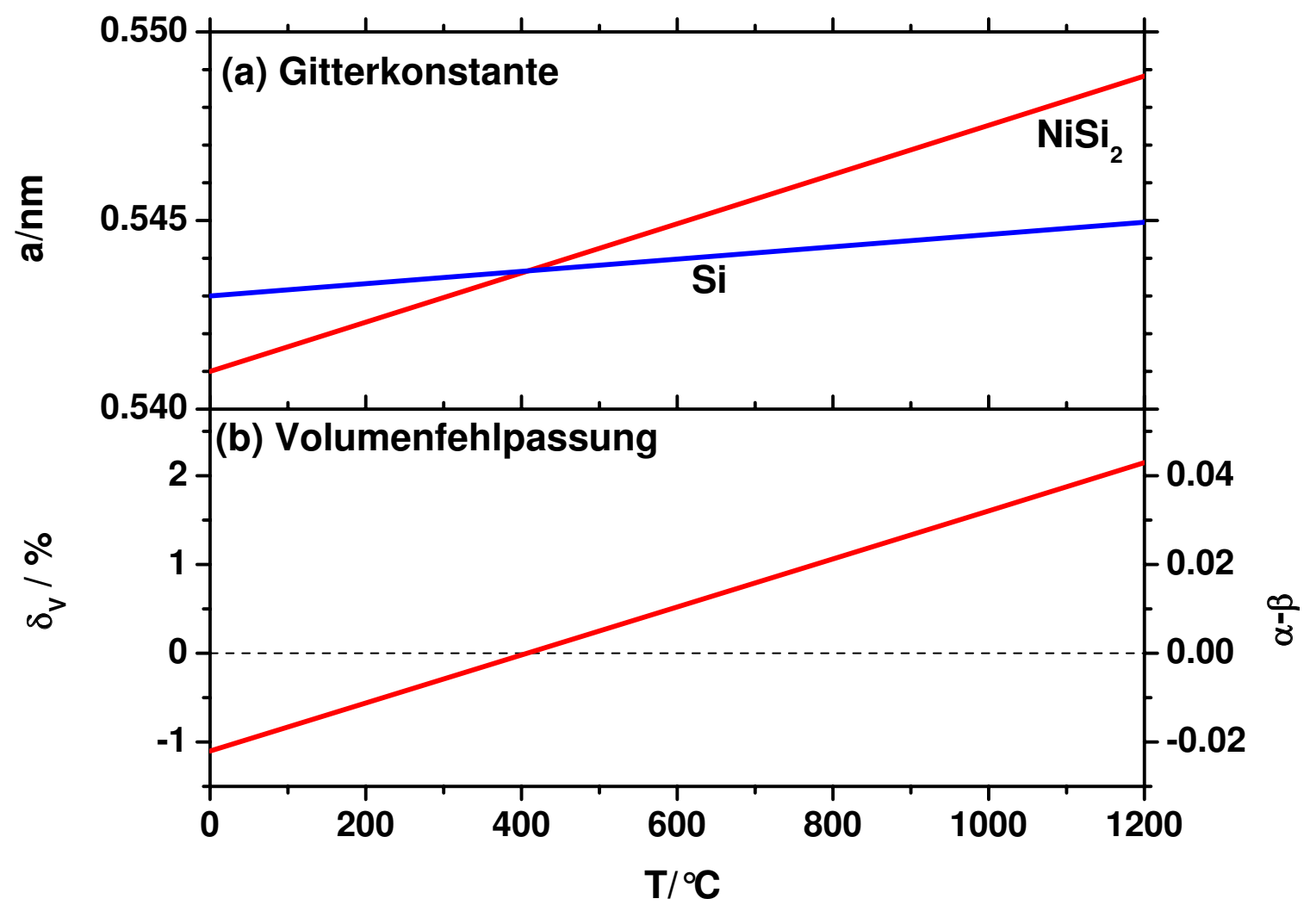

Abbildung 2.2. - (a) Abhängigkeit der Gitterkonstanten von $\mathrm{Si}$ und $\mathrm{NiSi}_{2}$ und (b) der resultierenden Volumenfehlpassung $\delta_{V}$ zwischen $\mathrm{Si}$ und $\mathrm{NiSi}_{2}$ von der Temperatur, nach Murarka [48]. Für den Fall vollständiger Kompensation der Volumenfehlpassung durch Erzeugung interstieller Si-Atome gibt der Wert $\alpha-\beta$ die Anzahl der pro ausgeschiedenem metallischen Fremdatom emittierten interstiellen Si-Atome an.

Hierbei sind die kristallographischen Richtungen des $\mathrm{Si}$ in $\mathrm{NiSi}_{2}$ um $180^{\circ}$ um eine Achse gedreht. Typ-A-Ausscheidungen liegen in Form von Plättchen oder Polyedern vor, wohingegen Typ-B-Ausscheidungen nur in plättchenförmiger Morphologie beobachtet wurden.

\subsubsection{Kupfer und Palladium}

Ausscheidungen von $\mathrm{Cu}$ oder $\mathrm{Pd}$ in $\mathrm{Si}$ bestehen anders als die Ausscheidungen von $\mathrm{Fe}$, $\mathrm{Co}$ oder Ni nicht aus silizumreichen sondern aus metallreichen Metallsiliziden [27]. Im Fall von Kupfer bestehen die Ausscheidungen aus der $\mathrm{Cu}_{3}$ Si-Phase, die im Phasendiagramm $\mathrm{Cu}-\mathrm{Si}$ in Abhängigkeit von der Temperatur verschiedene Modifikationen zeigt [53]. Ausscheidungen dieser Phase lässt sich eine hexagonale Struktur mit den Gitterparametern $a=0.708 \mathrm{~nm}$ und $c=0.738 \mathrm{~nm}$ zuordnen [44]. Ausscheidungen von Palladium bestehen 
aus der $\mathrm{Pd}_{2} \mathrm{Si}$-Phase, die eine hexagonale Struktur vom $\mathrm{Fe}_{2} \mathrm{P}-\mathrm{Typ}$ mit den Gitterparametern $a=0.649 \mathrm{~nm}$ und $c=0.343 \mathrm{~nm}$ hat [54]. Die Ausscheidungen weisen eine hohe relative Volumenfehlpassung gegenüber Silizium von $150 \%$ für $\mathrm{Cu}_{3} \mathrm{Si}$ und von $110 \%$ für $\mathrm{Pd}_{2} \mathrm{Si}$ auf, die das System vorwiegend durch die Erzeugung interstitieller Si-Atome kompensiert. Nach Gl. (2.6) entspricht die Volumenfehlpassung von $\mathrm{Cu}_{3} \mathrm{Si}$ und $\mathrm{Pd}_{2} \mathrm{Si}$ einer Emission von 0.5 bzw. 0.55 interstiellen Si-Atomen pro ausgeschiedenem metallischen Fremdatom, was um mehr als eine Größenordnung über dem Wert für $\mathrm{NiSi}_{2}$ liegt, vgl. Abb. 2.2. Der Einfluss unterschiedlicher thermischer Ausdehnungskoeffizienten auf die Volumenfehlpassung von $\mathrm{Cu}_{3} \mathrm{Si}$ und $\mathrm{Pd}_{2}$ Si ist also vernachlässigbar, wenn man annimmt, dass die Änderung der Gitterparameter von $\mathrm{Cu}_{3} \mathrm{Si}$ und $\mathrm{Pd}_{2} \mathrm{Si}$ infolge von Temperaturänderung von vergleichbarer Größenordnung wie bei $\mathrm{NiSi}_{2}$ ist.

Kupfer [14, 25, 42-44] und Palladium [25] scheiden sich in Si in Form sternförmiger Kolonien aus. Die Kolonien stellen planare Anordnungen einzelner kleiner Ausscheidungen auf $\{110\}$-Ebenen $(\mathrm{Cu})$ oder $\{111\}$-Ebenen $(\mathrm{Pd})$ dar und sind von Versetzungen berandet.

In Anlehnung an eine Arbeit von Silcock und Tunstall über das Wachstum von NbCAusscheidungen in austenitischem Stahl [55] haben Nes et al. [42, 56] und Solberg et al. [43] für den Wachstumsmechanismus der Kolonien einen autokatalytischen Prozess vorgeschlagen, der auf der Wechselwirkung von Ausscheidungen, die beim Wachstum interstitielle Si-Atome emittieren, und Versetzungen beruht, vgl. Abb. 2.3. Nach diesem Modell bilden sich zuerst an einer bestehenden Versetzungen durch heterogene Keimbildung kleine Ausscheidungen (Abb. 2.3a). Im nächsten Schritt wachsen die Ausscheidungen, wobei sie interstitielle Si-Atome erzeugen, die von der Versetzung absorbiert werden. Infolgedessen klettert die Versetzung und entfernt sich von der ursprünglichen Position (Abb. 2.3b). Die Ausscheidungen wandern mit der Versetzung. Im letzten Schritt löst

die Versetzung sich von den Ausscheidungen und stellt erneut einen Keimbildungsplatz für weitere Ausscheidungen dar (Abb. 2.3c).

\subsection{Ternäre Phasen aus Si und zwei Metallen}

Die Phasen eines ternären Systems lassen sich im ternären Phasendiagramm darstellen. Isothermische Schnitte durch das Phasendiagramm zeigen dabei die Existenzbereiche der ternären Phasen in Abhängigkeit von der Zusammensetzung des Systems, die gegeben ist durch die Molenbrüche der drei Komponenten $X_{1}, X_{2}$ und $X_{3}$, wobei $X_{1}+X_{2}+X_{3}=1$ gilt [57].

Setton gibt einen Überblick über Phasen, die bei Reaktionen zwischen MetallsilizidSchichten in ternären Systemen aus Si und zwei Metallen $M 1$ und $M 2$ gebildet werden [58]. Nach seinem Klassifikationsschema sind im Gleichgewicht mit Si zwei Arten von 


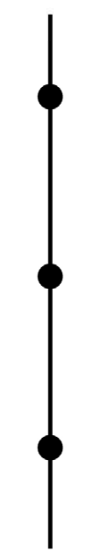

(a)

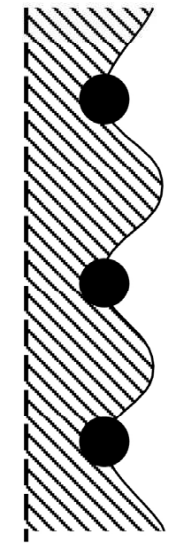

(b)

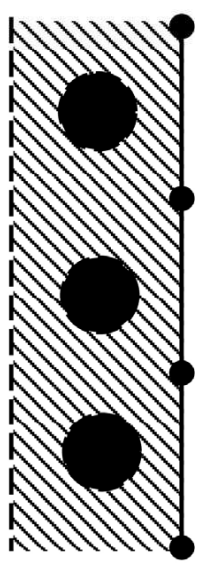

(c)

Abbildung 2.3. - Modell des Wachstums von Ausscheidungen an Versetzungen nach Solberg und Nes [43]: (a) heterogene Keimbildung von $\mathrm{Cu}_{3} \mathrm{Si}$-Ausscheidungen an einer Versetzung, (b): Wachstum der Ausscheidungen. Die Versetzung absorbiert durch Klettern interstitielle Si-Atome und entfernt sich von ihrer ursprünglichen durch die unterbrochen gezeichnete Linie markierten Position, (c) Ablösung der Versetzung von den Ausscheidungen und erneute Keimbildung

Phasen möglich:

1. Es kann ein binäres Silizid $M 1_{x} S i_{y}$ vorliegen, in dem zusätzlich die zweite metallische Verunreinigung in gelöster Form enthalten ist.

2. Es kann ein ternäres Silizid $M 1_{x} M 2_{y} S i_{z}$ auftreten, das sich im Gegensatz zu 1. nicht durch Mischen des zweiten Metalls in einem Silizid des ersten Metalls erhalten lässt.

Die Bildung ternärer Silizide ist um so wahrscheinlicher, je stärker sich $M 1$ und $M 2$ in ihren Eigenschaften (Schmelzpunkt, Anzahl der Valenzelektronen, Atomradius) unterscheiden, [59]. Ein Beispiel ist die Verbindung $\mathrm{Zr}_{2} \mathrm{CuSi}_{4}$ im System Cu-Si-Zr [60]. 
Haben dagegen die beiden Metalle ähnliche Eigenschaften, lässt sich eine hohe gegenseitige Löslichkeit der Metalle im Silizid des jeweils anderen Metalls beobachten, wie beispielsweise für Fe, das eine Löslichkeit von $30 \mathrm{At. \%}$ in $\mathrm{NiSi}_{2}$ zeigt [61]. Im Fall des Systems Co-Ni-Si gehen Finstadt et al. sogar von vollständiger Mischbarkeit von $\mathrm{CoSi}_{2}$ und $\mathrm{NiSi}_{2}$ aus [62]. Im Folgenden sollen die für diese Arbeit wichtigen Systeme Cu-Ni-Si und Ni-Pd-Si näher betrachtet werden.

\subsection{1. $\mathrm{Cu}-\mathrm{Ni}-\mathrm{Si}$}

Okamoto untersuchte die Erstarrung von Cu-Ni-Si-Flüssigkeiten und findet einen ternären eutektischen Punkt bei $769^{\circ} \mathrm{C}$ und einer Zusammensetzung von 56 At.\% Cu, 11 At.\% Ni und 33 At.\% Si, an dem die ternäre Flüssigkeit mit der Si-Phase, der $\mathrm{NiSi}_{2}$-Phase und einer ternären Silizid-Phase im Gleichgewicht steht [63]. In zusammenfassenden Darstellungen, die auf Okamoto verweisen, wird diese Phase unterschiedlich bezeichnet, bei Jänecke als $\omega$-Phase [64] und in der neuesten Darstellung des ternären Phasendiagramms als $\tau$-Phase [65].

Sokolovskaya et al. untersuchten Legierungen des Systems Cu-Ni-Si und geben einen isothermischen Schnitt bei $500^{\circ} \mathrm{C}$ durch das ternäre Phasendiagramm an [34]. Die nach diesem Schnitt bei $500^{\circ} \mathrm{C}$ koexistierenden Phasen in der silizumreichen Ecke des Systems $\mathrm{Cu}-\mathrm{Ni}$-Si sind in Abb. 2.4 dargestellt. Bei $500^{\circ} \mathrm{C}$ steht $\mathrm{Si}$ mit einer Lösung von $\mathrm{Cu}$ in $\mathrm{NiSi}_{2}$ (in dieser Arbeit als $\mathrm{NiSi}_{2}$ :Cu bezeichnet) und einer Lösung von $\mathrm{Ni}$ in $\mathrm{Cu}_{3} \mathrm{Si}$ (in dieser Arbeit als $\mathrm{Cu}_{3} \mathrm{Si}$ :Ni bezeichnet) im Gleichgewicht. Sokolovskaya et al. interpretieren dabei das ternäre Silizid, auf das Okamoto hinweist [63], als eine Modifikation der $\mathrm{Cu}_{3} \mathrm{Si}$-Phase, die bei höherer Temperatur durch den Einbau von Nickel stabilisiert wird. Die Löslichkeit von $\mathrm{Ni}$ in $\mathrm{Cu}_{3} \mathrm{Si}$ beträgt $5 \mathrm{At} . \% \mathrm{Ni}$ bei $500^{\circ} \mathrm{C}$, während sich für die Löslichkeit von $\mathrm{Cu}$ in $\mathrm{NiSi}_{2}$ ein Wert von etwa $1 \mathrm{At} . \%$ ablesen lässt.

\subsection{2. $\mathrm{Ni}-\mathrm{Pd}-\mathrm{Si}$}

Wopersnow und Schubert [66] finden im System Ni-Pd-Si bei $800^{\circ} \mathrm{C}$ eine ternäre MonosilizidPhase der Zusammensetzung $\mathrm{Ni}_{x} \mathrm{Pd}_{1-x} \mathrm{Si}$, die aus Mischung der PdSi- und NiSi-Phase hervor geht, welche jeweils die orthorhombische MnP-Struktur haben. Bei der Untersuchung der Reaktion dünner Ni- und $\mathrm{Pd}-\mathrm{Schichten}$ auf Si bei $800^{\circ} \mathrm{C}$ fanden Finstad und Nicolet [67] eine ternäre Phase der Zusammensetzung $\mathrm{Ni}_{0.65} \mathrm{Pd}_{0.35} \mathrm{Si}$ bei $800^{\circ} \mathrm{C}$, die sich ebenfalls der oben beschriebenen Monosilizidphase [66] zuordnen lässt.

Im System Ni-Pd-Si weist die Nickeldisilizidphase eine geringe Löslichkeit für Palladium auf. Sie wird daher in dieser Arbeit mit $\mathrm{NiSi}_{2}: \mathrm{Pd}$ bezeichnet. Loomans et al. untersuchten das Gleichgewicht zwischen Silizium, $\mathrm{NiSi}_{2}: \mathrm{Pd}$ und der ternären Monosilizidphase bei Temperaturen zwischen $760^{\circ} \mathrm{C}$ und $880^{\circ} \mathrm{C}$ in siliziumreichen Legierungen [68]. Sie messen bei $800^{\circ} \mathrm{C}$ für die Löslichkeit von $\mathrm{Pd}$ in $\mathrm{NiSi}_{2}$ einen Wert von 1.2 At.\% und geben für die Zusammensetzung der ternären Monosilizidphase $\mathrm{Ni}_{x} \mathrm{Pd}_{1-x} \mathrm{Si}$ einen Wert 


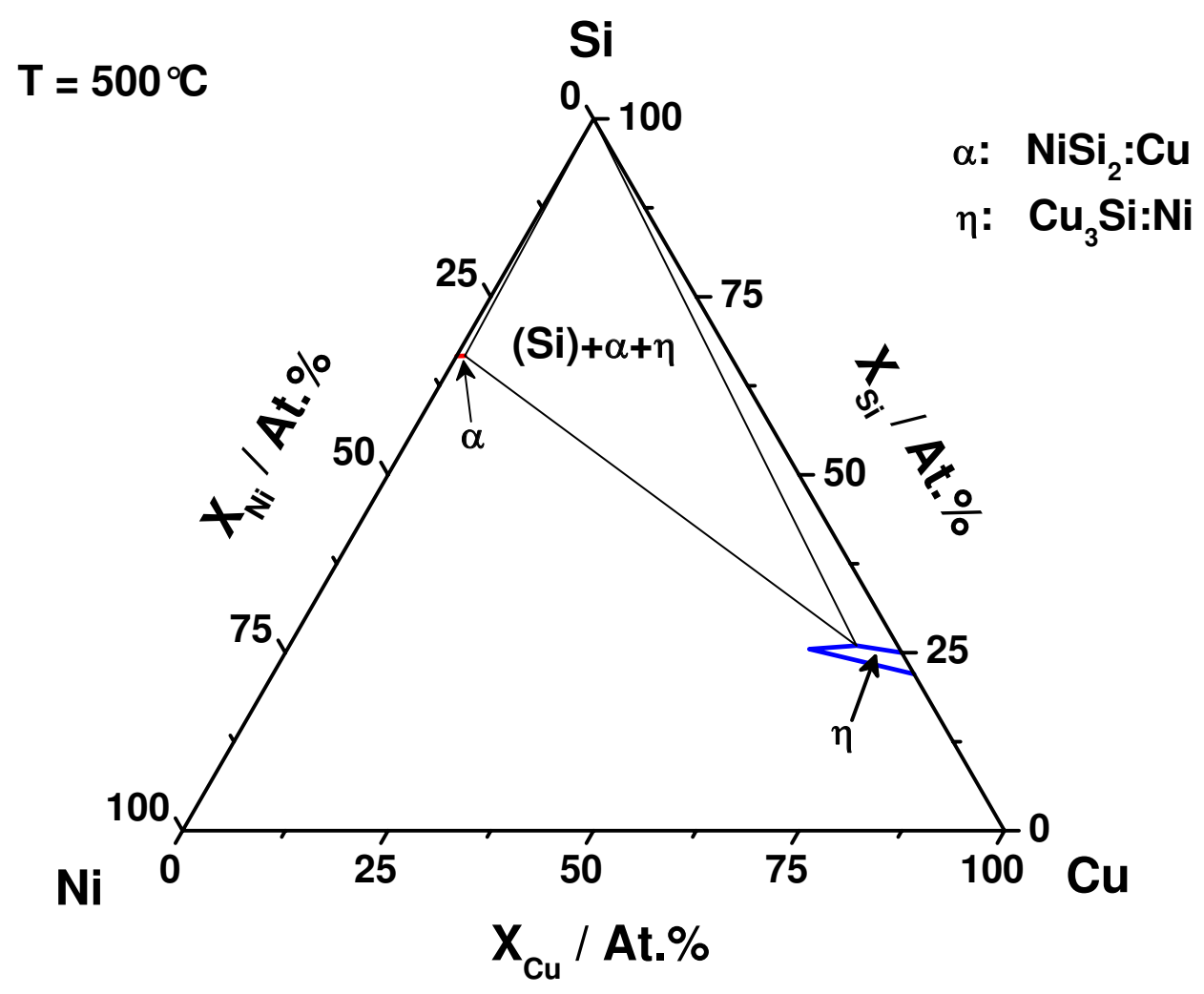

Abbildung 2.4. - Ausschnitt des isothermischen Schnittes bei $T=500^{\circ} \mathrm{C}$ durch das ternäre Phasendiagramm von $\mathrm{Cu}-\mathrm{Ni}$-Si nach Sokolovskaya et al. [34]. Dargestellt sind die Existenzbereiche der $\mathrm{NiSi}_{2}$ :Cu-Phase (einer Lösung von $\mathrm{Cu}$ in $\mathrm{NiSi}_{2}$ ), der $\mathrm{Cu}_{3} \mathrm{Si}: \mathrm{Ni}$ Phase (einer Lösung von $\mathrm{Ni}$ in $\mathrm{Cu}_{3} \mathrm{Si}$ ) und deren gemeinsamer Koexistenzbereich mit der Si-Phase, in der $\mathrm{Cu}$ und Ni gelöst sind.

von $\mathrm{x}=0.91$ an, was einem Pd-Anteil von 4.7 At.\% entspricht.

In der neuesten Veröffentlichung des ternären Phasendiagramms Pd-Ni-Si wird die Existenz der ternären Silizidphase $\mathrm{Ni}_{x} \mathrm{Pd}_{1-x} \mathrm{Si}$ in Legierungen mit einem hohen $\mathrm{Pd}-$ Anteil bei $800^{\circ} \mathrm{C}$ in Frage gestellt, da im binären System Pd-Si bei $800^{\circ} \mathrm{C}$ nicht PdSi sondern $\mathrm{Pd}_{2} \mathrm{Si}$ mit Si im Gleichgewicht steht [69].

\subsection{Koausscheidung von Übergangsmetallen}

Bei gleichzeitiger Verunreinigung von $\mathrm{Si}$ mit $\mathrm{Cu}$ und Fe lassen sich stabförmige, bei Verunreinigung mit $\mathrm{Cu}$ und $\mathrm{Ni}$ plättchenförmige Ausscheidungen finden, die jeweils von Kolonien kleinerer Ausscheidungen dekoriert werden [30-32]. Graff [26] deutet die Be- 
obachtungen von Cullis und Katz [30] als heterogene Keimbildung von Ausscheidungen aus $\mathrm{FeSi}_{2}$ an Kupfersilizidausscheidungen. Ryoo et al. [31] interpretieren die Teilchen in den Kolonien als Koausscheidungen von $\mathrm{Cu}$ und $\mathrm{Ni}$ in Form einer $(\mathrm{Cu}, \mathrm{Ni})-\mathrm{Si}_{2}$-Phase.

In jüngerer Vergangenheit haben Buonassisi et al. [28, 29, 70] an gezielt mit $\mathrm{Fe}, \mathrm{Cu}$ und Ni verunreinigtem ein- und multikristallinem Silizium [71] Untersuchungen zur Koausscheidung von Übergangsmetallen in Si durchgeführt.

In Proben die nach der Eindiffusion der Metalle bei $1200^{\circ} \mathrm{C}$ zuerst abgeschreckt und dann bei $655^{\circ} \mathrm{C}$ ausgelagert wurden, fanden sie mit Röntgenfluoreszenzmikroskopie und Röntgenabsorptionsspektroskopie [72] Anhäufungen von $\mathrm{Cu}$ und $\mathrm{Ni}$ an denselben Stellen, die sie als Kolonien kleiner Ausscheidungen entweder aus $\mathrm{Cu}_{3} \mathrm{Si}$ oder $\mathrm{NiSi}_{2}$ interpretieren [29]. Die Ausscheidungen sind lokal miteinander korreliert, weisen aber keine Phasen auf, in denen $\mathrm{Cu}$ und $\mathrm{Ni}$ gemischt sind. Nach Ansicht von Buonassisi et al. lassen sich diese Beobachtungen mit den herkömmlichen Modellen für Bildung und Wachstum von Metallsilizidausscheidungen in Si erklären, da sich die bekannten thermodynamischen Gleichgewichtsphasen $\mathrm{Cu}_{3} \mathrm{Si}$ und $\mathrm{NiSi}_{2}$ und die für $\mathrm{Cu}_{3} \mathrm{Si}$-Ausscheidungen typischen Kolonien bilden, vgl. Abschnitt 2.1.2. Die enge räumliche Korrelation von Ausscheidungen führen sie auf eine Begünstigung der Bildung und des Wachstums der Ausscheidungen durch eine Verringerung der Gitterverzerrung zurück, wenn sich Phasen mit Volumenfehlpassungen unterschiedlichen Vorzeichens (150\% für $\mathrm{Cu}_{3} \mathrm{Si}$ und $-1.1 \%$ für $\mathrm{NiSi}_{2}$ bei Raumtemperatur) am selben Ort bilden.

In Proben, die nach Eindiffusion der metallischen Verunreinigungen bei $1200^{\circ} \mathrm{C}$ langsam abgekühlt wurden, finden Buonassisi et al. [29] und Heuer et al. [28, 70] große Ausscheidungen, die in einer geringen Dichte vorliegen. Die Ausscheidungen sind dabei nicht homogen, sondern bestehen aus zwei separierten Phasen. Bei einer Phase handelt es sich um reines $\mathrm{Cu}_{3} \mathrm{Si}$ während in der zweiten Phase $\mathrm{Cu}, \mathrm{Ni}, \mathrm{Fe}$ und $\mathrm{Si}$ gemischt sind. Heuer et al. können experimentelle Röntgenabsorptionsspektren mit simulierten Spektren in Übereinstimmung bringen, die sich aus einem Modell für die Struktur der Phase ergeben [28]. Nach diesem Modell hat die zweite Phase eine $\mathrm{NiSi}_{2}$-Struktur, wobei die $\mathrm{Cu}$-Atome substitutionell auf Si-Plätzen und die Fe-Atome substitutionell auf Ni-Plätzen eingebaut sind.

Buonassisi et al. [29] schlagen für die Bildung der Ausscheidungen mit separaten Phasen einen neuen Mechanismus vor. Danach findet bei der Abkühlung noch oberhalb der eutektischen Temperatur die Bildung einer flüssigen Si-Cu Phase statt, in der sich die übrigen metallischen Verunreinigungen anreichern. Nach Durchlaufen der eutektischen Temperatur erstarrt die flüssige Phase, wobei es zur Separation der Cu-Ni-Fe-Si Mischphase und der $\mathrm{Cu}_{3} \mathrm{Si}$ Phase kommt. 
Kapitel 2

\section{Methoden}

\subsection{Transmissionselektronenmikroskopie (TEM)}

In der vorliegenden Arbeit wird die Transmissionselektronenmikroskopie (TEM) zur Untersuchung der strukturellen und chemischen Eigenschaften von Metallsilizidausscheidungen eingesetzt. Neben der konventionellen Abbildung zum Nachweis der Ausscheidung, etwaiger Sekundäreffekte und Verzerrungsfelder wurden die hochauflösende Elektronenmikroskopie (HRTEM) und die Elektronenbeugung herangezogen, um kristallographische Informationen zu bekommen. Die eingesetzten Standardverfahren sind in der Literatur [73-76] eingehend beschrieben, so dass hier auf eine Darstellung verzichtet werden kann.

Das verwendete Mikroskop ist ein CM200-UT FEG der Firma Philips mit einer Beschleunigungsspannung von $200 \mathrm{keV}$ und einer Feldemissionskathode als Elektronenquelle.

\subsection{Energiedispersive Röntgenspektroskopie (EDX)}

Ein wichtiges Werkzeug dieser Arbeit für die Untersuchung der Zusammensetzung der Metallsilizidausscheidungen ist die energiedispersive Röntgenspektroskopie (EDX). Sie besteht in der Detektion und Analyse von Röntgenstrahlung, die als Folge der Wechselwirkungen zwischen Probe und Elektronenstrahl erzeugt wird. In der Probe entsteht Bremsstrahlung und charakteristische Strahlung. Das Spektrum der charakteristischen Strahlung erlaubt einen Rückschluss auf die enthaltenen Elemente. Sie besteht aus Röntgenquanten, deren Energie dem Übergang eines Elektrons von einer äußeren auf eine innere Schale eines Atoms der Probe entspricht. Zur Aussendung dieser Quanten kommt es, wenn Atome, die zuvor durch Wechselwirkung mit dem Elektronenstrahl in einen $\mathrm{Zu}-$ stand höherer Energie angeregt worden sind, wieder in ihren Grundzustand relaxieren. 
Das Attribut „energiedispersiv“ bezeichnet die Art der Detektion der Röntgenquanten. Anders als im Fall wellenlängendispersiver Spektroskopie (WDS) zeichnet bei EDX ein Spektrometer die Intensität der Röntgenstrahlung in Abhängigkeit von der Energie der Röntgenquanten auf, [76]. Das Kernstück des Spektrometers ist ein Halbleiterdetektor, in dem ein Röntgenquant einen Strompuls erzeugt, dessen Höhe proportional zur Energie des Quants ist. Das gemessene Spektrum stellt im Prinzip eine Auftragung der Zahl der Pulse als Funktion der Pulshöhe dar.

Einen ausführlichen Einblick in die Methode und ihre Verwendung in Kombination mit TEM geben Williams und Carter, [76]. In der vorliegenden Arbeit kam ein Link ISIS System der Firma Oxford Instruments mit einem Si(Li)-Kristall als Detektor und einer Energieauflösung von $136 \mathrm{eV}$ zum Einsatz. Stolze schildert die praktische Benutzung dieses Systems in [77].



Abbildung 3.1. - EDX-Spektrum einer Ausscheidung, die aus $\mathrm{Cu}$, $\mathrm{Ni}$ und Si bestehen. Aufgetragen ist die Anzahl der Zählereignisse in Abhängigkeit von der Energie der Röntgenquanten. 
Abb. 3.1 zeigt ein EDX-Spektrum, das im Rahmen dieser Arbeit an einer Stelle aufgenommen wurde, an der sich eine Ausscheidung innerhalb der Si-Matrix befindet. Die Ausscheidung lässt sich aufgrund struktureller Merkmale der binären Phase Nickeldisilizid zuordnen. Das Vorliegen der charakteristischen Linien nicht nur von Silizium und Nickel sondern auch von Kupfer zeigt, dass die Ausscheidung tatsächlich dreikomponentig ist. Die Bezeichnung der Linien, $\mathrm{K}_{\alpha}, \mathrm{K}_{\beta}$ und $\mathrm{L}_{\alpha}$ ergibt sich aus der Nomenklatur der Elektronenschalen.

Ein Standardverfahren für die quantitative Bestimmung der Zusammensetzung der Probe aus einem EDX-Spektrum ist die Methode von Cliff und Lorimer, [78]. Der Zusammenhang von Zusammensetzung und Signalintensität lässt sich durch die sogenannte Cliff-Lorimer-Gleichung darstellen:

$$
\frac{C_{A}}{C_{B}}=k_{A B} \frac{I_{A}}{I_{B}}
$$

Darin bezeichnen $C_{A}$ und $C_{B}$ die Anteile der Elemente $A$ und $B$ an der Zusammensetzung und $I_{A}$ und $I_{B}$ die Intensitäten der für $A$ und $B$ charakteristischen Linien im gemessenen EDX-Spektrum. Die Zusammensetzungen werden relativ ausgedrückt, so dass $C_{A}+C_{B}=1$ ist.

In dünnen Proben, wie sie für TEM-Folien zumeist vorliegen, lassen sich Absorption und Fluoreszenz in der Probe vernachlässigen. Der Faktor $k_{A B}$ hängt in dünnen Proben noch von den Kernladungszahlungen $Z_{A}$ und $Z_{B}$ der Elemente, der Beschleunigungsspannung der Elektronen, dem EDX-Detektor und dem zur Filterung der Bremsstrahlung verwendeten Algorithmus ab. Für die Elemente $\mathrm{Cu}, \mathrm{Ni}, \mathrm{Pd}$ und $\mathrm{Si}$, deren Anteile an der Zusammensetzung von Ausscheidungen in dieser Arbeit bestimmt werden, und das genutzte EDX-TEM-System sind die $k$-Faktoren mit $\mathrm{Cu}$ als Referenzelement in Tab. 3.1 angegeben.

\begin{tabular}{|c|c|c|c|c|}
\hline Element A & $\mathrm{Cu}$ & $\mathrm{Ni}$ & $\mathrm{Pd}$ & $\mathrm{Si}$ \\
\hline$k_{A C u}$ & 1 & 0.911 & 1.257 & 0.712 \\
\hline Linie & $\mathrm{Cu}-\mathrm{K}$ & Ni-K & Pd-L & Si-K \\
\hline
\end{tabular}

Tabelle 3.1. - k-Faktoren und Linien der charakteristischen Röntgenstrahlung der in dieser Arbeit untersuchten Elemente $\mathrm{Cu}, \mathrm{Ni}, \mathrm{Pd}$ und Si für die quantitative Analyse von EDXSpektren nach Cliff und Lorimer [78]

Die Stärke der Kombination von EDX und TEM liegt darin, dass sich die chemische und strukturelle Informationen miteinander in Zusammenhang setzen lassen. Dazu wird das TEM im Rastermodus betrieben. Der Elektronenstrahl lässt sich bei dem in dieser Arbeit verwendeten Gerät bis auf einen Durchmesser von 1 bis $2 \mathrm{~nm}$ fokussieren, so dass sich die Zusammensetzung der Probe nach der Erfassung eines EDX-Spektrums 
punktuell mit hoher Ortsauflösung untersuchen lässt. Gilt es, die laterale Verteilung von Elementen in einem größeren Bereich der Probe zu untersuchen, so erweist es sich als hilfreich, die charakteristische Röntgenstrahlung zu messen, während der Elektronenstrahl die Probe abtastet. Dazu werden vor der Messung in der Umgebung der charakteristischen Linien Spektralbereiche definiert, innerhalb derer alle Zählereignisse zum Signal der jeweiligen Linie beitragen. Auf diese Weise lassen sich darstellen:

- Zusammensetzung entlang einer bestimmten Strecke

- zweidimensionale Verteilung verschiedener Elemente (EDX-Karte)

Es ist allerdings zu beachten, dass die so erhaltenen Daten die Beiträge der Bremsstrahlung enthalten. Diese werden im Fall der oben beschriebenen Auswertung nach Cliff und Lorimer durch numerische Anpassung aus der Analyse eliminiert. 


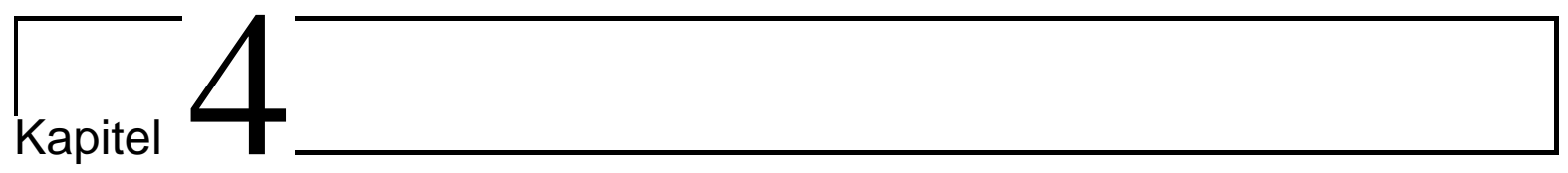

\section{Ergebnisse}

In dieser Arbeit wurden Ausscheidungen metallischer Verunreinigungen in Silizium untersucht, das vergleichbare Eigenschaften wie das in der Photovoltaik gegenwärtig intensiv eingesetzte multikristalline Silizium hat, welches Korngrenzen und Versetzungen und außerdem Ausscheidungen nichtmetallischer Verunreinigungen wie beispielsweise Sauerstoff enthält.

Das Probenmaterial sollte im Ausgangszustand strukturelle Defekte und Ausscheidungen nichtmetallischer Verunreinigungen, aber keine metallischen Verunreinigungen enthalten. Diese sollten vielmehr gezielt von außen durch Diffusion in das Material eingebracht und während langsamer Abkühlung durch heterogene Keimbildung an den im Material vorhandenen Defekten zur Ausscheidung gebracht werden.

Daher kam ein bikristallines Probenmaterial zum Einsatz, das durch Waferbonding aus zwei einkristallinen Scheiben Siliziumscheiben hergestellt wurde. Das Verfahren und die Beschaffenheit des Bikristall wird in Anhang A näher beschrieben. An der Grenzfläche der beiden miteinander verbundenen Siliziumscheiben besteht ein Netzwerk aus Stufenund Schraubenversetzungen, das einer Kleinwinkelkorngrenze entspricht. Der Abstand zwischen den Stufenversetzungen beträgt $372 \mathrm{~nm}$ und der zwischen den Schraubenversetzungen $12 \mathrm{~nm}$. Eine der beiden Siliziumscheiben enthält außerdem Mikrodefekte in einer Dichte von etwa $10^{10} \mathrm{~cm}^{-3}$, bei denen es sich Siliziumoxidausscheidungen und Versetzungsringe handelt.

\subsection{Ausscheidungen nach Diffusion von Kupfer}

Als erster Schritt wurde untersucht, wie sich Ausscheidungen einer einzigen metallischen Verunreinigung unter den Bedingungen miteinander konkurrierender Keimbildungsplätze bilden. Dazu werden Proben der Bikristalle zunächst durch Diffusion von Kupfer bei 
einer Temperatur von $T=1050^{\circ} \mathrm{C}$ gezielt verunreinigt und anschließend langsam mit $\dot{T}=-6 \mathrm{~K} / \mathrm{s}$ abgekühlt, so dass sich an den verschiedenen Keimbildungsplätzen Ausscheidungen bilden konnten. Einzelheiten der Präparation der Diffusionsproben befinden sich in Anhang B.1 (Proben 1 und 2 in Tab. B.1).



Abbildung 4.1. - Lichtmikroskopische Aufnahme eines Querschnitts durch eine bikristalline Probe aus Si nach Diffusion von $\mathrm{Cu}$ bei $1050^{\circ} \mathrm{C}$, langsamer Abkühlung und Defektätzung (Secco, 6s). Die Probe besteht aus zwei Scheiben (A und B). Die als Diffusionsquelle dienende Schicht befindet sich auf der der Oberfläche Of-1. Ein Stück defektfreien Siliziums (bezeichnet mit Ref) wurde vor dem Defektätzen auf Scheibe A zur Erkennung von Artefakten angebracht.

Abb. 4.1 und Abb. 4.2 zeigen lichtmikroskopische Aufnahmen zweier Querschnittsproben nach Defektätzen. Es ist bekannt, dass Kupfer als einzelne metallische Verunreinigung in Silizium Kolonien kleiner Ausscheidungen einer Kupfersilizidphase $\left(\mathrm{Cu}_{3} \mathrm{Si}\right)$ bildet. Defektätzen führt zur Entstehung linien- oder sternförmiger Ätzgrübchen am Ort der Kolonien [25, 42].

Die laterale Verteilung der Ätzgrübchen ist inhomogen und zeigt, dass die Dichte der Kupfersilizidausscheidungen in Scheibe B wesentlich höher ist als in Scheibe A. Diese 




Abbildung 4.2. - Lichtmikroskopische Aufnahme eines Querschnitts durch eine bikristalline Probe aus Si nach Diffusion von $\mathrm{Cu}$ bei $1050^{\circ} \mathrm{C}$, langsamer Abkühlung und Defektätzung (Secco, 6s). Die Probe besteht aus zwei Scheiben (A und B). Die als Diffusionsquelle dienende Schicht befindet sich hier auf der der Oberfläche Of-2. Ref: Ein Stück defektfreien Siliziums wurde vor dem Defektätzen auf Scheibe B zur Erkennung von Artefakten angebracht.

Beobachtung ist unabhängig von der Lage der als Quelle für die Diffusion verwendeten Schichten, wie der Vergleich der beiden in Abb. 4.1 und Abb. 4.2 dargestellten Proben deutlich macht. Die als Diffusionsquelle dienende Schicht befindet sich bei diesen Proben auf gegenüberliegenden Probenoberflächen, in Abb. 4.1 auf Oberfläche Of-1 und in Abb. 4.2 auf Oberfläche Of-2. Die inhomogene Verteilung kommt nicht durch eine Wirkung der Korngrenze als Diffusionsbarriere zustande, sondern lässt sich auf die Anwesenheit von Mikrodefekten in Scheibe B zurückführen, vgl. Abb. A.1 in Anhang A. Es handelt sich um kleine Siliziumoxidausscheidungen und Versetzungsringe, die die Oxidteilchen beim Wachstum ausgestoßen haben. Die Mikrodefekte stellen heterogene Keimbildungsplätze für Kupfersilizidausscheidungen dar.

Die Verteilung der Kupfersilizidausscheidungen in Scheibe B in Abb. 4.2 zeigt eine 
ausscheidungsfreie Zone einer Breite von ungefähr $200 \mu \mathrm{m}$ direkt unterhalb der Oberfläche Of-2. Die Beobachtung lässt sich durch die Nähe der als Diffusionsquelle verwendeten Schicht erklären. Diese Schicht bestand nach dem binären Phasendiagramm aus einer Flüssigkeit aus $\mathrm{Cu}$ und Si, da die Diffusionstemperatur $\mathrm{T}=1050^{\circ} \mathrm{C}$ oberhalb der Temperatur $T_{\text {eut }}=802^{\circ} \mathrm{C}$ des eutektischen Punktes zwischen Si und $\mathrm{Cu}_{3} \mathrm{Si}$ liegt, vgl. Abschnitt 2.1.1. Im Gleichgewicht mit der Flüssigkeit bzw. mit $\mathrm{Cu}_{3} \mathrm{Si}$ unterhalb der eutektischen Temperatur stellt sich an der Oberfläche Of-2 die Löslichkeit ein, so dass die metallischen Fremdatome während der langsamen Abkühlung aus dem oberflächennahen Bereich der Scheibe B in die Flüssigkeit heraus diffundierten, bevor sich Ausscheidungen bilden konnten.

In Scheibe A haben sich nur sehr wenige Kupfersilizidausscheidungen gebildet. Dies lässt sich auf eine sehr geringe Dichte als heterogenen Keimbildungszentren wirkender Mikrodefekte zurückführen.

Bei der in Abb. 4.1 untersuchten Probe liegt die Gleichgewichtsphase aus $\mathrm{Cu}$ und Si auf der Oberfläche Of-1 vor. Hier zeigt die Verteilung der Kupfersilizidausscheidungen keine ausscheidungsfreie Zone unterhalb der Oberfläche Of-1 in der Scheibe B keine ausscheidungsfreie Zone, während praktisch die gesamte Scheibe A ausscheidungsfrei ist.

An der Korngrenze bildeten sich ebenfalls Kupfersilizidausscheidungen. Es existiert keine ausscheidungsfreie Zone in der Nähe der Korngrenze in Scheibe B. 


\subsection{Ausscheidungen nach Kodiffusion von Kupfer und Nickel}

Unter denselben Bedingungen wie bei Kupfer als alleiniger Verunreinigung, d.h. bei $T=1050{ }^{\circ} \mathrm{C}, \dot{T}=-6 \mathrm{~K} / \mathrm{s}$ und in bikristallinem Probenmaterial wurden Ausscheidungen zweier metallischer Verunreinigungen durch gleichzeitige Diffusion, im folgenden als Kodiffusion bezeichnet, und anschließende langsame Abkühlung präpariert. Einzelheiten der Probenpräparation und eine Übersicht über die durchgeführten Diffusionsexperimente (Tabelle B.1) befinden sich in Anhang B.1.

Die Beschränkung auf zwei metallische Verunreinigungen ist sinnvoll, weil sich so Änderungen im Ausscheidungsverhalten der einen metallischen Verunreinigung gegenüber dem bekannten Verhalten bei Vorliegen als einziger Verunreinigung mit Eigenschaften der zweiten Verunreinigung in Beziehung setzen lassen.

Eine wichtige Eigenschaft metallischer Verunreinigungen ist die Volumenfehlpassung der Metallsilizidphase, aus der ihre Ausscheidungen bestehen. Zunächst wurden Kupfer und Nickel als metallische Verunreinigungen ausgewählt. Diese Verunreinigungen bilden in Si Ausscheidungen aus Metallsilizidphasen mit höchst unterschiedlicher Volumenfehlpassung, wodurch die Form, Größe und Verteilung ihrer Ausscheidungen bestimmt wird, wie in Kapitel 2.1.2 gezeigt wurde. Im Fall von Kupfer als alleiniger metallischer Verunreinigung mit hoher Volumenfehlpassung des Silizids $\left(\mathrm{Cu}_{3} \mathrm{Si}\right.$ : $\left.150 \%\right)$ bilden sich von Versetzungen berandete Kolonien kleinerer Teilchen und im Fall von Nickel mit geringer Volumenfehlpassung des Silizids $\left(\mathrm{NiSi}_{2}:-1.1 \%\right.$ bei Raumtemperatur) einzelne große Ausscheidungen.

Ein experimenteller Parameter der Kodiffusion zweier metallischer Verunreinigungen, der über die Löslichkeiten der Verunreinigungen in Silizium indirekt einstellbar ist, ist das Konzentrationsverhältnis $Q_{M_{1}, M_{2}}$ der Verunreinigungen:

$$
Q_{M_{1}, M_{2}}=\frac{\left[M_{1}\right]}{\left[M_{2}\right]}
$$

Löslichkeit und Diffusionskoeffizient von Kupfer, Nickel und Palladium, das in Kapitel 4.3 Kupfer als eine der beiden metallischen Verunreinigungen ersetzt, in Silizium bei der in dieser Arbeit vorwiegend benutzten Temperatur von $1050^{\circ} \mathrm{C}$ sind in Tabelle 4.1 angegeben.

\subsubsection{Ausscheidungen unter kupferreichen Bedingungen}

Nach Kodiffusion von Kupfer und Nickel liegen kupferreiche Bedingungen $Q_{C u, N i}>1$ vor, da die Löslichkeit von Kupfer höher ist als die von Nickel (Probe 3, Tab. B.1 in 


\begin{tabular}{c|cc|cl}
$\mathrm{M}$ & {$[M]_{\text {sol }}\left(\mathrm{cm}^{-3}\right)$} & Referenz & $D_{M}\left(\mathrm{~cm}^{2} \mathrm{~s}^{-1}\right)$ & Referenz \\
\hline $\mathrm{Cu}$ & $6 \cdot 10^{17}$ & Weber [39] & $2 \cdot 10^{-4}$ & Hall und Racette [36] \\
$\mathrm{Ni}$ & $3 \cdot 10^{17}$ & Weber [39] & $3 \cdot 10^{-5}$ & Bakhadyrkhanov et al. [37] \\
$\mathrm{Pd}$ & $1 \cdot 10^{17}$ & Frank [40] & $3 \cdot 10^{-5}$ & Graff et al. [38]
\end{tabular}

Tabelle 4.1. - Löslichkeit $[M]_{\text {sol }}$ und Diffusionskoeffizient $D_{M}$ von Kupfer, Nickel und Palladium bei $1050^{\circ} \mathrm{C}$ in Silizium

Anhang B.1). Physikalisch besteht unter kupferreichen Bedingungen eine Situation, in der zu erwarten ist, dass Form, Größe und Verteilung der Ausscheidungen durch hohe Volumenfehlpassung bestimmt ist.

\subsubsection{Verteilung}

Die Verteilung der Ausscheidungen nach Kodiffusion von Kupfer und Nickel unter kupferreichen Bedingungen im bikristallinen Silizium wurde nach Defektätzen lichtmikroskopisch untersucht. Abb. 4.3 zeigt das Ergebnis der Ätzgrübchenuntersuchung.

Die Verteilung der Ätzgrübchen in der Probe ist inhomogen und qualitativ dieselbe wie nach der Diffusion von Kupfer als alleiniger metallischer Verunreinigung, vgl. Abschnitt 4.1. Dies zeigt sich beim Vergleich von Abb. 4.3 mit Abb. 4.1 und Abb. 4.2 insbesondere am Fehlen einer defektfreien Zone an der Korngrenze in Scheibe B.

Die linien- und sternförmigen Ätzgrübchen entsprechen planaren Defekten, wobei linienförmige Ätzgrübchen auf einzelne dieser Defekte und sternförmige Ätzgrübchen auf Anordnungen mehrerer Defekte hinweisen. Diese Defekte bestehen aus Kolonien kleinerer Ausscheidungen, wie der folgende Abschnitt 4.2.1.2 zeigt.

Die Defekte liegen auf $\{110\}$-Ebenen, wie durch die Richtungsbeziehung der Ätzgrübchen mit der Korngrenze auf der Oberfläche der Querschnittsprobe in Abb. 4.3 deutlich wird. Durch die Präparation der Probe ist gewährleistet, dass die Ätzgrübchen auf einer $\{110\}$-Oberfläche der Probe liegen. Bei Festlegung dieser Oberfläche als (110)-Ebene ergeben sich für die Schnittlinien planarer Defekte auf $\{110\}$-Ebenen mit der Probenoberfläche die in Tab. 4.2 angegebenen Richtungsvektoren.

Da die Oberfläche der Querschnittsprobe eine $\{110\}$-Ebene ist, liegen Defekte auch parallel zur Probenoberfläche. Dies ist beispielsweise der Fall für den Defekt, der das in Abb. 4.3 mit P gekennzeichnete elliptisch-unregelmäßig geformte Ätzgrübchen verursacht hat. 


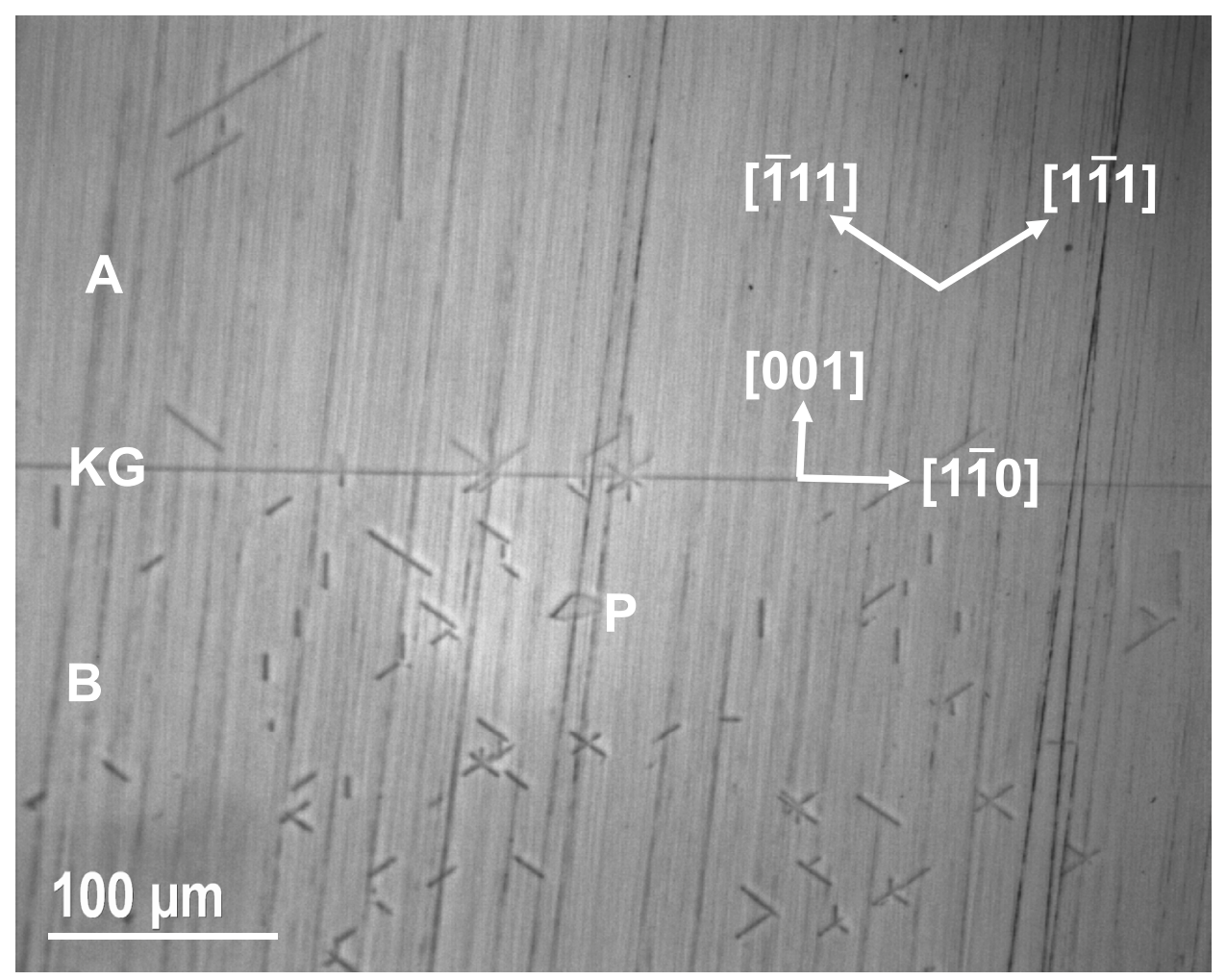

Abbildung 4.3. - Lichtmikroskopische Aufnahme des Querschnitts einer bikristallinen Probe nach Kodiffusion von $\mathrm{Cu}$ und $\mathrm{Ni}$ bei $1050^{\circ} \mathrm{C}$, langsamer Abkühlung und Defektätzung (Secco, 5s). Linienförmige Ätzgrübchen markieren zahlreiche Defekte an der Korngrenze $(K G)$ und im Inneren der beiden Scheiben $(A, B)$. Eines der Ätzgrübchen zeigt einen planaren Defekt $(P)$, der parallel zur Probenoberfläche liegt.

\begin{tabular}{|c|c|}
\hline Defekt & Schnittlinie \\
\hline$(110)$ & - \\
$(1 \overline{1} 0)$ & {$[001]$} \\
$(101)$ & {$[\overline{1} 11]$} \\
$(10 \overline{1})$ & {$[\overline{1} 1]$} \\
$(011)$ & {$[\overline{1} 1]$} \\
$(01 \overline{1})$ & {$[\overline{1} 11]$} \\
\hline
\end{tabular}

Tabelle 4.2. - Richtung der Schnittlinien planarer Defekte auf $\{110\}$-Ebenen mit der Oberfläche einer [110]-orientierten Probe. 


\subsubsection{Struktur}

Nach Kodiffusion von Kupfer und Nickel unter kupferreichen Bedingungen im bikristallinen Silizium lässt sich mit TEM beobachten, dass die planaren Defekte, deren Verteilung in der Probe im vorangegangenen Abschnitt 4.2.1.1 untersucht worden ist, aus Kolonien kleinerer Teilchen bestehen, wie Abb 4.4 zeigt. Die naheliegende Annahme, dass es sich bei diesen Teilchen um Ausscheidungen einer zweiten Phase (neben Silizium) handelte, wurde durch strukturelle und chemische Analysen bestätigt, deren Ergebnisse im Folgenden gezeigt werden.

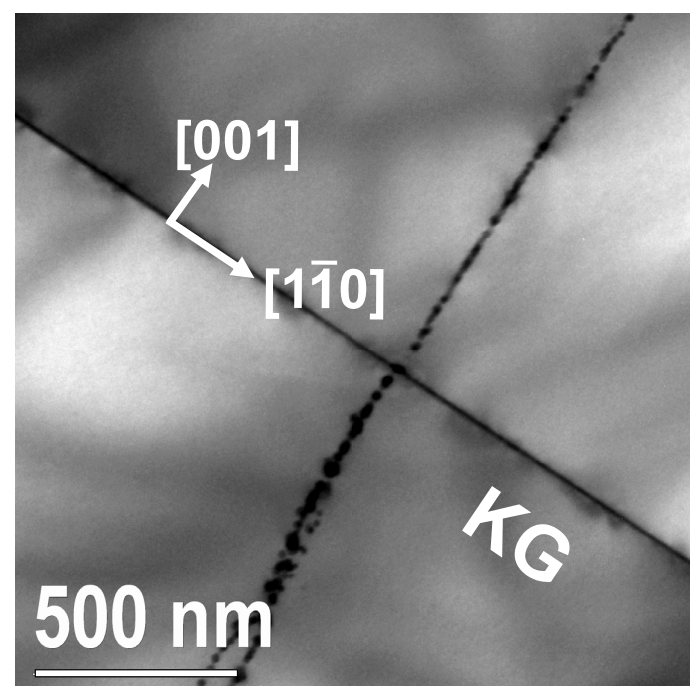

(a)

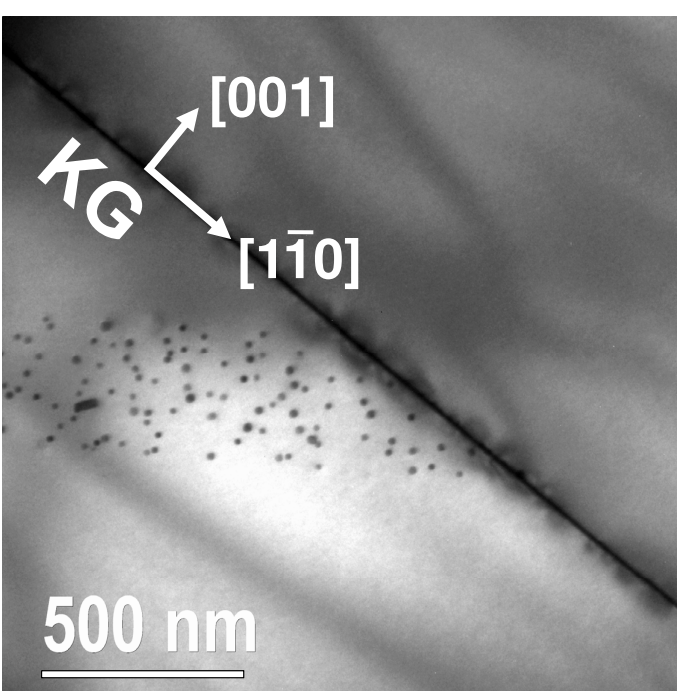

(b)

Abbildung 4.4. - TEM-Hellfeld-Aufnahmen zweier Kolonien kleiner Ausscheidungen an der Korngrenze (KG) einer bikristallinen Probe nach Kodiffusion von $\mathrm{Cu}$ und $\mathrm{Ni}$ bei $1050^{\circ} \mathrm{C}$ und langsamer Abkühlung. Die Ausscheidungen sind auf Ebenen angeordnet, die unterschiedlich orientiert sind: (a) senkrecht zur Bildebene und (b) gegenüber der Bildebene geneigt.

Die Kolonien bestehen aus planaren Anordnungen von Ausscheidungen. Die Ebenen, auf denen die Kolonien angesiedelt sind, haben unterschiedliche Orientierung, wie durch den Vergleich von Abb.4.4a und 4.4b deutlich wird, die die Probe in derselben Projektion (in [110]-Richtung) abbilden. Abb. 4.4 a zeigt eine Kolonie exakt von der Seite her, diese Kolonie verläuft somit senkrecht zur Bildebene. Dagegen zeigt Abb. 4.4b eine Kolonie auf einer gegenüber der Bildebene geneigten Ebene. Die scheinbaren seitlichen Begrenzungen dieser Kolonie sind keine tatsächlichen Grenzen der Kolonie, sondern markieren die Schnittlinie der Kolonie mit Ober- bzw. Unterseite der Probe. Da die Korngrenze eine (001)-Ebene ist, lässt sich aus dem Schnittwinkel zwischen Korngrenze und Kolonie 
in der Projektion auf die Bildebene schließen, dass die Kolonien auf $\{110\}$-Ebenen liegen.

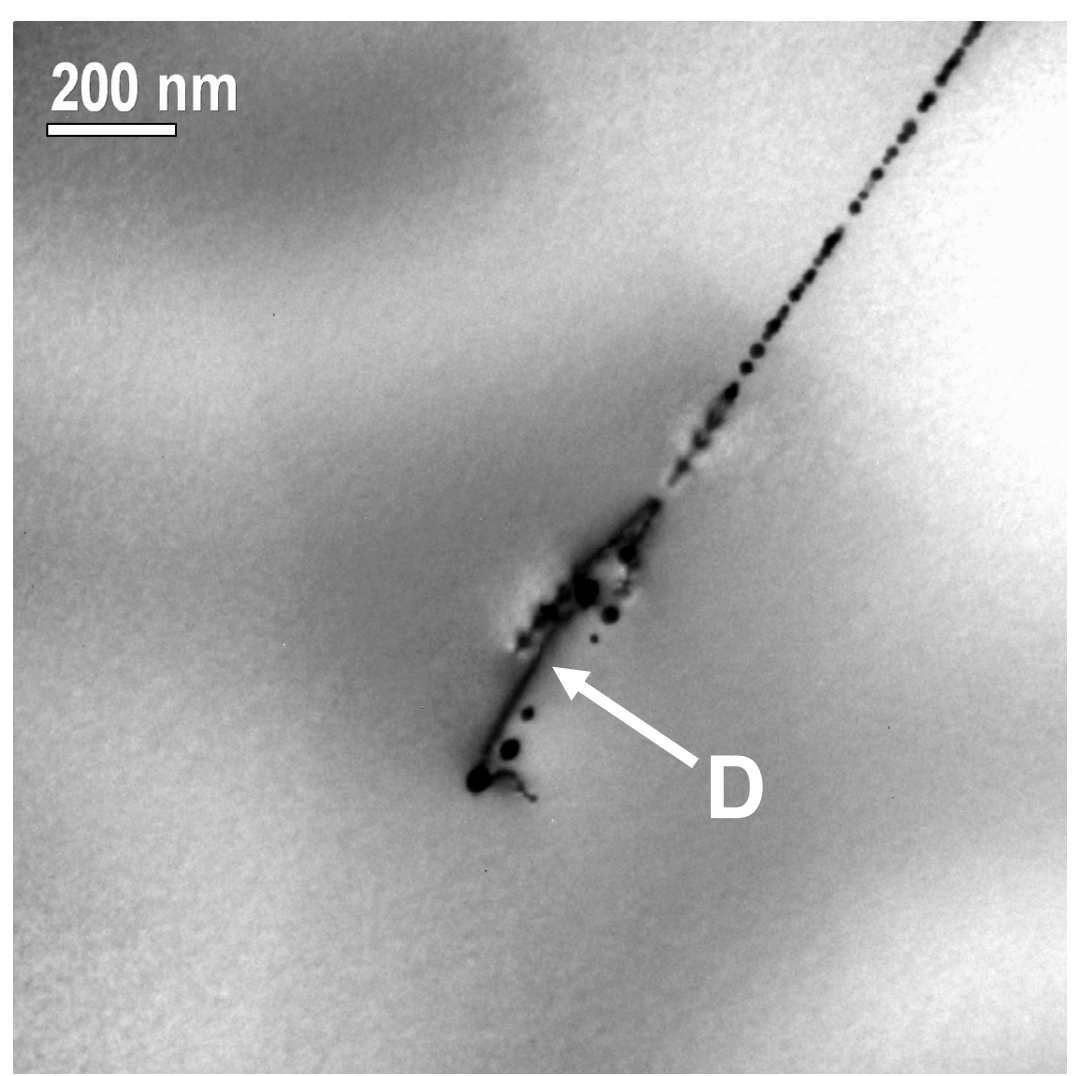

Abbildung 4.5. - TEM-Hellfeldaufnahme des Randbereichs einer Kolonie von Ausscheidungen nach Kodiffusion von $\mathrm{Cu}$ und $\mathrm{Ni}$ bei $1050^{\circ} \mathrm{C}$ und langsamer Abkühlung. Die Ausscheidungen sind auf einer Ebene angeordnet, die hier senkrecht zur Bildebene liegt. Am Rand der Kolonie verläuft eine Versetzung (D).

Die Kolonien werden am Rand von Versetzungen begrenzt, wie Abb.4.5zeigt. Die Versetzungsline verläuft gekrümmt und wird dabei von einer Reihe von Ausscheidungen dekoriert. Kolonien, die aus planaren Anordnungen von Ausscheidungen auf $\{110\}$-Ebenen bestehen und dabei von Versetzungen berandet werden, stellen eine Defektstruktur dar, die typisch ist für Ausscheidungen einer Metallsilizidphase mit starker Volumenfehlpassung. Solche Strukturen wurden beispielsweise beobachtet für Ausscheidungen der $\mathrm{Cu}_{3} \mathrm{Si}$-Phase [42].

Im Rahmen der bisherigen Ergebnisse gleicht die Defektstruktur der Ausscheidungen nach Kodiffusion von $\mathrm{Cu}$ und $\mathrm{Ni}$ der von einfachen $\mathrm{Cu}_{3} \mathrm{Si}$-Ausscheidungen in Si. Elektro- 


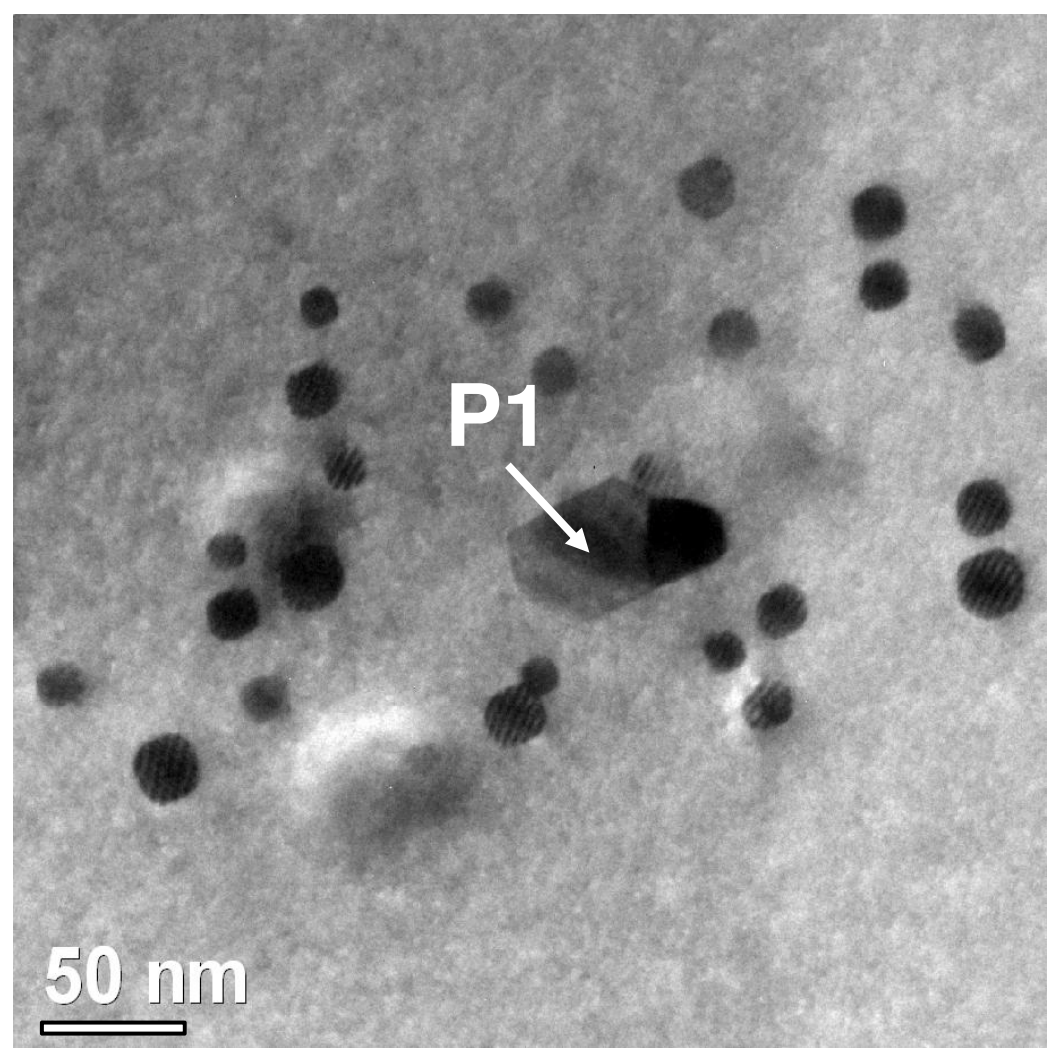

Abbildung 4.6. - TEM-Aufnahme einer Kolonie von Ausscheidungen nach Kodiffusion von Cu und $\mathrm{Ni}$ bei $1050^{\circ} \mathrm{C}$ und langsamer Abkühlung. Mit Ausnahme der mit P1 bezeichneten polyedrischen Ausscheidung sind alle Ausscheidungen nahezu kugelförmig.

nenmikroskopische Aufnahmen in höherer Vergrößerung wie beispielsweise in Abb. 4.6 lassen jedoch erkennen, dass es zwei Typen von Ausscheidungen in den Kolonien gibt.

Zum größten Teil sind die Ausscheidungen nahezu kugelförmig. Ein solches kugelförmiges Teilchen wird in Abb. 4.7 näher untersucht. Einige Teilchen in den Kolonien haben aber eine polyedrische Form mit Oberflächen parallel zu $\{111\}$-Ebenen wie beispielsweise das in Abb. 4.6 mit P1 bezeichnete. Diese Teilchen sind Ausscheidungen einer dritten Phase neben Si und der Phase der kugelförmigen Ausscheidungen. Der Mengenanteil der polyedrischen Ausscheidungen an den Teilchen einer Kolonie insgesamt beträgt etwa 8\%. Sie bilden dabei oft mit anderen Ausscheidungen zusammenhängende Teilchen, wie im Fall des hier mit P1 bezeichneten Teilchens oder des Teilchen P2 in Abb. 4.8, Es gibt auch einzelne von anderen Teilchen getrennte polyedrische Ausscheidungen, wie Abb. 4.9 zeigt. 


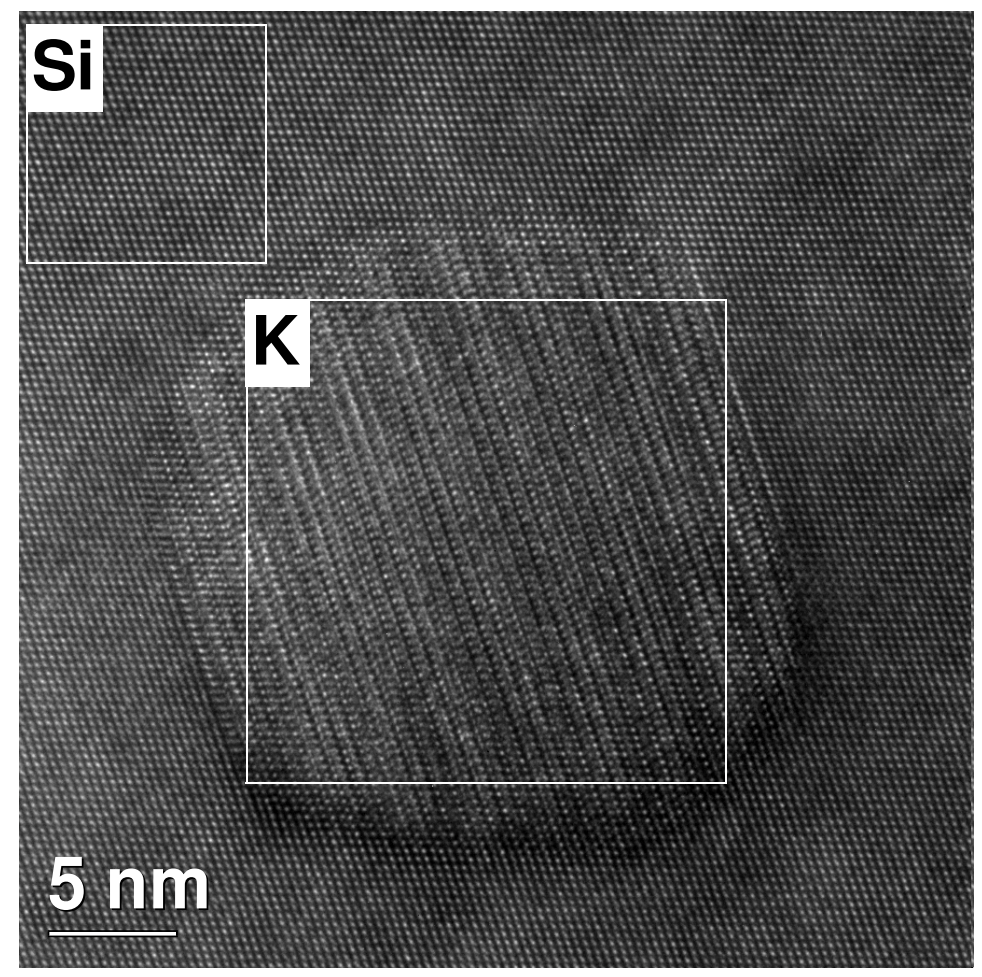

(a)



(b)

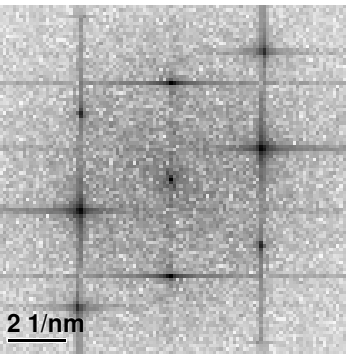

(c)

Abbildung 4.7. - (a) Hochauflösende TEM-Aufnahme einer nahezu kugelförmigen Ausscheidung mit einem Moiré-Muster nach Kodiffusion von $\mathrm{Cu}$ und $\mathrm{Ni}$ bei $1050^{\circ} \mathrm{C}$ und langsamer Abkühlung, (b) Das Diffraktogramm eines Bereiches der Ausscheidung (K) enthält zusätzliche Intensitätsmaxima in der Nähe der Maxima, die im Diffrakrogramm (c) eines Bereiches der Si-Matrix (Si) auftreten. Diese zusätzlichen Maxima deuten auf die Überlagerung von Probenbereichen mit unterschiedlichen Gitterparametern hin. 
Abb.4.7zeigt eine hochauflösende TEM-Aufnahme einer einzelnen nahezu kugelförmigen Ausscheidung (K). In der Abbildung ist im Bereich des Teilchens ein Streifen-Muster zu sehen. Dieses lässt sich als Moiré-Kontrast deuten. Diffraktogramme der mit K und Si bezeichneten Bereiche innerhalb der kugelförmigen Ausscheidung und der Si-Matrix zeigen eine unterschiedliche Intensitätsverteilung. Im Fall der Ausscheidung liegen zusätzliche Intensitätsmaxima in der Nähe der Maxima des Diffraktogramms der Si-Matrix vor. Die zusätzlichen Maxima entsprechen dem Moiré-Streifenmuster in der Abbildung und zeigen, dass hier in der Probe Bereiche mit unterschiedlich großen Gitterparametern der Elementarzelle überlagert sind. Die nahezu kugelförmigen Ausscheidungen bestehen aus einer Phase, die sich strukturell von Si unterscheidet, wie es für Ausscheidungen aus $\mathrm{Cu}_{3} \mathrm{Si}$ der Fall ist.

Die polyedrischen Ausscheidungen unterscheiden sich strukturell von den kugelförmigen. Sie haben eine Kristallstruktur, die der von $\mathrm{NiSi}_{2}$-Ausscheidungen vom Typ A oder Typ B in Si entspricht, wie in Abb. 4.8 und Abb. 4.9 deutlich wird.

Abb. 4.8 zeigt eine hochauflösende TEM-Aufnahme einer kugelförmigen und einer polyedrischen Ausscheidung, die offenbar zusammenhängen. Das kugelförmige Teilchen (K) hat, wie Moiré-Kontrast und Diffraktogramm belegen, die gleichen Eigenschaften wie das einzelne nahezu kugelförmige Teilchen in Abb. 4.7, Im Gegensatz dazu zeigt das Diffraktogramm eines Ausschnitts der Gitterabbildung der polyedrischen Ausscheidung dieselbe Intensitätsverteilung wie das Diffraktogramm der Si-Matrix. Diese polyedrische Ausscheidung besteht aus einer Phase, deren Kristallstruktur kubisch und deren Gitterkonstante nahezu identisch mit der von $\mathrm{Si}$ ist, wie es für Ausscheidungen aus $\mathrm{NiSi}_{2}$ vom Typ A mit kubischer $\mathrm{CaF}_{2}$-Struktur der Fall ist.

Abb. 4.9 zeigt eine hochauflösende TEM-Aufnahme eines einzelnen polyedrischen Teilchens (P3). Zur Entstehung der in der Abbildung sichtbaren Moiré-Streifen tragen im Wesentlichen die im Diffraktogramm markierten Maxima bei $\pm \frac{1}{3}(111)$ bei. Die zusätzlichen Maxima im Diffraktogramm des Teilchens und das Moiré-Muster lassen sich durch das Vorhandensein eines Zwillings auf einer (111)-Ebene erklären. Das Teilchen besteht wie das Teilchen P2 in Abb. 4.8 aus einer Phase mit kubischer Struktur und Si vergleichbarer Gitterkonstante, wobei hier zusätzlich eine Zwillingsorientierung vorliegt, wie es für $\mathrm{NiSi}_{2}$-Ausscheidung vom Typ B zu erwarten ist. 


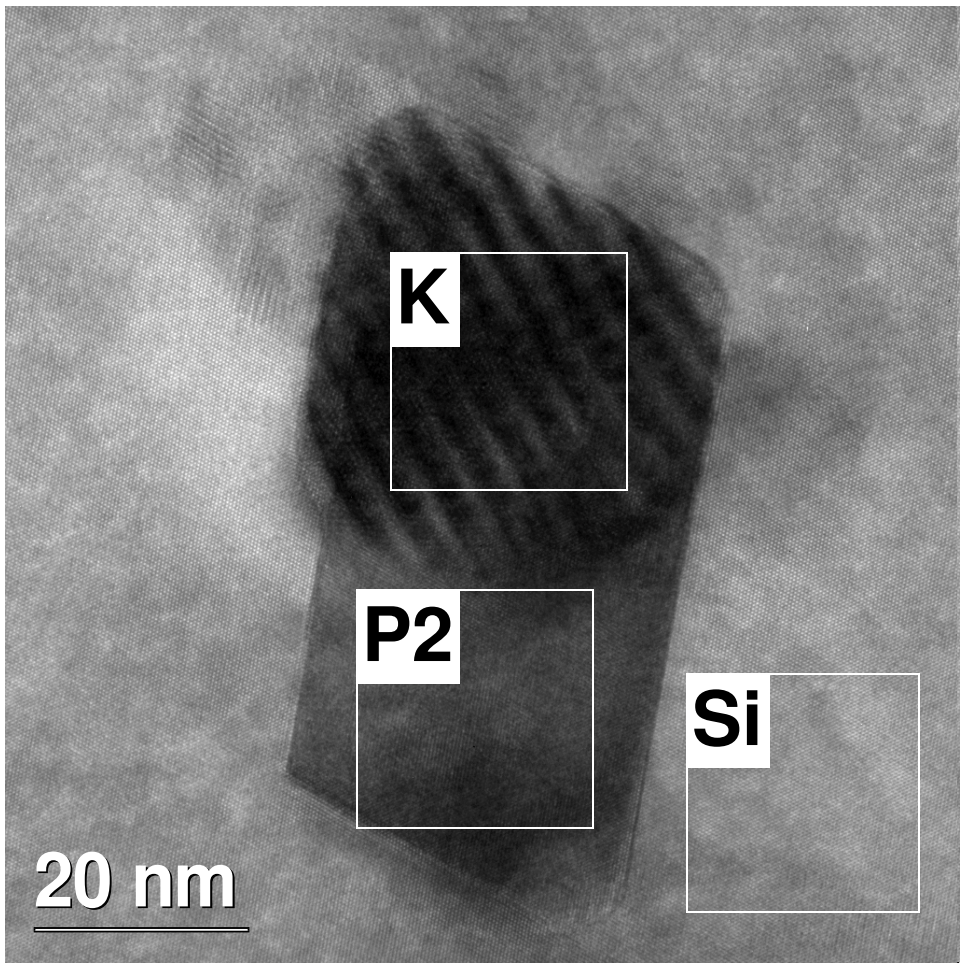

(a)

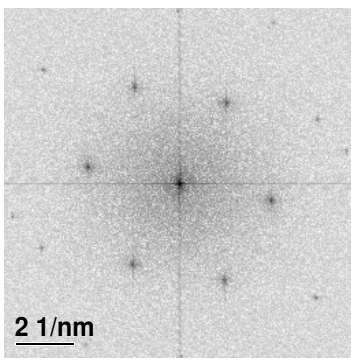

(b)

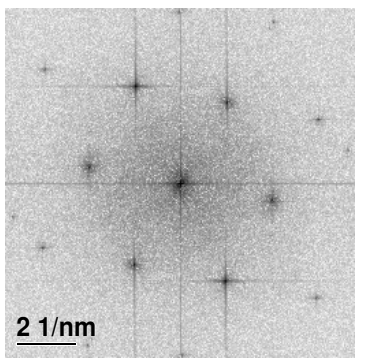

(c)

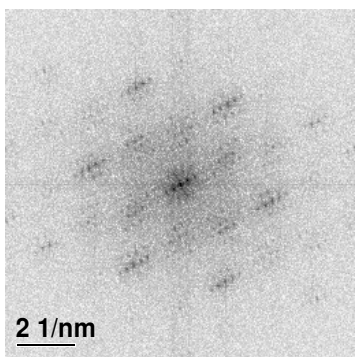

(d)

Abbildung 4.8. - (a) Hochaufösende TEM-Aufnahme zweier zusammenhängender Ausscheidungen nach Kodiffusion von $\mathrm{Cu}$ und $\mathrm{Ni}$ bei $1050^{\circ} \mathrm{C}$ und langsamer Abkühlung. Die Diffraktogramme (b) der polyedrischen Ausscheidung (P2) und (c) von Si zeigen dasselbe Muster, was darauf hinweist, dass die Ausscheidung P2 eine kubische Kristallstruktur mit nahezu gleichgroßer Gitterkonstante wie Si hat. (d) Das Diffraktogramm der zweiten Ausscheidung (K) enthält zusätzliche Intensitätsmaxima, die zeigen, dass die Kristallstruktur dieser Ausscheidung und die von Si unterschiedlich große Gitterparameter besitzen. 


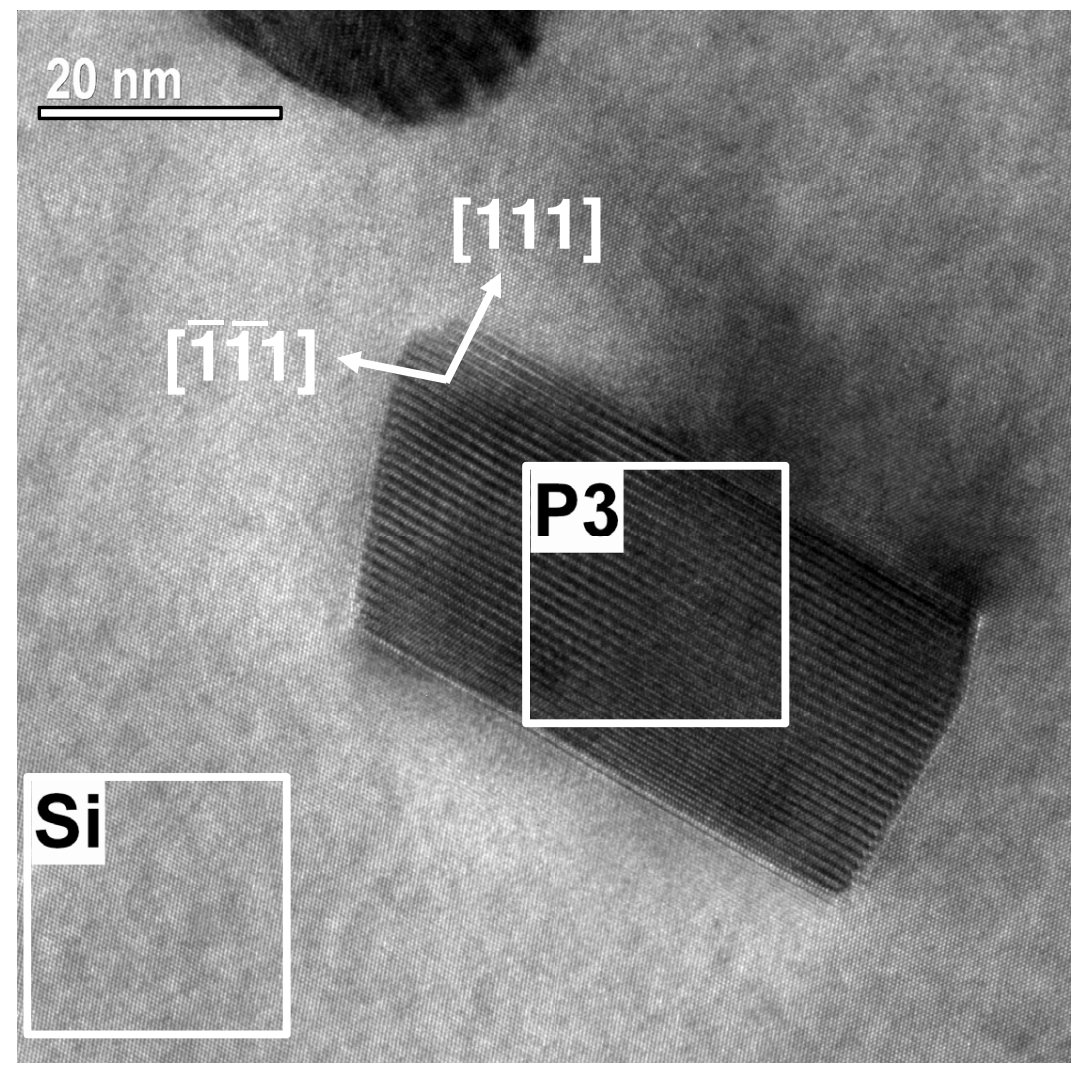

(a)

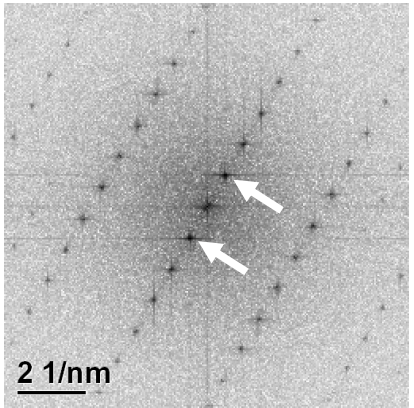

(b)

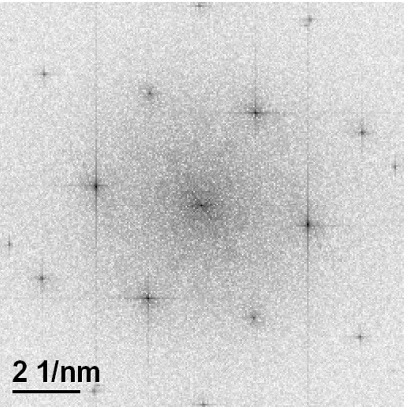

(c)

Abbildung 4.9. - (a) Hochaufösende TEM-Aufnahme einer einzelnen polyedrischen Ausscheidung (P3) nach Kodiffusion von $\mathrm{Cu}$ und $\mathrm{Ni}$ bei $1050^{\circ} \mathrm{C}$ und langsamer Abkühlung mit einem Moiré-Kontrast, der im Wesentlichen durch die im Diffraktogramm (b) der Ausscheidung markierten Intensitätsmaxima zustande kommt. Die im Verglich zum Diffraktogramms der Matrix (c) zusätzlichen Maxima zeigen, dass die Ausscheidung bezüglich der Matrix eine Zwillingsorientierung besitzt. 


\subsubsection{Zusammensetzung}

Nach Kodiffusion von $\mathrm{Cu}$ und $\mathrm{Ni}$ in $\mathrm{Si}$ unter kupferreichen Bedingungen und langsamer Abkühlung lässt sich die Bildung ausgedehnter Kolonien kugelförmiger und polyedrischer Ausscheidungen beobachten. Abb.4.10 stellt das Ergebnis einer EDX-Untersuchung dar, die an der Probenstelle durchgeführt wurde, an der sich die in Abb. 4.6 betrachteten kugelförmigen Ausscheidungen und die polyedrische Ausscheidung P1 befinden. EDXKarten der charakteristischen $\mathrm{K}_{\alpha}$-Röntgenstrahlung von $\mathrm{Cu}$ und $\mathrm{Ni}$ zeigen direkt, dass in den kugelförmigen Teilchen $\mathrm{Cu}$ und in dem polyedrischen Teilchen Ni enthalten ist. Die

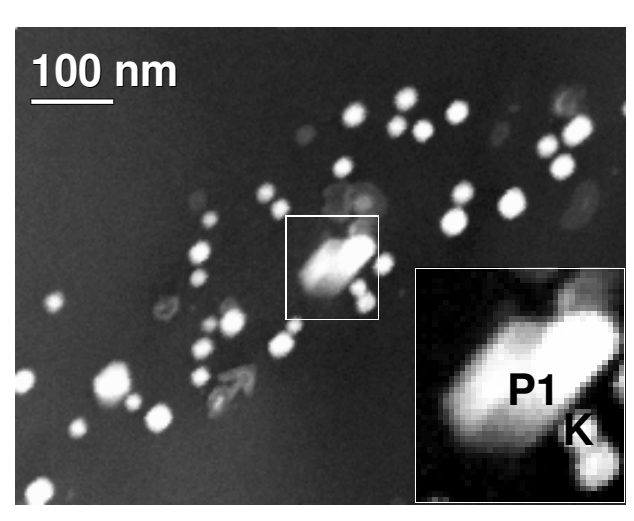

(a) STEM

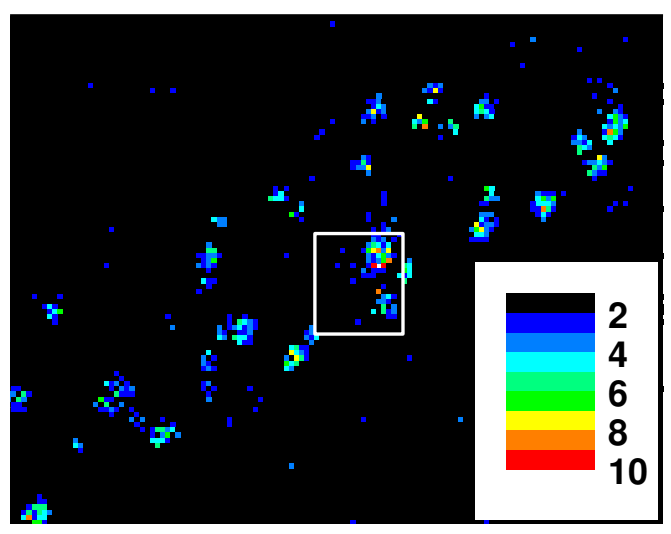

(c) EDX: $\mathrm{Cu}_{K \alpha}$-Signal

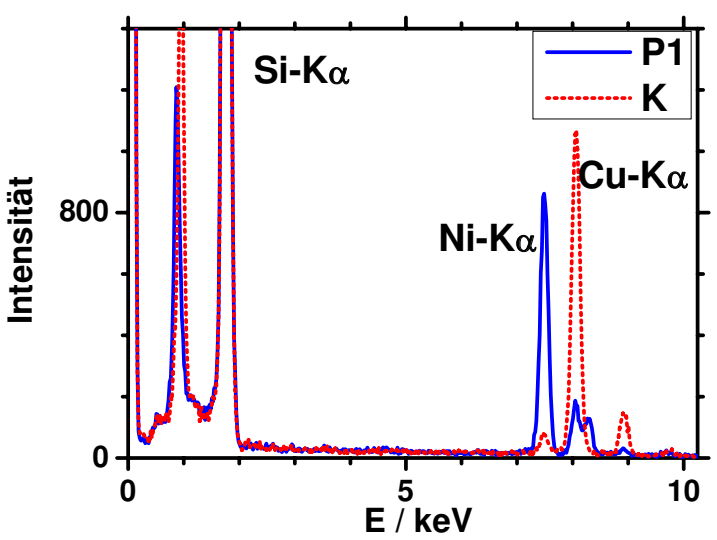

(b) EDX: Spektren

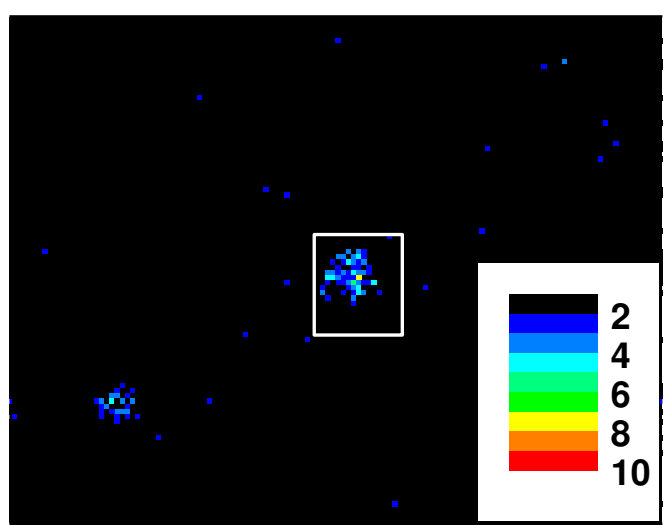

(d) EDX: $\mathrm{Ni}_{K \alpha}-$ Signal

Abbildung 4.10. - Ausscheidungen nach Kodiffusion von $\mathrm{Cu}$ und $\mathrm{Ni}$ bei $1050^{\circ} \mathrm{C}$ und langsamer $\mathrm{Ab}$ kühlung. (a) Rastertransmissionselektronenmikroskopische Abbildung (STEM) der Ausscheidungen, (b) Punktmessungen von EDX-Spektren an den mit P1 und $K$ bezeichneten Ausscheidungen, (c) und (d) laterale Intensitätverteilung der $\mathrm{Cu}_{K \alpha^{-}}$und $\mathrm{Ni}_{K \alpha}$-Linien detektiert während der Abtastung der Probenstelle mit dem Elektronenstrahl. 
Probe war auf einem nicht aus $\mathrm{Cu}$ bestehendem TEM-Netzchen befestigt, um zu verhindern, dass Elektronen, die durch Rück- oder Mehrfachstreuung aus der ursprünglichen Strahlrichtung abgelenkt werden und mit der Materie des TEM-Netzchen wechselwirken könnten, ebenfalls $\mathrm{Cu}_{K \alpha}$-Röntgenstrahlung erzeugen.

Es handelt sich bei den Teilchen um Ausscheidungen einer $\mathrm{Cu}$ - und einer Ni-SilizidPhase. Punktmessungen kompletter EDX-Spektren zeigen außerdem, dass die mit P1 bezeichnete Ausscheidung einer Ni-Silizid-Phase zusätzlich $\mathrm{Cu}$ enthält und die mit K bezeichnete Ausscheidung einer Cu-Silizid-Phase zusätzliches Ni.

Die Ergebnisse dieser EDX-Messungen lassen sich zusammen mit den Ergebnissen der strukturellen Untersuchungen im vorangegangenen Abschnitt 4.2.1.2 so interpretieren, dass die Ausscheidungen aus den bekannten binären Phasen $\mathrm{Cu}_{3} \mathrm{Si}$ und $\mathrm{NiSi}_{2}$ bestehen, wobei diese Phasen nicht in reiner Form vorliegen, sondern außerdem Atome der jeweils anderen metallischen Verunreinigung in gelöster Form enthalten. Diese Phasen werden im Weiteren mit $\mathrm{Cu}_{3} \mathrm{Si}: \mathrm{Ni}$ bzw. $\mathrm{NiSi}_{2}: \mathrm{Cu}$ bezeichnet.

Als Messgröße für die Zusammensetzung dient in dieser Arbeit vorwiegend der partielle Molenbruch $X_{C u}^{(p)}$ von $\mathrm{Cu}$. Hierbei ist

$$
X_{C u}^{(p)}=\frac{[C u]}{[C u]+[N i]},
$$

wobei $[\mathrm{Cu}]$ und $[\mathrm{Ni}]$ die Konzentrationen von $\mathrm{Cu}$ und $\mathrm{Ni}$ sind.

Der Anteil eines einzelnen Elements beispielsweise von Kupfer am EDX-Spektrum lässt sich nach der Methode von Cliff und Lorimer quantifizieren [78]. Dieser Anteil entspricht bei einer in Richtung des Elektronenstrahl homogen aufgebauten Probe dem Molenbruch $X_{C u}$ von Kupfer. Bei Ausscheidungen jedoch, deren Größe geringer ist als die Probendicke, trägt auch die Si-Matrix einen Beitrag zum Si-Signal im EDX-Spektrum bei, so dass die genaue Zusammensetzung kleiner Teilchen unter Berücksichtigung des Si-Anteils nicht bestimmt werden kann. In diesem Fall dienen die nach Cliff und Lorimer [78] erhaltenen Werte für $X_{C u}$ und $X_{N i}$ als Ausgangswerte für die Berechnung von $X_{C u}^{(p)}$. Im Fall der beiden in Abb. 4.10 näher bezeichneten Ausscheidungen beispielsweise beträgt der partielle Molenbruch von $\mathrm{Cu} 15(2) \%$ in der $\mathrm{NiSi}_{2}: \mathrm{Cu}$-Ausscheidung P1 und 95(2)\% in der $\mathrm{Cu}_{3} \mathrm{Si}$ :Ni-Ausscheidung $\mathrm{K}$.

Nun soll die Zusammensetzung zusammenhängender Ausscheidungen näher betrachtet werden. Abb. 4.11 zeigt das Ergebnis einer EDX-Messung entlang einer Strecke durch zwei solcher Ausscheidungen, die in Abb4.8 mit hochauflösender TEM untersucht worden sind. Diese Messung zeigt, dass auch zusammenhängende Ausscheidungen der beiden unterschiedlichen Phasen jeweils beide metallische Verunreinigungen enthalten.

Abb. 4.12 stellt den Verlauf des partiellen Molenbruchs $X_{C u}^{(p)}$ von $\mathrm{Cu}$ dar, der sich 


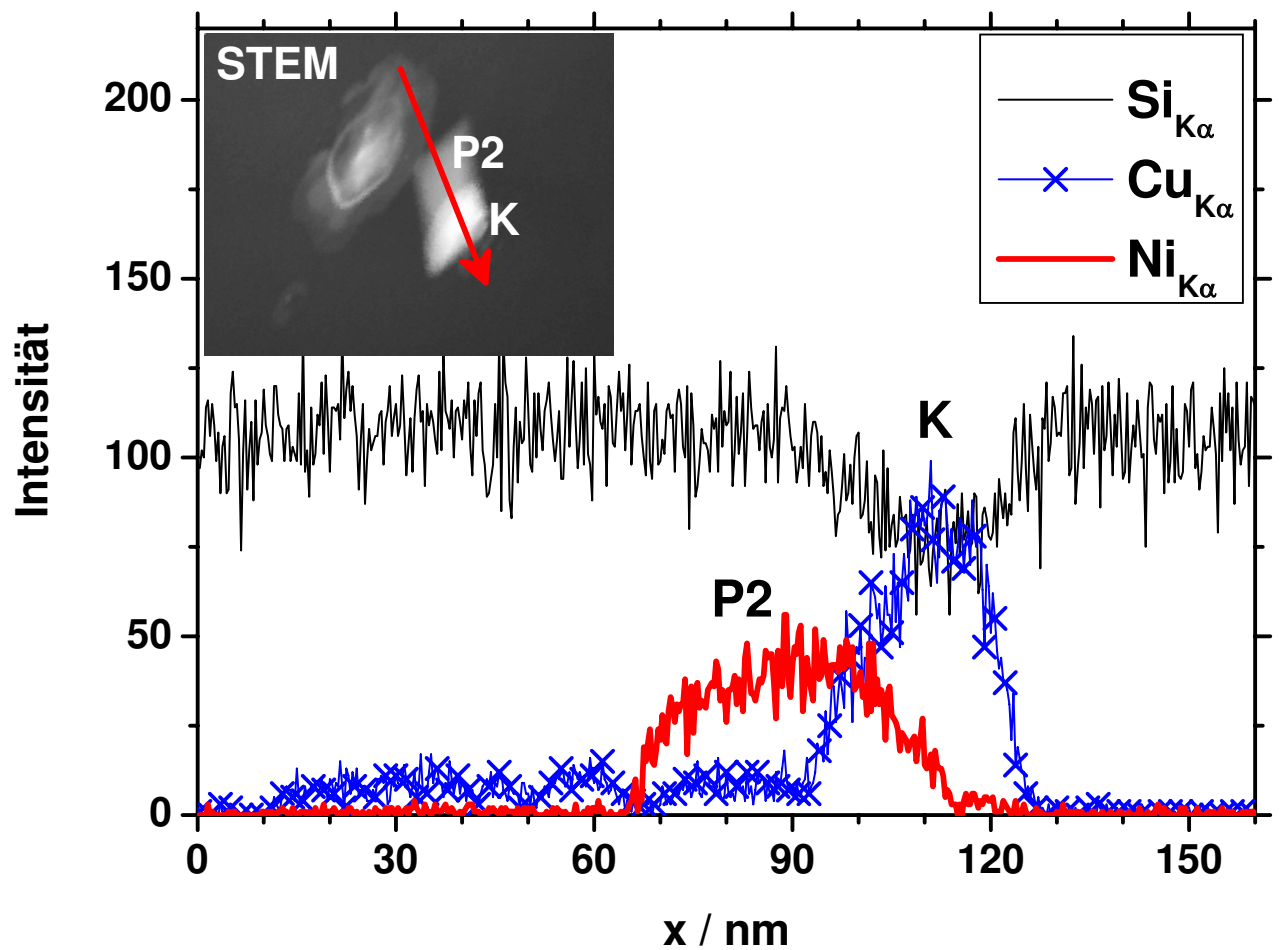

Abbildung 4.11. - EDX-Messung an zwei zusammenhängenden Ausscheidungen nach Kodiffusion von $\mathrm{Cu}$ und $\mathrm{Ni}$ bei $1050^{\circ} \mathrm{C}$ und langsamer Abkühlung. Dargestellt sind die unkorrigierten Intensitäten der $\mathrm{Si}_{K \alpha^{-}}, \mathrm{Ni}_{K \alpha^{-}}$und $\mathrm{Cu}_{K \alpha^{-}}$Linien, die gemessen wurden, während der Elektronenstrahl durch die Ausscheidungen hindurch bewegt wurde (STEM). Die Intensität der Si Ka-Linie nimmt im Bereich der $\mathrm{Cu}_{3} \mathrm{Si}: \mathrm{Ni}$ Ausscheidung (K) gegenüber der Si-Matrix signifikant ab, wohingegen sie im Bereich der $\mathrm{NiSi}_{2}: \mathrm{Cu}$-Ausscheidung (P2) unverändert ist.

aus den in Abb. 4.11 gezeigten EDX-Signalen bei einer Messung auf einer Linie entlang zweier zusammenhängender Ausscheidungen ergibt:

- Im Bereich des $\mathrm{NiSi}_{2}$ :Cu-Teilchens schwankt der Wert von $X_{C u}^{(p)}$ um $15 \%$.

- Im Bereich des Überlapps der beiden Ausscheidungen zwischen $x=95 \mathrm{~nm}$ und $x=110 \mathrm{~nm}$ steigt der Wert von $X_{C u}^{(p)}$ von 15 auf $95 \%$.

- Im Bereich der $\mathrm{Cu}_{3} \mathrm{Si}$-Ausscheidung schwankt der Wert $X_{C u}^{(p)}$ um $95 \%$.

Es zeigt sich, dass bei EDX-Punktmessungen an Teilchen, die aus zwei zusammenhängenden Ausscheidungen verschiedener Phasen bestehen, von denen eine kupfer- und die 


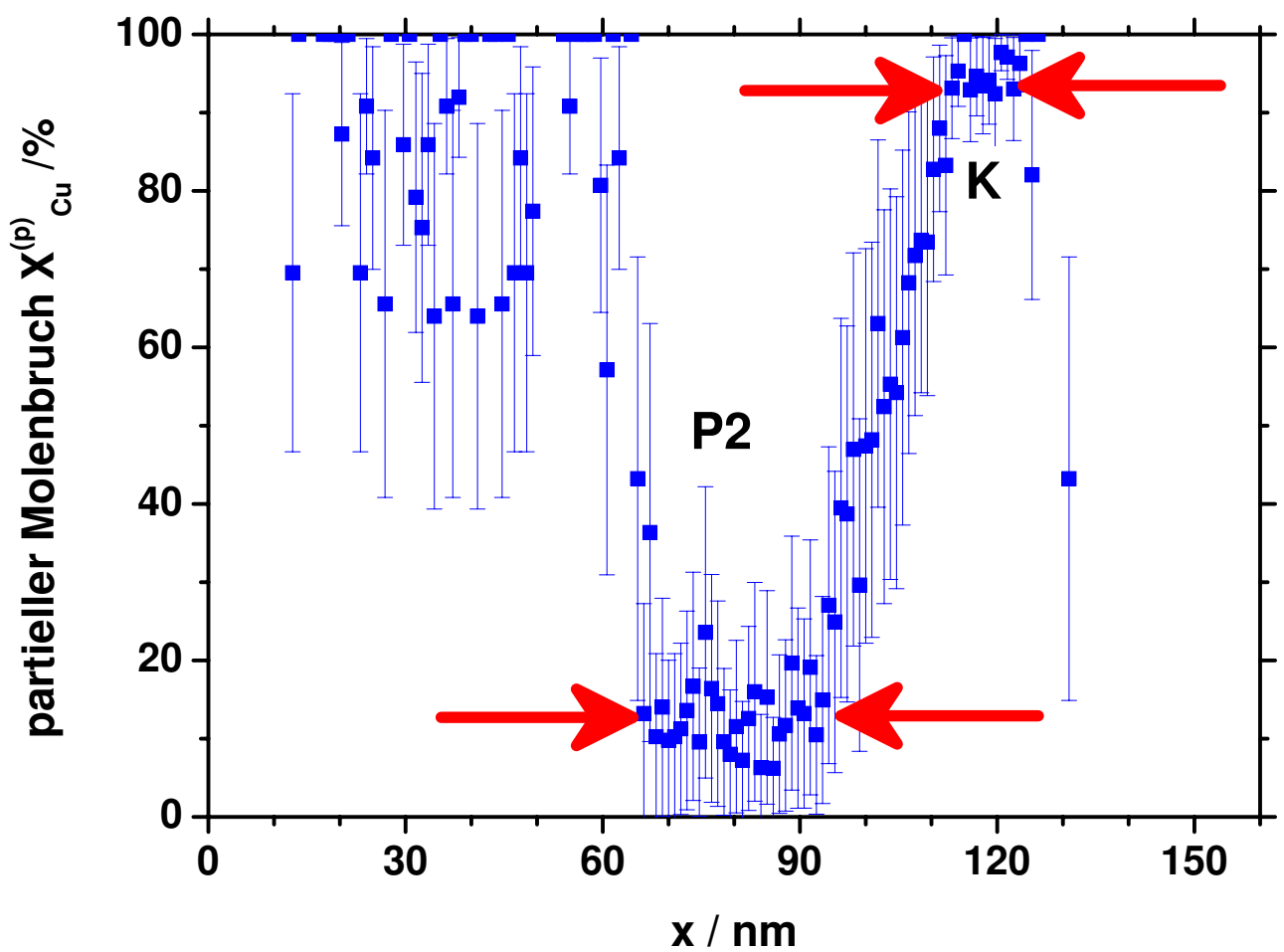

Abbildung 4.12. - Partieller Molenbruch $X_{C u}^{(p)}$ von $C u$ gemessen entlang eines Schnitts durch zwei zusammenhängende Ausscheidungen (P2 und $\mathrm{K}$ ) der $\mathrm{NiSi}_{2}: \mathrm{Cu}$-Phase (P2) und der $\mathrm{Cu}_{3} \mathrm{Si}$ :Ni-Phase $(\mathrm{K})$ nach Kodiffusion von $\mathrm{Cu}$ und $\mathrm{Ni}$ bei $1050^{\circ} \mathrm{C}$ und langsamer Abkühlung. Die Werte von $X_{C u}^{(p)}$ wurde aus den in $A b b$. 4.11 dargestellten Intensitäten der $\mathrm{Ni}_{K \alpha^{-}}$und $\mathrm{Cu}_{K \alpha}$-Linien berechnet.

andere nickelreich ist, der Bereich zu beachten ist, in dem die beiden Ausscheidungen überlappen. Bei einer Messung an einer willkürlich festgelegten Stelle kann der gemessene Wert des partiellen Molenbruchs von $\mathrm{Cu}$ jeden Wert zwischen denen der $\mathrm{NiSi}_{2}: \mathrm{Cu}-$ und der $\mathrm{Cu}_{3} \mathrm{Si}$ :-Ausscheidung annehmen.

Die EDX-Messung in Abb. 4.11 lässt auch Rückschlüsse auf die Verteilung von Si in den beiden zusammenhängenden Ausscheidungen zu. Im Bereich des $\mathrm{Cu}_{3} \mathrm{Si}$ :Ni-Teilchens verringert sich die Intensität der $\mathrm{Si}_{K \alpha}$-Linie, während sie im Bereich des $\mathrm{NiSi}_{2}: \mathrm{Cu}-$ Teilchen konstant bleibt. Dieses Ergebnis zeigt, dass die Dichte der Si-Atome in der Phase der $\mathrm{Cu}_{3} \mathrm{Si}$ :Ni-Ausscheidung geringer ist als in $\mathrm{Si}$, was sich als Hinweis auf die Erzeugung interstitieller Si-Atome beim Wachstum der Ausscheidung interpretieren lässt. Dieses Verhalten ist aufgrund der hohen Volumenfehlpassung von $\mathrm{Cu}_{3} \mathrm{Si}$ zu erwarten. 


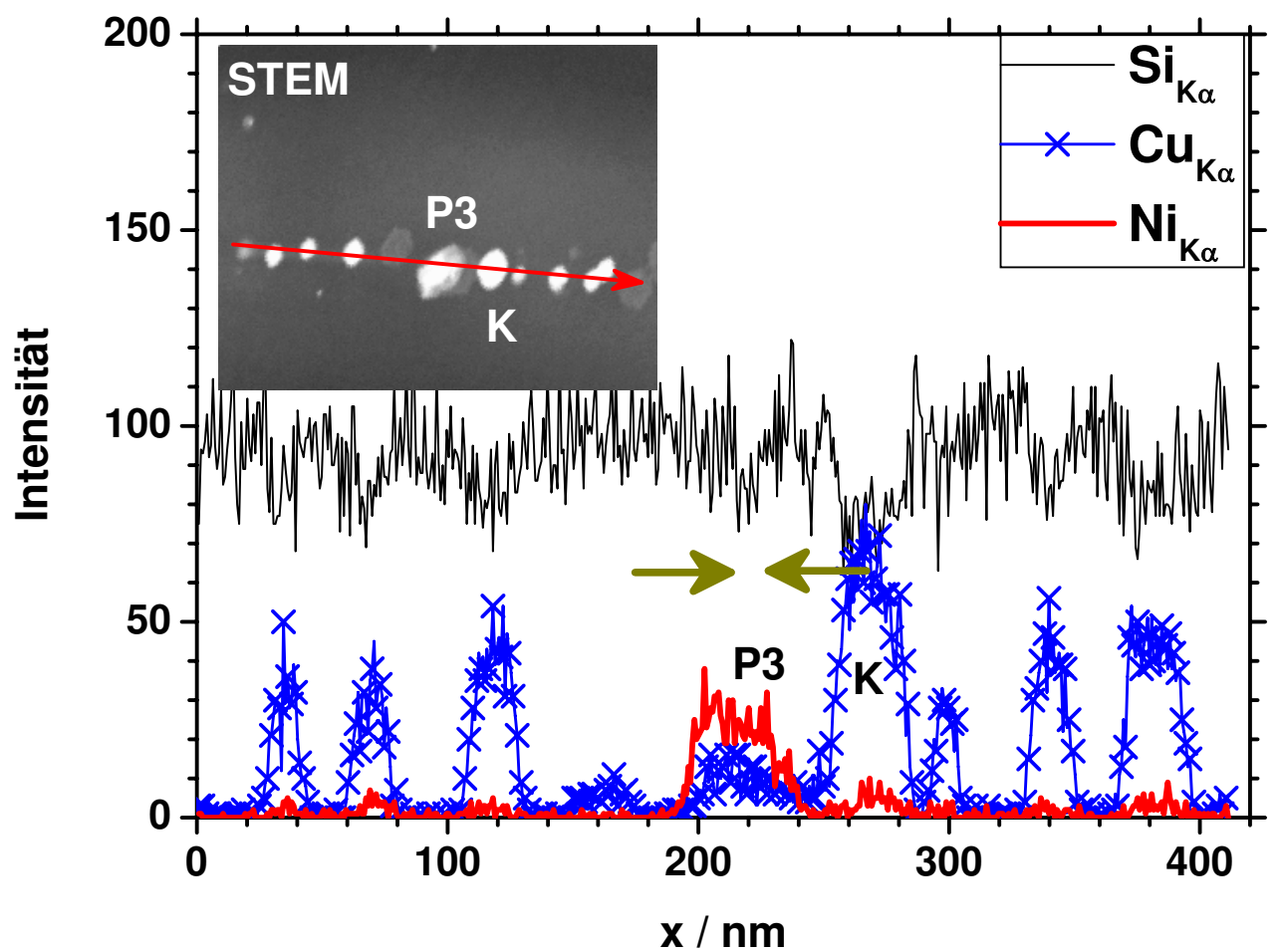

Abbildung 4.13. - EDX-Messung an einer Kolonie von Ausscheidungen nach Kodiffusion von $\mathrm{Cu}$ und Ni bei $1050^{\circ} \mathrm{C}$ und langsamer Abkühlung. Dargestellt sind die unkorrigierten Intensitäten der $\mathrm{Si}_{K \alpha^{-}}, \mathrm{Ni}_{K \alpha^{-}}$und $\mathrm{Cu}_{K \alpha^{-}}$Linien, die gemessen wurden, während der Elektronenstrahl wie im STEM-Bild angezeigt entlang der Kolonie bewegt wurde. Jede Ausscheidung enthält sowohl $\mathrm{Cu}$ als auch Ni. Ausscheidungen der $\mathrm{Cu}_{3} \mathrm{Si}$ :Ni-Phase (z.B. K) sind verbunden mit einer Verringerung der Intensität der $\mathrm{Si}_{\mathrm{K \alpha}}$-Linie. Die $\mathrm{NiSi}_{2}$ :Cu-Ausscheidung (P3) zeigt innerhalb des markierten Bereiches ein ähnliches Verhalten.

Im Bereich der $\mathrm{NiSi}_{2}$ :Cu-Ausscheidung hingegen ist die Dichte der Si-Atome im Rahmen der Messgenauigkeit gleich der Dichte der Gitterplätze in Si. Diese Beobachtung ist für reine $\mathrm{NiSi}_{2}$-Ausscheidungen zu erwarten, da $\mathrm{NiSi}_{2}$ verglichen mit $\mathrm{Cu}_{3} \mathrm{Si}$ eine sehr geringe Fehlpassung zu Si aufweist.

Abb. 4.13 stellt das Ergebnis einer weiteren EDX-Messung entlang einer Strecke in einer Kolonie dar, die in Abb. 4.9 mit hochauflösender TEM untersuchte Ausscheidung P3 enthält. Im Bereich des $\mathrm{Cu}_{3} \mathrm{Si}$ :Ni-Teilchens $\mathrm{K}$ verringert sich die Intensität der $\mathrm{Si}_{K \alpha^{-}}$ Linie, was sich analog zu der $\mathrm{Cu}_{3} \mathrm{Si}$ :Ni-Ausscheidung in Abb. 4.11 als Verdrängung von Si aus der wachsenden Ausscheidung aufgrund hoher Volumenfehlpassung deuten lässt. 


\begin{tabular}{|c|c|c|c|c|c|}
\hline \multirow{2}{*}{ Ausscheidung } & $X_{C u}^{(p)}(\%)$ & \multicolumn{2}{|c|}{ Referenz } & \multicolumn{2}{c|}{$X_{C u}$ (At.\%) } \\
& & TEM & EDX & Cu auf Ni-Platz & Cu auf Si-Platz \\
\hline P1 & $14.9 \pm 2.5$ & Abb. 4.6 & Abb. 4.10 & $5.0 \pm 0.9$ & $5.8 \pm 1.2$ \\
P2 & $14.6 \pm 3.1$ & Abb. 4.8 & Abb. 4.11 & $4.9 \pm 1.1$ & $5.7 \pm 1.5$ \\
P3 & $22.5 \pm 3.2$ & Abb. 4.9 & Abb. 4.13 & $7.5 \pm 1.1$ & $9.7 \pm 1.8$ \\
\hline
\end{tabular}

Tabelle 4.3. - Zusammensetzung von $\mathrm{NiSi}_{2}$ :Cu-Ausscheidungen nach Kodiffusion von $\mathrm{Cu}$ und $\mathrm{Ni}$ unter kupferreichen Bedingungen. Angegeben ist jeweils der partielle Molenbruch $X_{C u}^{(p)}$ von $\mathrm{Cu}$, vgl. Gl. (4.2), der sich aus der quantitativen Analyse eines EDX-Spektrums nach einer Punktmessung ergibt, sowie der theoretische Wert des Molenbruchs $X_{C u}$ unter der Annahme, dass $\mathrm{Cu}$ in $\mathrm{NiSi}_{2}: \mathrm{Cu}$-Ausscheidungen entweder auf $\mathrm{Ni}$ - oder auf Si-Plätzen der $\mathrm{NiSi}_{2}$-Struktur eingebaut ist.

In einem Teil der $\mathrm{NiSi}_{2}$ :Cu-Ausscheidung P3 kann eine Verringerung der Intensität $I_{S i}$ der $\mathrm{Si}_{K \alpha}$-Linie beobachtet werden. Zur Einschätzung der Signifikanz dieser Verringerung kann die Abweichung $\Delta I_{S i}$ der Intensität $I_{S i}$ vom mittleren Wert in der Si-Matrix betrachtet werden. Der Mittelwert von $I_{S i}$ in der Matrix beträgt bei dieser Messung $97 \pm(1)$ Zählereignisse mit einer Standardabweichung $\sigma_{M}$ von 10 Zählereignissen. Die Verringerung $\Delta I_{S i}$ innerhalb des markierten Bereichs der Ausscheidung P3 gegenüber der Matrix liegt in der Größenordnung einer Standardabweichung $\sigma_{M}$, während $\Delta I_{S i} \mathrm{im}$ Fall der kupferreichen Ausscheidung K ungefähr zwei $\sigma_{M}$ beträgt.

Tabelle 4.3 fasst die Werte für den partiellen Molenbruch $X_{C u}^{(p)}$ von $\mathrm{Cu}$ in den drei betrachteten $\mathrm{NiSi}_{2}$ :Cu-Ausscheidungen zusammen. Außerdem ist der absolute Molenbruch $X_{C u}$ von $\mathrm{Cu}$ angegeben, der sich bei Annahme einer Zusammensetzung ausgehend von $\mathrm{NiSi}_{2}$ ergibt, wenn die Cu-Atome substitutionell auf Ni-Plätzen oder auf Cu-Plätzen eingebaut sind. Die Werte des partiellen Molenbruchs sind unterschiedlich. Zwei Teilchen (P1 und P2), die Typ-A-Orientierung zeigen und an $\mathrm{Cu}_{3} \mathrm{Si}$ Ni-Teilchen grenzen, haben jeweils einen niedrigeren $\mathrm{Cu}$-Anteil als ein Teilchen (P3), das von anderen Ausscheidungen getrennt ist und Typ-B-Orientierung besitzt.

Bei den bisher untersuchten Ausscheidungen handelt es sich einzelne oder zusammenhängende Teilchen aus unterschiedlichen Kolonien. Zum Ende dieses Abschnitts soll die Zusammensetzung, die laterale Verteilung und das Anzahlverhältnis von $\mathrm{NiSi}_{2}: \mathrm{Cu}-$ und $\mathrm{Cu}_{3} \mathrm{Si}$ :Ni-Ausscheidungen innerhalb einer einzelnen Kolonie genauer untersucht werden. Dazu wurden Punktmessungen an 154 Ausscheidungen in einem 8.4x0.1 $\mu \mathrm{m}^{2}$ großen Gebiet einer Kolonie vorgenommen und jeweils der partielle Molenbruch von Cu bestimmt. Die bisher betrachteten $\mathrm{NiSi}_{2}$ :Cu-Ausscheidungen P1, P2 und P3 liegen nicht innerhalb dieser Kolonie. Abb. 4.14 zeigt, dass die Häufigkeitsverteilung des Wertes des partiellen Molenbruchs ein eindeutiges Maximum auf der kupferreichen Seite hat. An die Verteilung kann numerisch eine Gaussverteilung mit dem Maximum bei $96.8 \pm 0.2 \%$ 


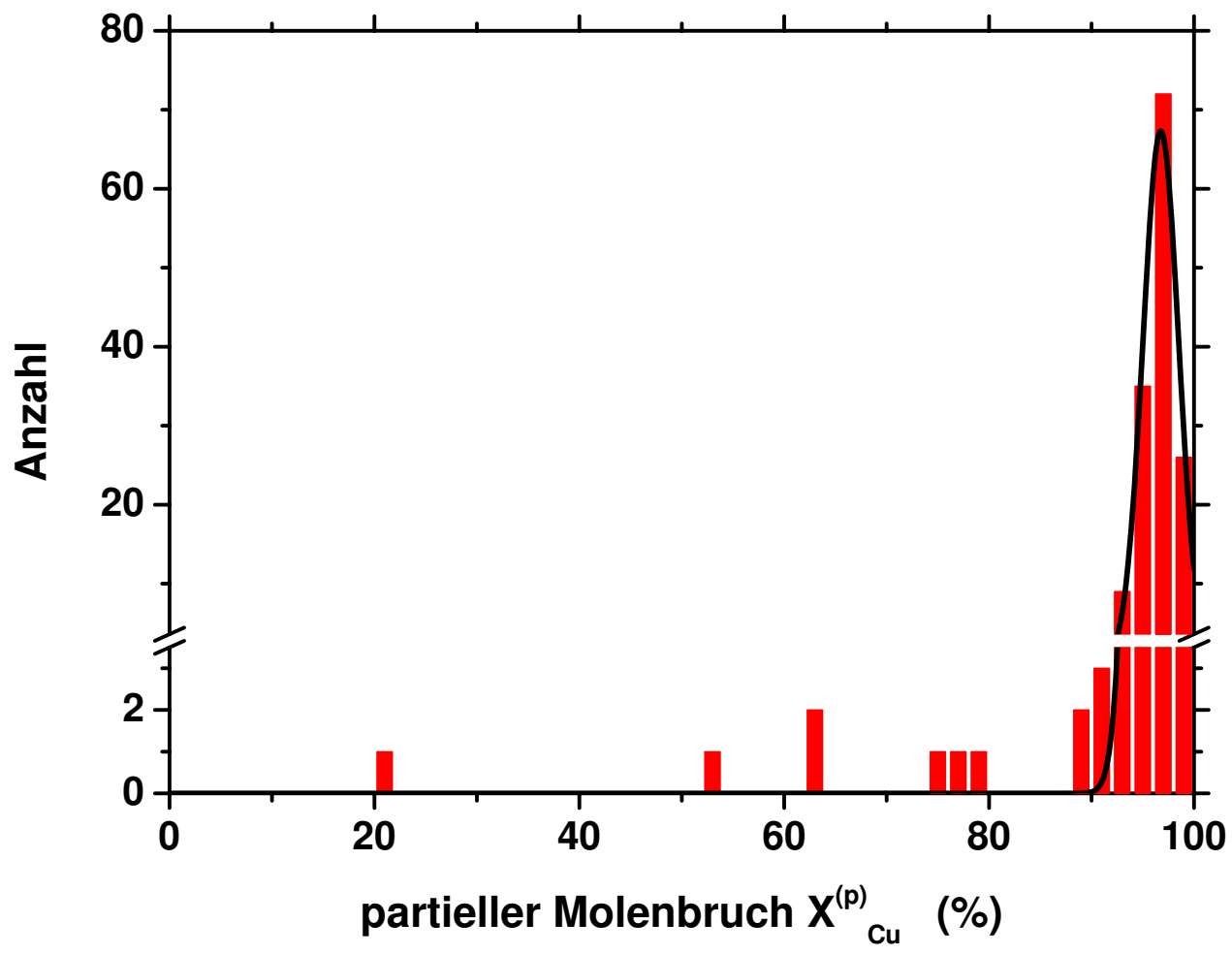

Abbildung 4.14. - Häufigkeitsverteilung des partiellen Molenbruchs $X_{C u}^{(p)}$ von $\mathrm{Cu}$ in Ausscheidungen nach Kodiffusion von $\mathrm{Cu}$ und $\mathrm{Ni}$ bei $1050^{\circ} \mathrm{C}$ und langsamer Abkühlung. Für $\mathrm{Cu}_{3} \mathrm{Si}$ :Ni-Ausscheidungen mit $X_{C u}^{(p)}>91.2 \%$ kann die Häufigkeitsverteilung durch eine Gaußsche Verteilungsfunktion mit dem Maximum bei $96.8 \pm 0.2 \%$ und einer Standardabweichung von $1.7 \pm 0.2 \%$ angepasst werden. Der partielle Molenbruch wurde für 154 Ausscheidungen aus einem $8.4 \mathrm{x} 0.1 \mu \mathrm{m}^{2}$ großen Bereich einer Kolonie durch EDX-Punktmessungen bestimmt.

und einer Standardabweichung von $1.7 \pm 0.2 \%$ angepasst werden. Dazu ist es erforderlich die Werte aus Messungen an $\mathrm{Cu}_{3} \mathrm{Si}$ :Ni-Teilchen gegenüber Werten abzugrenzen, die von $\mathrm{NiSi}_{2}: \mathrm{Cu}$-Teilchen oder zusammenhängenden Ausscheidungen verschiedener Phasen herrühren. Teilchen mit $X_{C u}^{(P)}<90 \%$ lassen sich direkt ausschließen. Als weiteres Kriterium kann

$$
X_{C u}^{(P)}>91.2 \%
$$

benutzt werden. Werte kleiner als $91.2 \%$ liegen außerhalb des $3 \sigma$-Intervalls um den Mittelwert $m$ der verbeibenden Werte für $X_{C u}^{(P)}>90 \%$. Dabei beträgt der Mittelwert $m=96.5 \%$ und die Standardabweichung $\sigma=1.7 \%$. 


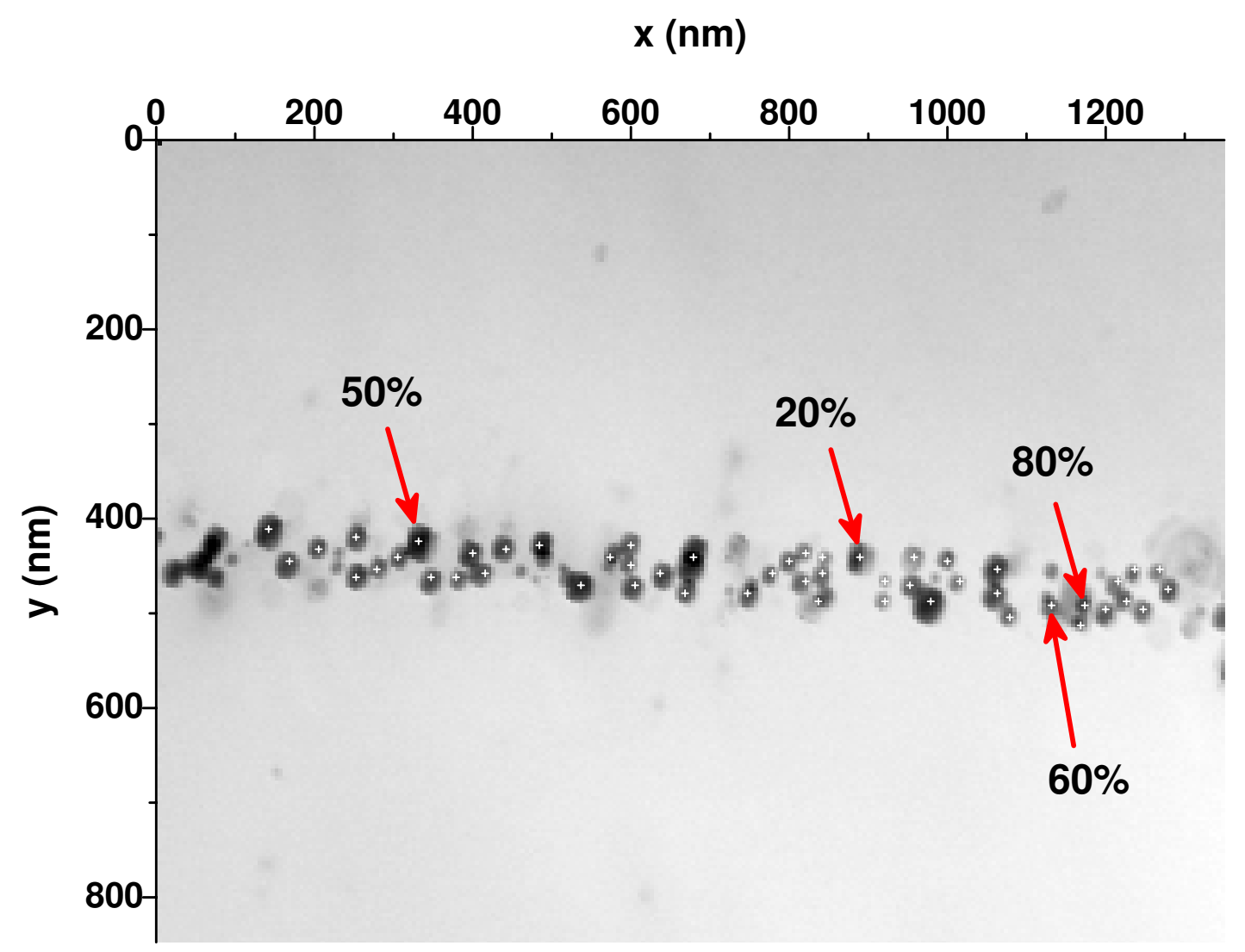

Abbildung 4.15. - STEM-Aufnahme eines Teils der Kolonie, für deren Ausscheidungen Abb. 4.14 die Häufigkeitsverteilung des partiellen Molenbruchs $X_{C u}^{(p)}$ von $\mathrm{Cu}$ zeigt. $\mathrm{NiSi}_{2}: \mathrm{Cu}$-Ausscheidungen liegen an den mit Pfeilen markierten Stellen. Die Zahlen geben die mit EDX gemessenen Werte von $X_{C u}^{(p)}$ an. Werte im Bereich $50 \%<X_{\mathrm{Cu}}^{(p)}<90 \%$ deuten auf zusammenhängende $\mathrm{NiSi}_{2}: \mathrm{Cu}$ - und $\mathrm{Cu}_{3} \mathrm{Si}: \mathrm{Ni}$ Teilchen hin, deren EDX-Signale sich überlagern.

Definiert man für den partiellen Molenbruch $X_{C u}^{(p)}$ von $\mathrm{Cu}$ in $\mathrm{Cu}_{3} \mathrm{Si}$ :Ni-Ausscheidungen als untere Grenze eine Wert von 91.2\%, bleiben insgesamt 12 Ausscheidungen mit niedrigeren Werten übrig. Dies entspricht einem Mengenanteil der $\mathrm{NiSi}_{2}: \mathrm{Cu}$-Ausscheidungen an den Teilchen insgesamt von etwa 8\%. Diese Werte des partiellen Molenbruchs von $\mathrm{Cu}$ ergeben kein eindeutiges Maximum auf der nickelreichen Seite, sondern sind relativ breit verteilt. Sie lassen sich als Messwerte an Stellen interpretieren, an denen EDX-Signale einer $\mathrm{Cu}_{3} \mathrm{Si}: \mathrm{Ni}$ - und einer $\mathrm{NiSi}_{2}$ :Cu-Ausscheidung überlappen, wie im Zusammenhang mit Ausscheidung P2 in Abb. 4.12 gezeigt wurde. Der niedrigste Messwert des partiellen Molenbruch in dieser Untersuchung ist vergleichbar dem $X_{C u}^{(p)}$-Wert der isolierten $\mathrm{NiSi}_{2}$ :Cu-Ausscheidung P3 in Abb. 4.13 und Abb. 4.9. 
Die STEM-Aufnahme in Abb. 4.15 zeigt einen repräsentativen Abschnitt der Kolonie, für deren Ausscheidungen in Abb. 4.14 die Häufigkeitsverteilung des partiellen Molenbruchs $X_{C u}^{(p)}$ dargestellt ist. Der mittlere Abstand zweier Ausscheidungen innerhalb dieser Kolonie lässt sich mit $74 \pm 9 \mathrm{~nm}$ abschätzen. Dabei sind die Ausscheidungen nicht gleichmäßig verteilt sondern entlang gekrümmter Linien aufgereiht, was sich konsistent mit dem Wachstumsmechanismus für Kolonien durch Bildung und Wachstum von $\mathrm{Cu}_{3} \mathrm{Si}$-Ausscheidungen an einer kletternden Versetzung erklären lässt. Die $\mathrm{NiSi}_{2}: \mathrm{Cu}-$ Ausscheidungen an den markierten Stellen sind in gleicher Weise angeordnet und unterscheiden sich auch in ihrer Größe nicht von $\mathrm{Cu}_{3} \mathrm{Si}$ :Ni- Ausscheidungen. 


\subsubsection{Ausscheidungen unter nickelreichen Bedingungen}

Um Ausscheidungen zu untersuchen, die sich nach Kodiffusion von Kupfer und Nickel unter nickelreichen Bedingungen nach Gleichung 4.1 definiert als $Q_{C u, N i}<1$ bilden, wurde bei einem weiteren Kodiffusionsexperiment die Randbedingung für die Diffusion von Kupfer so modifiziert, dass sich für Kupfer ein Konzentrationsprofil bei gleichzeitig fester Konzentration von Nickel einstellte (Probe 4, Tab. B.1 in Anhang B.1).

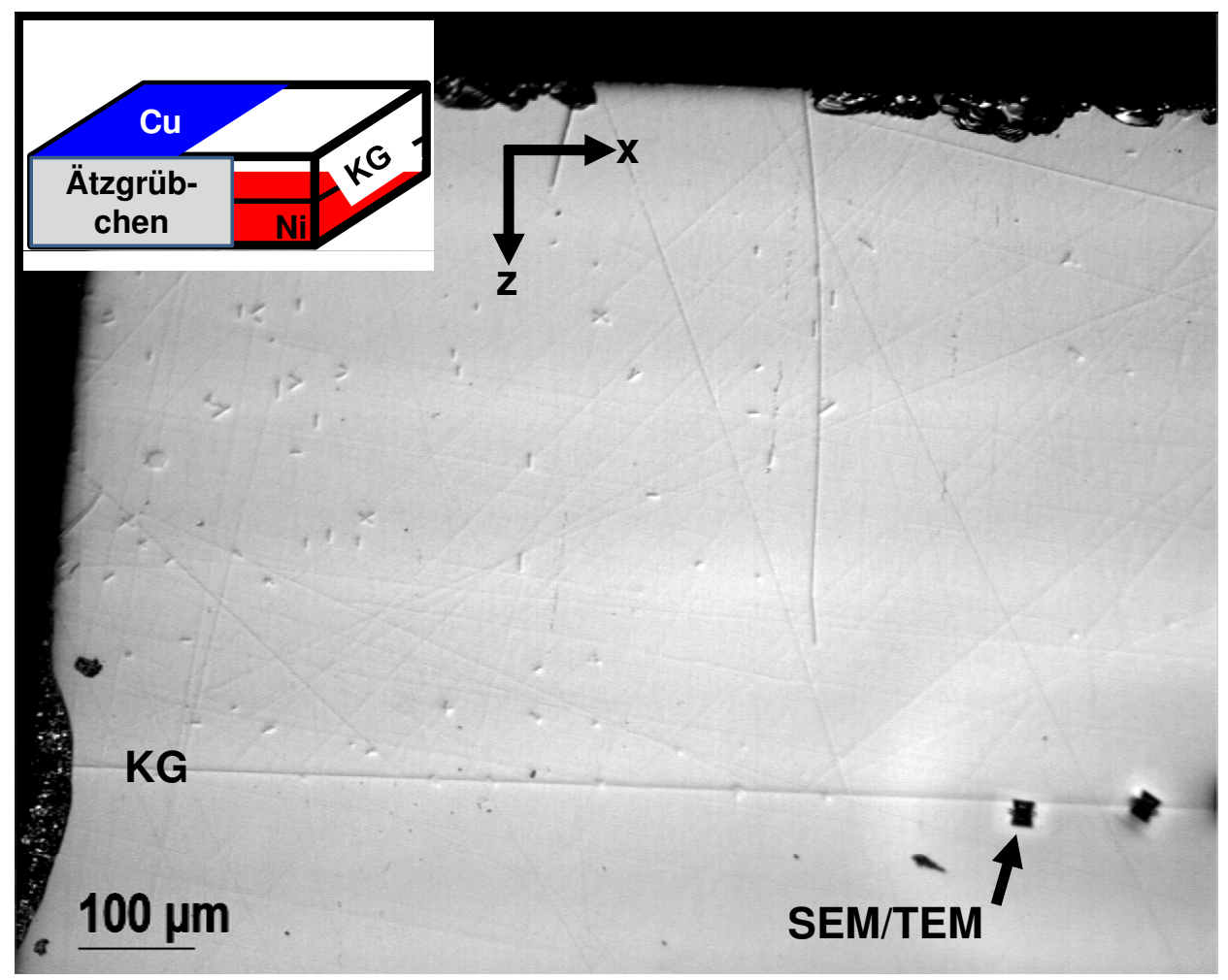

Abbildung 4.16. - Lichtmikroskopische Aufnahme eines Querschnitts durch eine bikristalline Probe nach inhomogener Kodiffusion von $\mathrm{Cu}$ und $\mathrm{Ni}$ bei $900^{\circ} \mathrm{C}$ und 90 s Diffusionsdauer, langsamer Abkühlung und Defektätzung (Secco, 5s). Die eingefügte Zeichnung zeigt schematisch die Lage des abgebildeten Probenbereiches, die Anordnung der als Diffusionsquellen für $\mathrm{Cu}$ und Ni dienenden Schichten und die Korngrenze (KG). An der durch einen Pfeil gekennzeichneten Stelle wurden weitere Untersuchungen mit SEM und TEM durchgeführt.

Die genaue Geometrie dieses Experimentes ist in Abb. 4.16 dargestellt. Nach halbseitiger Kupferbedeckung einer Probenoberfläche und ganzseitiger Nickelbedeckung der anderen Oberfläche und Kodiffusion von $\mathrm{Cu}$ und Ni bei $900^{\circ} \mathrm{C}$ und $90 \mathrm{~s}$ Dauer ist zu 
erwarten, dass sich in $\mathrm{x}$-Richtung für $\mathrm{Cu}$ ein abfallendes Konzentrationsprofil bei fester Nickelkonzentration ergibt. In Übereinstimmung mit dieser Erwartung zeigt die in Abb. 4.16 dargestellte Ätzgrübchenuntersuchung, dass die Dichte von Ätzgrübchen mit der für Kolonien aus $\mathrm{Cu}_{3} \mathrm{Si}$-Ausscheidungen typischen Morphologie in x-Richtung abnimmt.

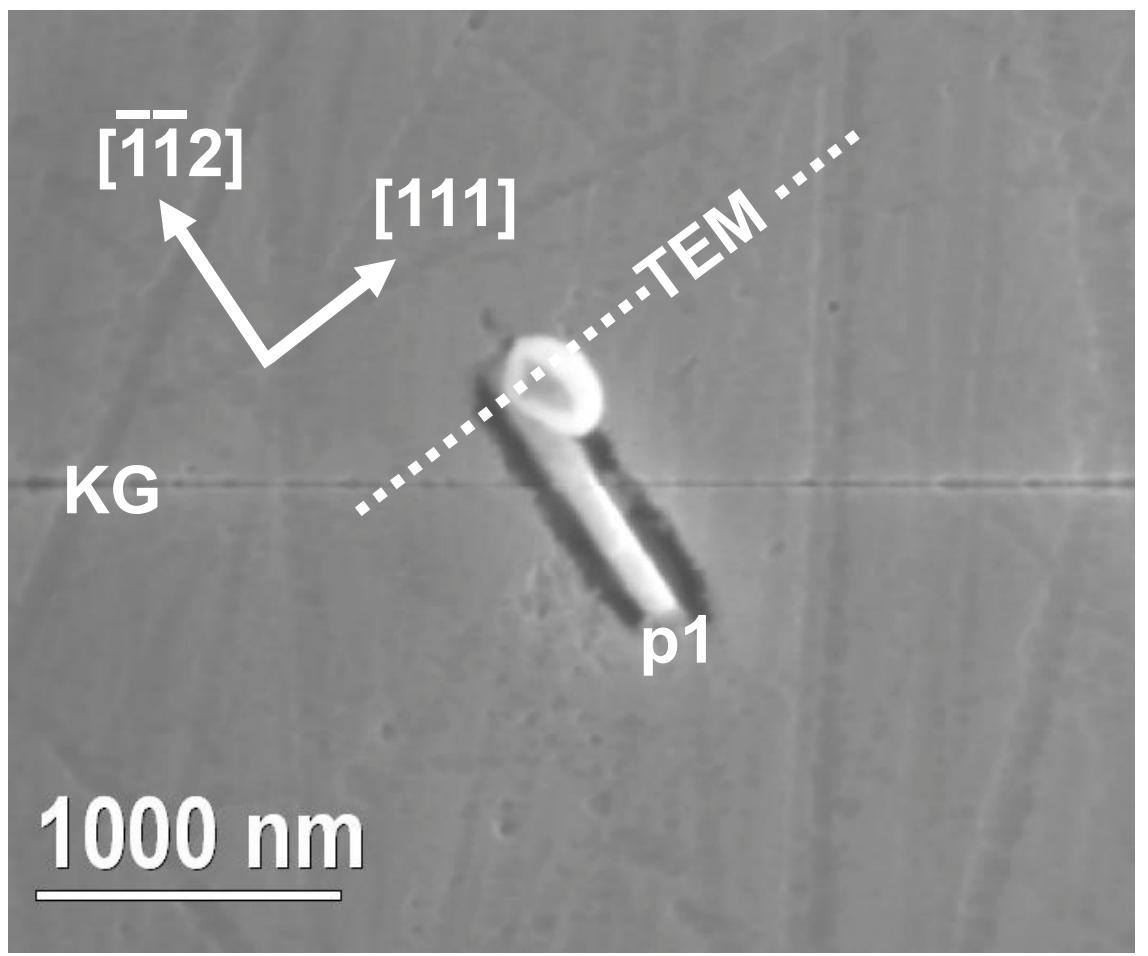

Abbildung 4.17. - SEM-Aufnahme eines einzelnen Ätzgrübchens (p1) an der Korngrenze (KG) einer bikristallinen Probe nach Kodiffusion von $\mathrm{Cu}$ und $\mathrm{Ni}\left(900^{\circ} \mathrm{C}\right.$, 90s) unter nickelreichen Bedingungen, langsamer Abkühlung und Defektätzung (Secco, 5s). Der planare Defekt (p1) innerhalb des Ätzgrübchens ist in Abb. 4.18 mit TEM im Querschnitt dargestellt.

Ätzgrübchen, die innerhalb eines Bereiches der Probe liegen, in dem die Kupferkonzentration so gering ist, dass keine Ätzgrübchen mit der für Kolonien von $\mathrm{Cu}_{3} \mathrm{Si}$ Ausscheidungen typischen Form mehr erkennbar sind, weisen auf Ausscheidungen hin, die sich unter nickelreichen Bedingungen gebildet haben. Abb. 4.17 stellt eine rasterelektronenmikroskopische (SEM) Aufnahme eines solchen Ätzgrübchens in Aufsicht dar. Das Ätzgrübchen liegt an der in Abb. 4.16 mit einem Pfeil markierten Stelle direkt an der Korngrenze. Es hat eine längliche Form, die auf einen planaren Defekt (p1) parallel 
zu einer (111)-Ebene hinweist. Mit einem fokussierten Ionenstrahl (FIB) war es möglich, entlang der unterbrochen gezeichneten Linie eine elektronentransparente Lamelle für TEM-Untersuchungen mit [ $\overline{11} 2]$-orientierter Oberfläche zu präparieren.

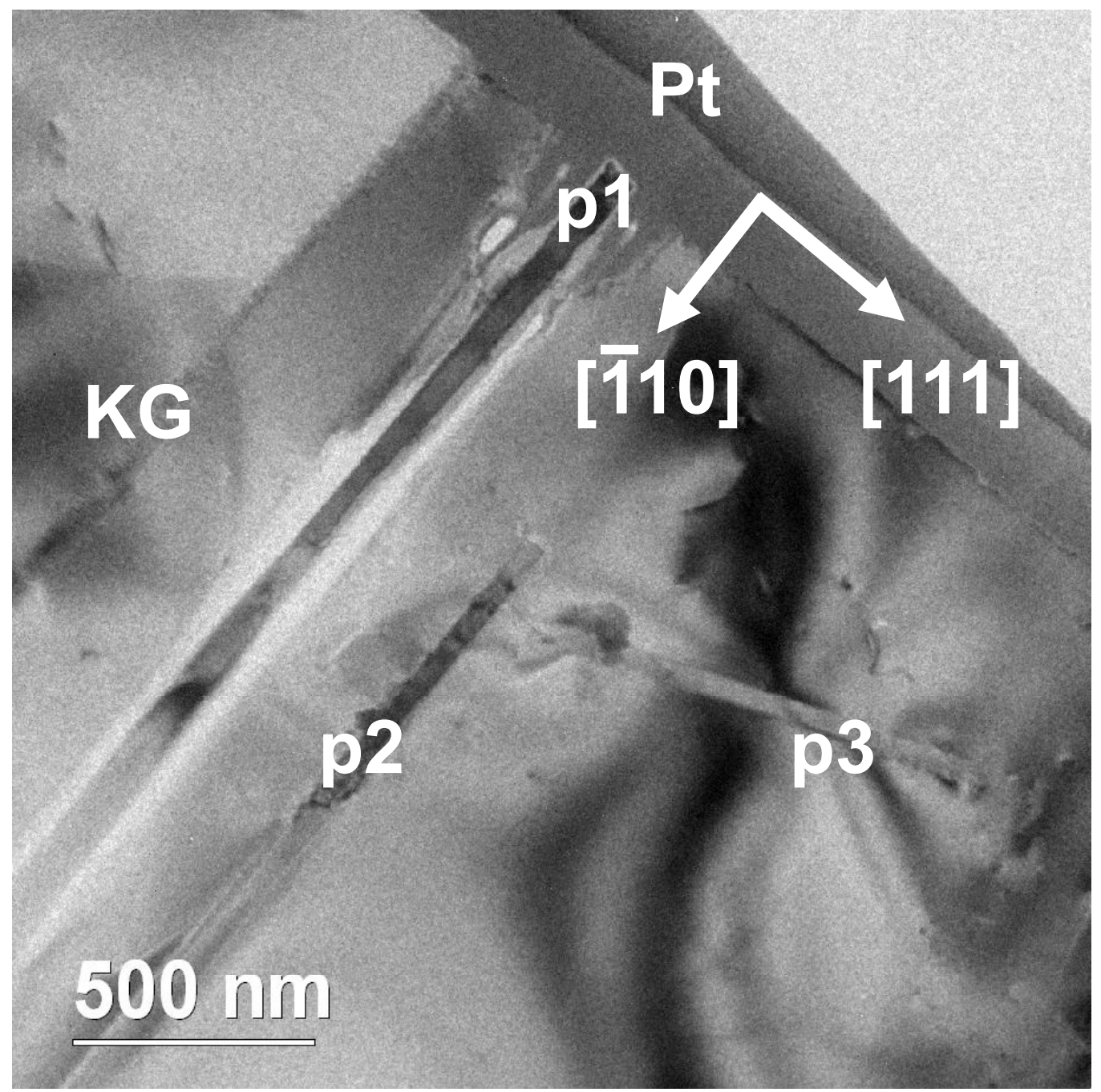

Abbildung 4.18. - TEM-Aufnahme eines plättchenförmigen Teilchens (p1) innerhalb des in Abb. 4.17 gezeigten Ätzgrübchens an der Korngrenze (KG) einer bikristallinen Probe nach Kodiffusion von $\mathrm{Cu}$ und $\mathrm{Ni}\left(900^{\circ} \mathrm{C}, 90 \mathrm{~s}\right)$ unter nickelreichen Bedingungen, langsamer Abkühlung und Defektätzung (Secco, 5s). Weitere Teilchen (p2 und p3) liegen unterhalb der mit Platin bedeckten (Pt) Probenoberfläche. 
Die TEM-Aufnahme in Abb. 4.18 stellt einen Querschnitt durch die in Abb. 4.17 gezeigte Probenstelle mit einem Ätzgrübchen an der Korngrenze dar. Die Probenoberfläche ist infolge der FIB-Präparation mit einer ca. $200 \mathrm{~nm}$ dicken Pt-Schicht (Pt) bedeckt. Bei Vergleich von Abb. 4.17 und Abb. 4.18 stellt sich heraus, dass der als Ursache für die Bildung des Ätzgrübchens vermutete planare Defekt ein plättchenförmiges Teilchen (p1) ist, das auf einer (111)-Ebene angesiedelt ist. Zwei weitere plättchenförmige Teilchen (p2, p3) unterhalb der Probenoberfläche liegen auf $\{111\}$-Ebenen. Die Teilchen sind ungefähr gleich groß bei einem Durchmesser von einem Mikrometer und eine Dicke von $50 \mathrm{~nm}$. Plättchen dieser Art stellen eine typische Defektstruktur von Ausscheidungen von $\mathrm{NiSi}_{2}$ nach langsamer Abkühlung dar.

Die in Abb. 4.19 dargestellten Punktmessungen von EDX-Spektren an den plättchenförmigen Teilchen zeigen, dass es sich um Ausscheidungen bestehend aus $\mathrm{Si}, \mathrm{Ni}$ und $\mathrm{Cu}$ handelt. Die zusätzlich auftretende $\mathrm{Mo}_{L \alpha}$-Linie ist auf den Probenhalter zurückzuführen. Die genaue Zusammensetzung der Ausscheidung lässt sich durch Quantifizierung der Spektren nach der Methode von Cliff und Lorimer bestimmen [78]. Bei Teilchen dieser Größe liefert die Si-Matrix anders als im Fall der Kolonien kleinerer Teilchen, die unter kupferreichen Bedingungen beobachtet werden, keinen Beitrag mehr zum Spektrum. Der Si-Anteil der Ausscheidungen kann somit exakt bestimmt werden. Es zeigt sich, dass die Ausscheidungen aus Ni und Si in einem Verhältnis von ungefähr 1:2 bestehen und dabei einen Anteil von durchschnittlich 4 At.\% Cu enthalten. Die großen Plättchen lassen sich somit wie die kleineren polyedrischen Teilchen, die nach Kodiffusion unter kupferreichen Bedingungen vorliegen, vgl. Abschnitt 4.2.1.3, als Ausscheidungen der $\mathrm{NiSi}_{2}$ : $\mathrm{Cu}$-Phase identifizieren.

\begin{tabular}{|c|c|c|c||c|}
\hline Ausscheidung & \multicolumn{3}{|c||}{ Molenbruch (At.\%) } & partieller Molenbruch (\%) \\
& $\mathrm{Cu}$ & $\mathrm{Ni}$ & $\mathrm{Si}$ & $\mathrm{Cu}$ \\
\hline $\mathrm{p} 1$ & $4.3 \pm 0.5$ & $33.0 \pm 0.5$ & $62.7 \pm 1.0$ & $11.5 \pm 1.2$ \\
$\mathrm{p} 2$ & $3.9 \pm 0.5$ & $30.4 \pm 0.5$ & $65.7 \pm 1.0$ & $11.4 \pm 1.2$ \\
$\mathrm{p} 3$ & $4.1 \pm 0.8$ & $31.9 \pm 1.1$ & $64.0 \pm 2.1$ & $11.4 \pm 2.0$ \\
\hline
\end{tabular}

Tabelle 4.4. - Zusammensetzung plättchenförmiger Ausscheidungen nach Kodiffusion von $\mathrm{Cu}$ und $\mathrm{Ni}$ $\left(900^{\circ} \mathrm{C}, 90 \mathrm{~s}\right)$ unter nickelreichen Bedingungen und langsamer Abkühlung. Die Angaben beruhen auf der quantitativen Analyse der in Abb.4.19 gezeigten EDX-Spektren.

Tabelle 4.4 stellt die Werte für die Zusammensetzung der $\mathrm{NiSi}_{2}: \mathrm{Cu}$-Ausscheidungen unter nickelreichen Bedingungen bei $900^{\circ} \mathrm{C}$ zusammen. Bei der Abweichung der Zusammensetzung der Teilchen von der exakten Stöchiometrie für reines $\mathrm{NiSi}_{2}$ ergibt sich ein uneinheitliches Bild:

Die Ausscheidung p1 enthält den gleichen Anteil Ni wie binäres NiSi 2 . Die zusätzlich enthaltene Menge Cu führt zu einer Verringerung des Si-Anteils. Die Ausscheidung p2 


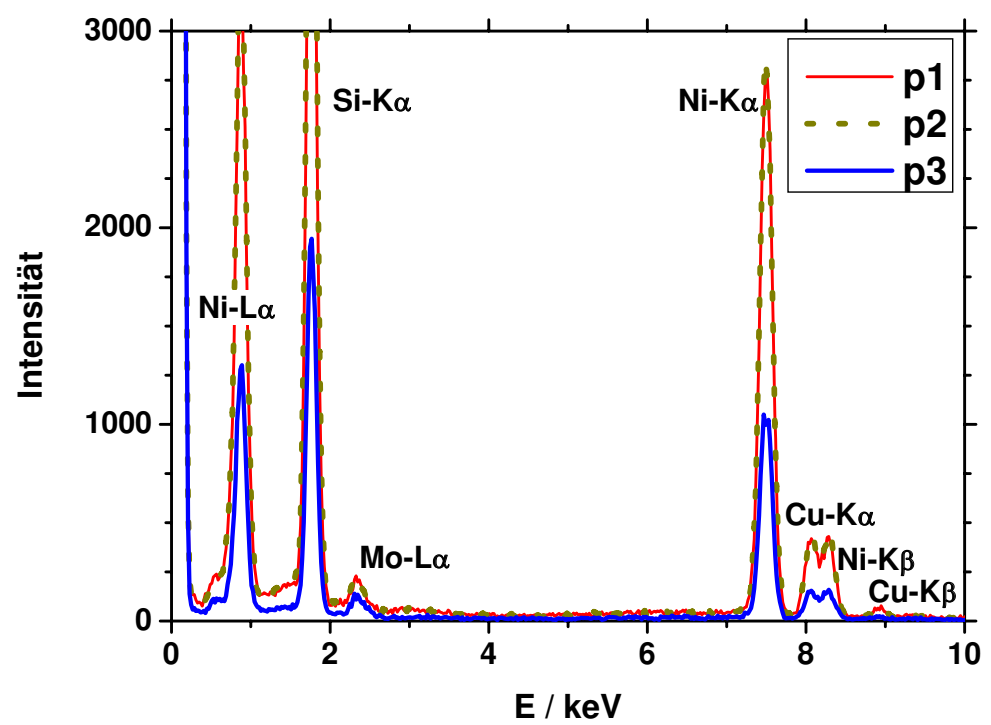

(a) EDX-Spektren

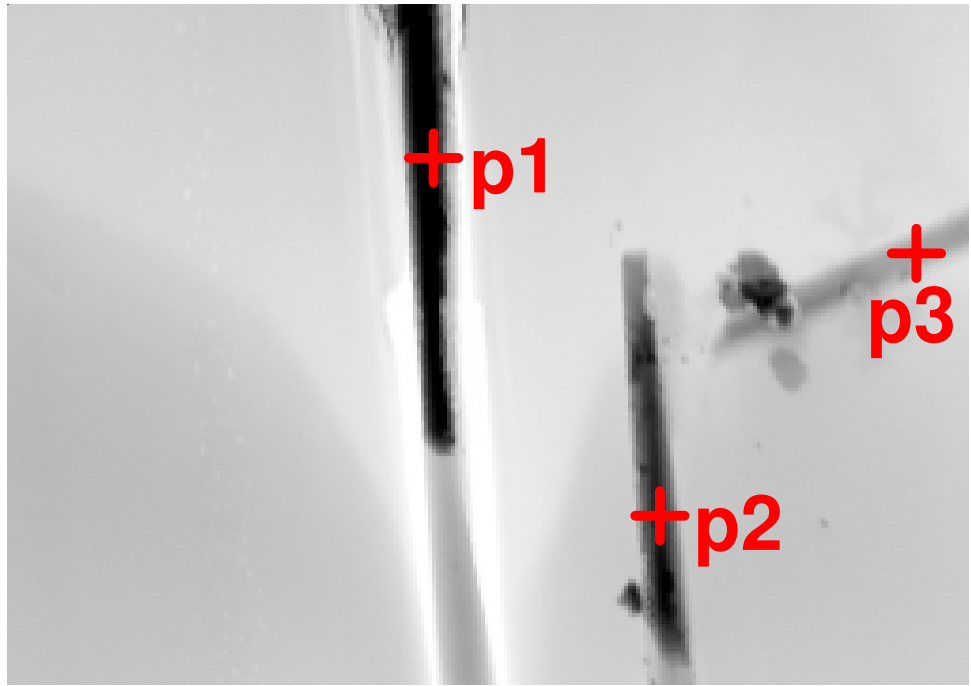

(b) STEM

Abbildung 4.19. - (a) EDX-Spektren und (b) STEM-Aufnahme plättchenförmiger Ausscheidungen in Si nach Ko-Diffusion von $\mathrm{Cu}$ und $\mathrm{Ni}\left(900^{\circ} \mathrm{C}, 90 \mathrm{~s}\right)$ unter Ni-reichen Bedingungen und langsamer Abkühlung. Die quantitative Analyse der Spektren zeigt, dass die Ausscheidungen aus Ni und Si im Verhältnis 1:2 und zusätzlich einem Anteil von durchschnittlich $4 \mathrm{At} . \% \mathrm{Cu}$ bestehen.

hingegen enthält den gleichen Anteil $\mathrm{Si}$ wie binäres $\mathrm{NiSi}_{2}$. Die zusätzliche Menge $\mathrm{Cu}$ 
führt hier zu einer Verringerung des Ni-Anteils. In der Ausscheidung p3 schließlich führt das zusätzlich vorhandene $\mathrm{Cu}$ sowohl zu einer Verringerung des Si- wie des Ni-Anteils.

Außer der genauen Zusammensetzung ist in Tab. 4.4 auch der partielle Molenbruch von $\mathrm{Cu}$ angegeben. Die Werte sind niedriger als die der $\mathrm{NiSi}_{2}: \mathrm{Cu}$-Ausscheidungen unter kupferreichen Bedingungen bei $1050^{\circ} \mathrm{C}$, vgl. Tabelle 4.3 .

Die großen plättchenförmigen Ausscheidungen werden von kleineren kugelförmigen Teilchen dekoriert, wie in den STEM-Aufnahmen der Abb. 4.19 und Abb. 4.20 zu sehen ist. EDX-Spektren nach Punktmessungen an diesen Teilchen, dargestellt in Abb. 4.20, zeigen außer der $\mathrm{Si}_{K \alpha}$-Linie und für $\mathrm{Cu}$ charakteristischen Linien auch in geringer Intensität die $\mathrm{Ni}_{K \alpha}$-Linie. Die Teilchen lassen sich daher als Ausscheidungen einer Kupfersilizid-Phase interpretieren. Der Ni-Anteil des in Abb. 4.20 mit k1 bezeichneten Teilchens kann durch einen partiellen Molenbruch von $X_{N i}^{(p)}=1.9 \pm 1.3 \%$ ausgedrückt werden. Für das andere Teilchen (k2) ist der Beitrag der $K_{\alpha}$-Linie von Ni zum Spektrum nicht signifikant. Die in beiden Spektren auftretenden L-Linien von Iod lassen sich auf eine unbeabsichtigte Kontamination der Probe in der FIB-Kammer zurückführen. 


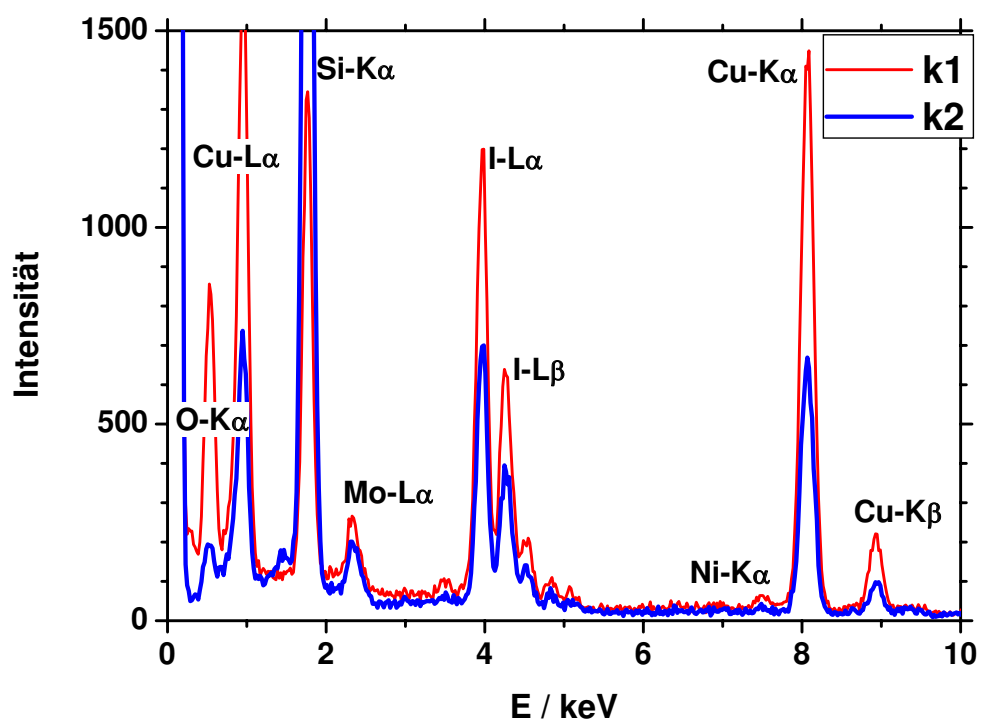

(a) EDX-Spektren



(b) STEM

Abbildung 4.20. - (a) EDX-spektren und (b) STEM-Aufnahme kleinerer Ausscheidungen einer Kupfersilizid-Phase am Rand plättchenförmiger $\mathrm{NiSi}_{2}: \mathrm{Cu}$-Ausscheidungen nach Kodiffusion von $\mathrm{Cu}$ und $\mathrm{Ni}\left(900^{\circ} \mathrm{C}\right.$, 90s) unter nickelreichen Bedingungen und langsamer Abkühlung. 


\subsubsection{Zusammenfassung}

Es lässt sich sowohl unter kupfer- als auch unter nickelreichen Bedingungen die Bildung von Ausscheidungen zweier Phasen beobachten, die jeweils beide metallische Verunreinigungen enthalten.

Bei der einen Phase $\left(\mathrm{Cu}_{3} \mathrm{Si}: \mathrm{Ni}\right)$ handelt es sich um eine Lösung von $\mathrm{Ni}$ in $\mathrm{Cu}_{3} \mathrm{Si}$. Ausscheidungen dieser Phase bilden Kolonien, zeigen eine starke Wechselwirkung mit Versetzungen und unterscheiden sich durch abweichende Gitterparameter strukturell von $\mathrm{Si}$, wie es für Ausscheidungen von $\mathrm{Cu}_{3} \mathrm{Si}$ der Fall ist.

Die andere Phase ist $\mathrm{NiSi}_{2}$ : $\mathrm{Cu}$, es handelt sich um eine Lösung von $\mathrm{Cu}$ in $\mathrm{NiSi}_{2}$. Ausscheidungen dieser Phase zeigen die gleichen strukturellen Merkmale wie reine $\mathrm{NiSi}_{2}$ Ausscheidungen: polyedrische und plättchenförmige Morphologie, $\mathrm{CaF}_{2}$-Struktur und das Auftreten von Zwillingen. Im Fall nickelreicher Bedingungen lässt sich die Zusammensetzung $\mathrm{NiSi}_{2}$ durch EDX-Messungen direkt nachweisen.

Form, Verteilung und Größe der Ausscheidungen nach Kodiffusion von $\mathrm{Cu}$ und $\mathrm{Ni}$ in Si und langsamer Abkühlung hängen vom Konzentrationsverhältnis beider metallischer Verunreinigungen ab. Es dominiert eine der beiden Mikrostrukturen, die bei der Ausscheidung von $\mathrm{Cu}$ oder $\mathrm{Ni}$ als alleiniger metallischer Verunreinigung in Si beobachtet wird.

Unter kupferreichen Bedingungen $Q_{C u, N i}>1$ kommt es zur Bildung von Kolonien kleiner Teilchen, die Ausscheidungen zweier verschiedener Phasen sind, $\mathrm{Cu}_{3} \mathrm{Si}: \mathrm{Ni}$ und $\mathrm{NiSi}_{2}: \mathrm{Cu}$. Unter nickelreichen Bedingungen $Q_{C u, N i}>1$ dagegen bilden sich große plättchenförmige Ausscheidungen $\left(\mathrm{NiSi}_{2}: \mathrm{Cu}\right)$, die von kleineren Teilchen einer anderen Phase dekoriert sind. 


\subsection{Ausscheidungen nach Kodiffusion von Palladium und Nickel}

Bei $T=1050{ }^{\circ} \mathrm{C}, \dot{T}=-6 \mathrm{~K} / \mathrm{s}$ und in bikristallinem Probenmaterial wurden Ausscheidungen zweier metallischer Verunreinigungen durch Kodiffusion und anschließende langsame Abkühlung präpariert. (Proben 6 und 7, Tab. B.1 in Anhang B.1). In diesem Experiment wurden Palladium und Nickel als metallische Verunreinigungen ausgewählt. Palladium zeigt als alleinige metallische Verunreinigung in Si ein mit Kupfer vergleichbares Ausscheidungsverhalten, da $\mathrm{Pd}_{2}$ Si eine ähnlich hohe Volumenfehlpassung (110\%) besitzt wie $\mathrm{Cu}_{3} \mathrm{Si}$ [25]. Da Palladium in Silizium eine geringere Löslichkeit besitzt als Nickel, vgl. Tabelle 4.1, liegen in der Probe gemäß Gleichung 4.1 nickelreiche Bedingungen vor: $Q_{N i, P d}>1$, so dass zu erwarten ist, dass Form, Größe und Verteilung der Ausscheidungen nach Kodiffusion von Palladium und Nickel durch niedrige Volumenfehlpassung bestimmt ist.

\subsubsection{Verteilung}



Abbildung 4.21. - Lichtmikroskopische Querschnittsaufnahme einer bikristallinen Probe nach Diffusion von Pd bei $1050^{\circ} \mathrm{C}$, langsamer Abkühlung und Defektätzung (Secco, 6s). Ätzgrübchen zeigen die Lage der Korngrenze (KG) und zahlreicher ausgedehnter Defekte an der Korngrenze und im Inneren einer der Scheiben (B) an. 


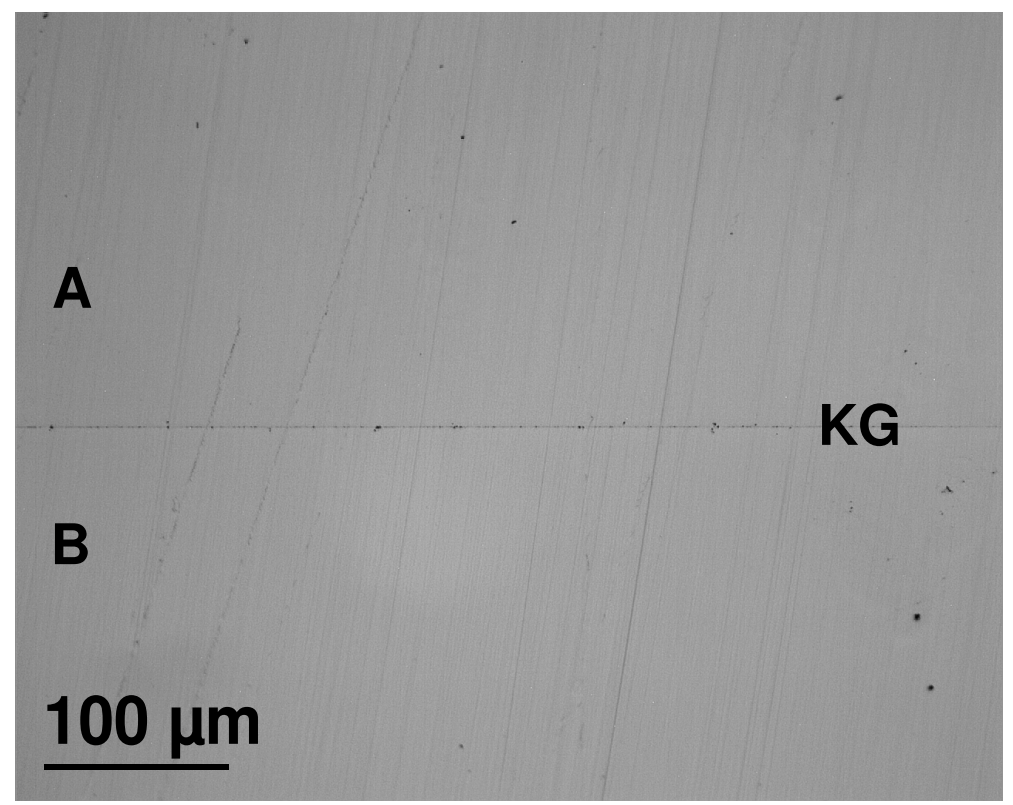

Abbildung 4.22. - Lichtmikroskopische Querschnittsaufnahme einer bikristallinen Probe nach Kodiffusion von $\mathrm{Pd}$ und $\mathrm{Ni}$ bei $1050^{\circ} \mathrm{C}$, langsamer Abkühlung und Defektätzung (Secco, 6s). Ätzgrübchen zeigen die Lage der Korngrenze (KG) und einiger weniger ausgedehnter Defekte an der Korngrenze zwischen den Scheiben $(A, B)$ an.

Palladium scheidet sich als alleinige metallische Verunreinigung in Si ein wie Kupfer in Form von Kolonien aus. Parallel zu den Experimenten mit $\mathrm{Cu}$ als Einzelverunreinigung wurde in der vorliegenden Arbeit eine Probe des Bikristalls auch mit Pd durch Diffusion unter den gleichen Prozessbedingungen $\left(T=1050^{\circ} \mathrm{C}, \dot{T}=-6 \mathrm{~K} / \mathrm{s}\right)$ gezielt verunreinigt (Probe 5, Tab.B.1 in Anhang B.1). Nach Defektätzen zeigt sich eine inhomogene Verteilung kleiner Ätzgrübchen, vgl. Abb. 4.21. Sie weist auf das Vorliegen von ausgedehnten Defekten an der Korngrenze und in Scheibe B hin, in der kleine Oxidausscheidungen und Versetzungsringe präsent sind, siehe Anhang A. Die Verteilung der Defekte ist somit qualitativ mit der nach Diffusion von $\mathrm{Cu}$ oder Kodiffusion von $\mathrm{Cu}$ und Ni unter kupferreichen Bedingungen vergleichbar, vgl. Abb. 4.1 und Abb. 4.2 in Abschnitt 4.1 und Abb. 4.3 in Abschnitt 4.2.1.1.

Das Ergebnis einer Ätzgrübchenuntersuchung, dargestellt in Abb. 4.22, nach Kodiffusion von Palladium und Nickel, zeigt eine ganz andere Defektverteilung. Insgesamt enthält die Probe nur wenige Defekte, die vorwiegend an der Korngrenze liegen. Das Volumen der Probe auf beiden Seiten der Korngrenze ist praktisch defektfrei. Die weitere Untersuchung zeigt, dass es sich bei den an der Korngrenze liegenden Defekten um einzelne Ausscheidungen der $\mathrm{NiSi}_{2}: \mathrm{Pd}-\mathrm{Ph}$ ase handelt. Diese Ausscheidungen stellen 


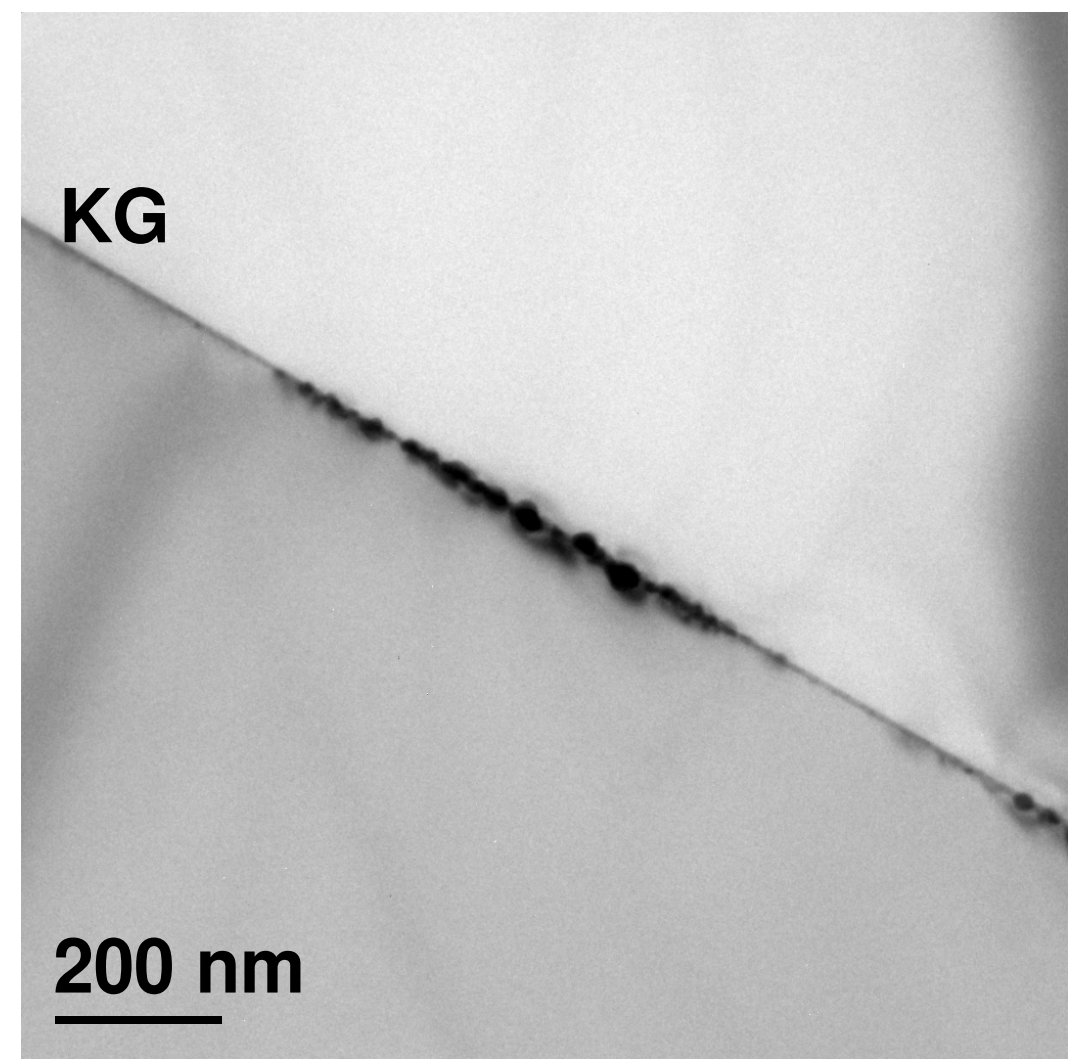

Abbildung 4.23. - TEM-Hellfeldaufnahme einer Kolonie kleiner Palladiumsilizidausscheidungen an der Korngrenze (KG) einer bikristallinen Probe nach Diffusion von Pd bei $1050^{\circ} \mathrm{C}$ und langsamer Abkühlung.

unter den Bedingungen der langsamen Abkühlung effektive Diffusionssenken für $\mathrm{Pd}$ Atome dar, da die für die Ausscheidung von Pd typische Bildung von Kolonien kleinerer Ausscheidungen nicht stattfindet.

\subsubsection{Struktur}

Als alleinige metallische Verunreinigung scheidet sich Pd in Form von Kolonien aus. Abb. 4.23 zeigt eine solche Kolonie an der Korngrenze einer mit $\mathrm{Pd}$ bei $1050^{\circ} \mathrm{C}$ verunreinigten Probe des Bikristalls.

Nach Kodiffusion von Pd und Ni in bikristallinem Si und langsamer Abkühlung lassen sich an der Korngrenze mit TEM polyedrische Teilchen finden, deren Seitenflächen vorwiegend parallel zu $\{111\}$-Ebenen liegen, wie Abb. 4.24 zeigt. Diese Teilchen stellen 


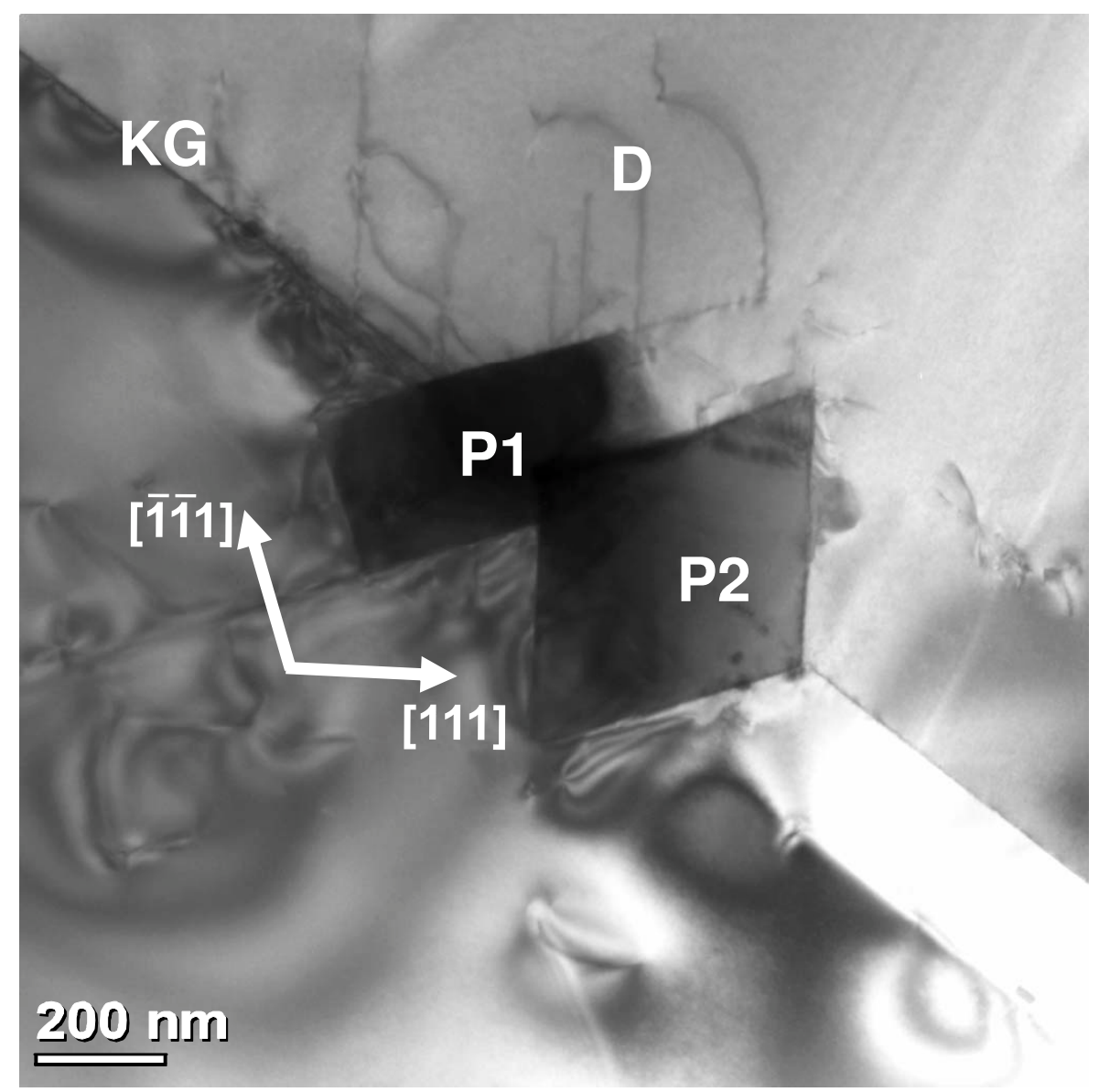

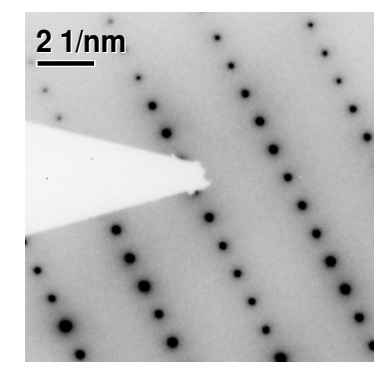

(b) Ausscheidung P1

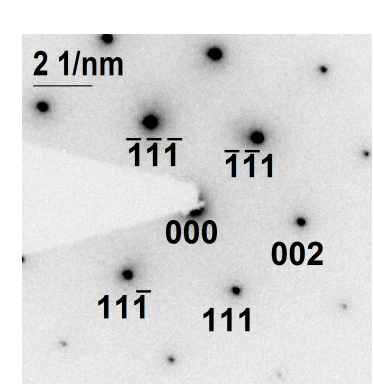

(c) $\mathrm{Si}$

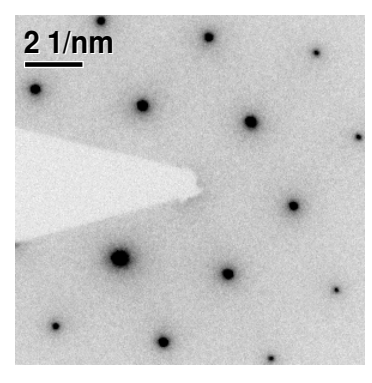

(d) Ausscheidung P2

Abbildung 4.24. - (a) TEM-Hellfeldaufnahme polyedrischer Ausscheidungen an der Korngrenze (KG) einer bikristallinen Probe nach Kodiffusion von Pd und $\mathrm{Ni}$ bei $1050^{\circ} \mathrm{C}$ und langsamer Abkühlung. Die Ausscheidungen (P1, P2) sind von Versetzungen (D) dekoriert. (b)-(d) Feinbereichsbeugungsbilder zeigen, dass die Ausscheidungen eine fcc-Kristallstruktur und nahezu diegleiche Gitterkonstante haben wie Si. Die Ausscheidung P1 weist eine $Z$ willingsorientierung bezüglich der Matrix auf. 
sich als Ausscheidungen einer binären Phase aus Ni und Si dar, in der außerdem Pd gelöst enthalten ist, wie die weiteren Untersuchungen zeigen werden.

Das ebenfalls in Abb. 4.24 enthaltene Feinbereichsbeugungsbild der Ausscheidung P2 weist dieselben Beugungsreflexe auf wie das der Si-Matrix. Dagegen befinden sich im Beugungsbild der Ausscheidung P1 zusätzliche Reflexe, die sich durch das Vorliegen ei-

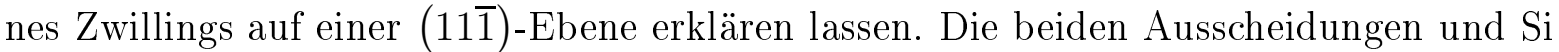
haben kubisch-flächenzentrierte Kristallstrukturen mit nahezu der gleichen Gitterkonstanten wie $\mathrm{Si}$, so wie es für $\mathrm{NiSi}_{2}$ mit der kubischen $\mathrm{CaF}_{2}$-Struktur der Fall ist. Dabei weist die Zwillingsorientierung von $\mathrm{P} 1$ auf eine $\mathrm{NiSi}_{2}$-Ausscheidung vom Typ B hin, während die Ausscheidung P2 vom Typ A ist.

Die TEM-Aufnahme zeigt viele Versetzungen, die von den Ausscheidungen ausgestoßen werden. Dies lässt sich durch die Gitterfehlpassung der ausgeschiedenen Phase gegenüber Si erklären. Zwar ist die Fehlpassung von $\mathrm{NiSi}_{2}$ sehr klein, es ist jedoch bekannt, dass auch eine geringe relative Volumenfehlpassung zwischen Ausscheidung und Matrix bei großen Ausscheidungen zu einer Verzerrung führt, die durch plastische Verformung der Ausscheidung oder Erzeugung von Versetzungen wieder abgebaut wird [79].

In der Umgebung der großen polyedrischen Ausscheidungen sowie an deren Grenzfläche zur Si-Matrix befinden sich außerdem kleinere Teilchen, bei denen es sich um Ausscheidungen einer weiteren ternären Phase aus Pd, Ni und Si handeln könnte. Abb. 4.25 stellt eine dieser kleineren Ausscheidungen (hier mit K bezeichnet) mit hochauflösender TEM dar. Die Fouriertransformierte der Gitterabbildung des Teilchens enthält zusätzliche Intensitätsmaxima im Vergleich zur Fouriertransformierten der Si-Matrix. Dies zeigt, dass die kleineren Ausscheidungen sich im Gegensatz zu den großen polyedrischen Ausscheidungen strukturell von Si unterscheiden. 


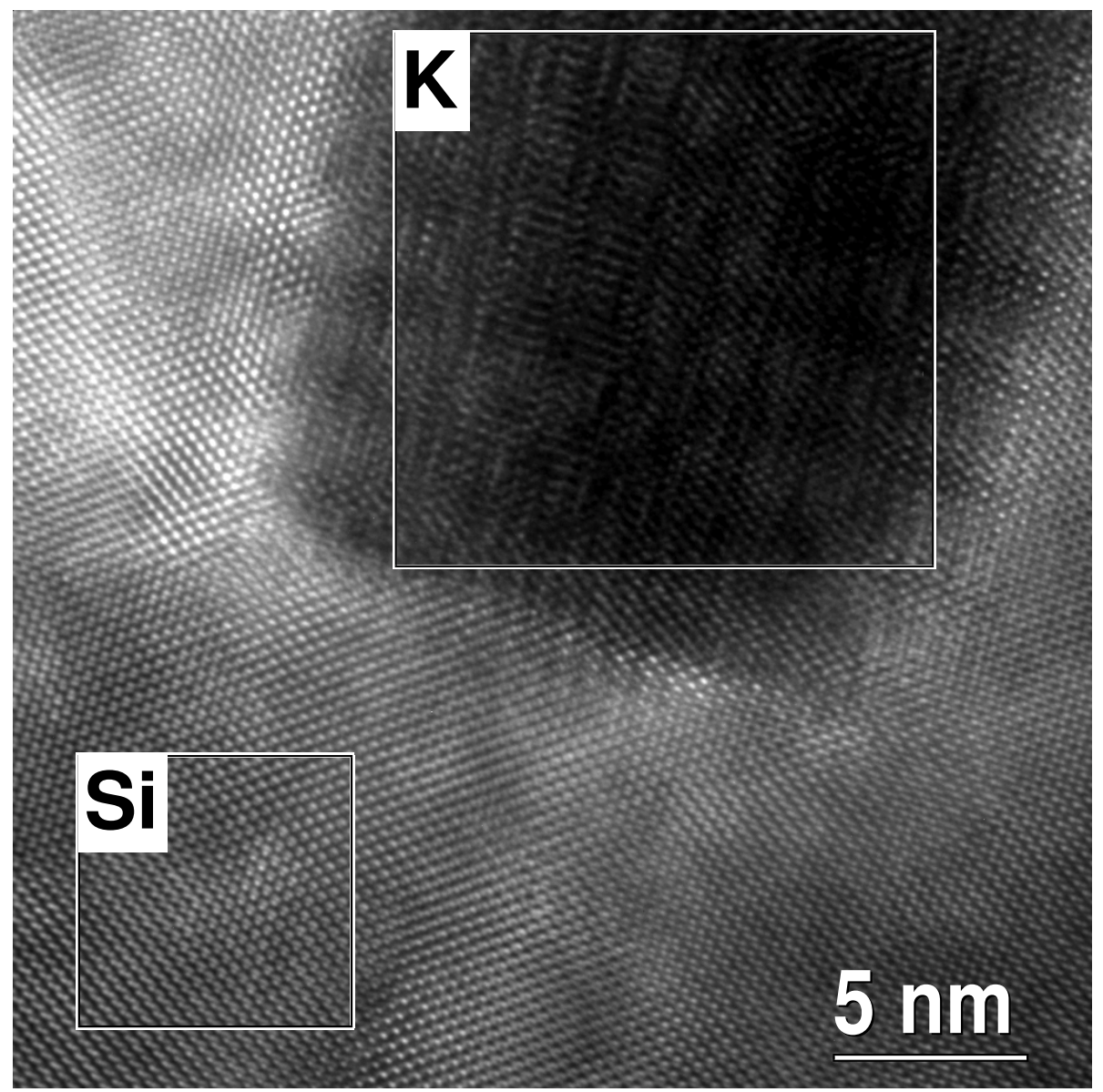

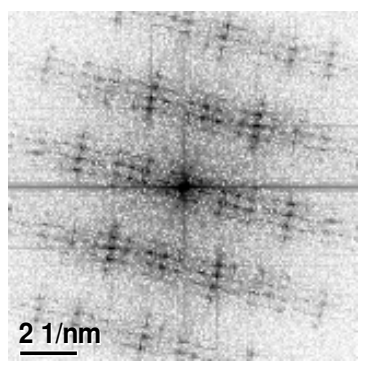

(b)



(c)

Abbildung 4.25. - (a) Hochaufösende TEM-Aufnahme einer kleineren Ausscheidung nach Kodiffusion von $\mathrm{Pd}$ und Ni bei $1050^{\circ} \mathrm{C}$ und langsamer Abkühlung. (b) Das Diffraktogramm des eingerahmten Bereiches innerhalb der Ausscheidung (K) zeigt zusätzliche Intensitätsmaxima verglichen mit (c) dem Diffraktogramm eines Bereiches der Si-Matrix, was auf unterschiedliche Gitterparameter der Kristallstrukturen hinweist. 


\subsubsection{Zusammensetzung}

Nach Kodiffusion von $\mathrm{Pd}$ und Ni in bikristallinem Si und langsamer Abkühlung lässt sich die Bildung großer polyedrischer an der Korngrenze liegender Teilchen beobachten. Abb. 4.26 zeigt ein EDX-Spektrum aus einer Punktmessung an einem der beiden polyedrischen Teilchen, die im vorangegangenen Abschnitt 4.3.2 Gegenstand der Betrachtungen waren. Die Teilchen lassen sich als Ausscheidungen identifizieren, in denen beide metallische Verunreinigungen ( $\mathrm{Ni}$ und $\mathrm{Pd}$ ) gleichzeitig vorliegen. Bei EDX-Messungen an Teilchen, deren Größe die Probendicke übertrifft, wie es für die Ausscheidung P in Abb. 4.26 der Fall ist, liefert die Si-Matrix keinen Beitrag zum EDX-Spektrum mehr. Daher lässt sich durch quantitative Analyse der Spektren nach Cliff und Lorimer [78] zeigen, dass die Ausscheidungen aus Ni und Si im Verhältnis 1:2 bestehen und einen kleinen Anteil von 1.4 At.\% Pd enthalten. Die genauen Werte der Zusammensetzung sind in Tabelle 4.5 angegeben. Die Phase der Ausscheidungen lässt sich als Lösung von $\mathrm{Pd}$ in $\mathrm{NiSi}_{2}$ interpretieren und wird im Folgenden mit $\mathrm{NiSi}_{2}: \mathrm{Pd}$ bezeichnet.

Abb. 4.27 zeigt EDX-Karten der in Abb. 4.24 dargestellten Probenstelle. Ein starkes $\mathrm{Ni}_{K \alpha}$-Signal bildet die Lage und die Konturen der $\mathrm{NiSi}_{2}: \mathrm{Pd}-$ Ausscheidungen ab. Ein schwaches homogenes $\mathrm{Pd}_{L \alpha}$-Signal innerhalb der polyedrischen Ausscheidungen zeigt, dass das in den Ausscheidungen eingebaute Pd gleichmäßig verteilt ist. Die kleineren Teilchen, die am Rand oder in der Umgebung der polyedrischen $\mathrm{NiSi}_{2}$-Teilchen liegen, zeigen ein $\mathrm{Pd}_{L \alpha}$-Signal, das wesentlich höher ist als innerhalb der $\mathrm{NiSi}_{2}$-Ausscheidungen. Sie lassen sich als Ausscheidungen einer Palladium-Silizid-Phase beschreiben.

Abb. 4.28 enthält eine EDX-Messung und eine STEM-Aufnahme einer weiteren polyedrischen $\mathrm{NiSi}_{2}: \mathrm{Pd}$-Ausscheidung an einer anderen Probenstelle. Die $\mathrm{NiSi}_{2}: \mathrm{Pd}-\mathrm{Ausschei}-$ dung $(\mathrm{P})$ wird von einer kleineren Ausscheidung $(\mathrm{K})$ der Palladium-Silizid-Phase dekoriert. Bei der EDX-Messung wurden beide Ausscheidungen entlang der in der STEMAufnahme markierten Strecke mit dem Elektronenstrahl abgetastet. Die Messung der Intensität der $\mathrm{Ni}_{K \alpha^{-}}$und der Pd-L $\alpha$-Linie zeigt, dass die Palladium-Silizid-Ausscheidung einen geringen Anteil Ni enthält und somit ebenfalls aus einer ternären Phase besteht. EDX-Punktmessungen zur näheren Bestimmung der Zusammensetzung wurden ebenfalls durchgeführt, die Ergebnisse sind in Tabelle 4.5 und Tabelle 4.6 aufgeführt. Im Fall der Palladium-Silizid-Ausscheidung, die klein gegenüber der Probendicke ist, wird der partielle Molenbruch von Pd als Maß für die Zusammensetzung benutzt.

Nach Kodiffusion von $\mathrm{Pd}$ und $\mathrm{Ni}$ in einer einkristallinen Probe und langsamer Abkühlung lassen sich an der Oberfläche der Probe große polyedrische Ausscheidungen beobachten, die von kleineren Partikeln dekoriert sind, wie die TEM-Aufnahme in Abb. 4.29 demonstriert. Quantitative Analysen von EDX-Spektren nach Punktmessungen innerhalb der großen polyedrischen $(\mathrm{P})$ und der kleineren Ausscheidung (K) in Abb. 4.29 


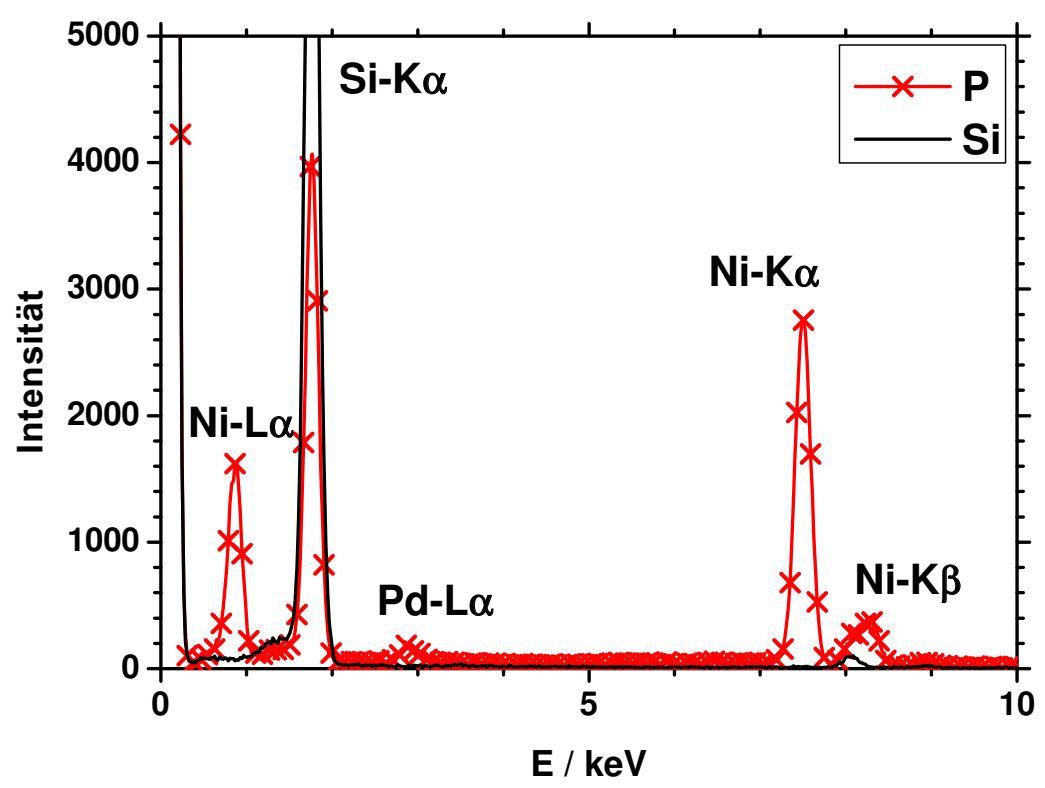

(a) EDX-Spektren

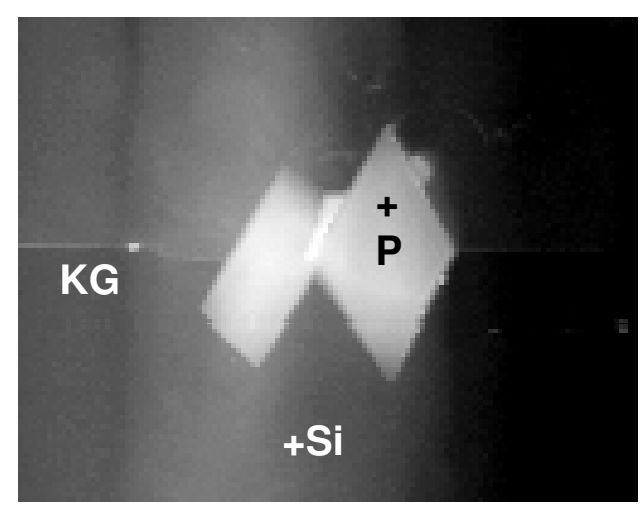

(b) STEM

Abbildung 4.26. - (a) EDX-Spektrum und (b) STEM-Aufnahme einer der in Abb. 4.24 gezeigten polyedrischen Ausscheidungen an der Korngrenze $(K G)$ in einer bikristallinen Probe nach Kodiffusion von Pd und Ni bei $1050^{\circ} \mathrm{C}$ und langsamer Abkühlung. Die quantitative Analyse des Spektrums zeigt, dass die Ausscheidung $(P)$ im Verhältnis 1:2 aus Ni und Si und einem zusätzlichen Anteil von 1.4 At.\% Pd besteht. 


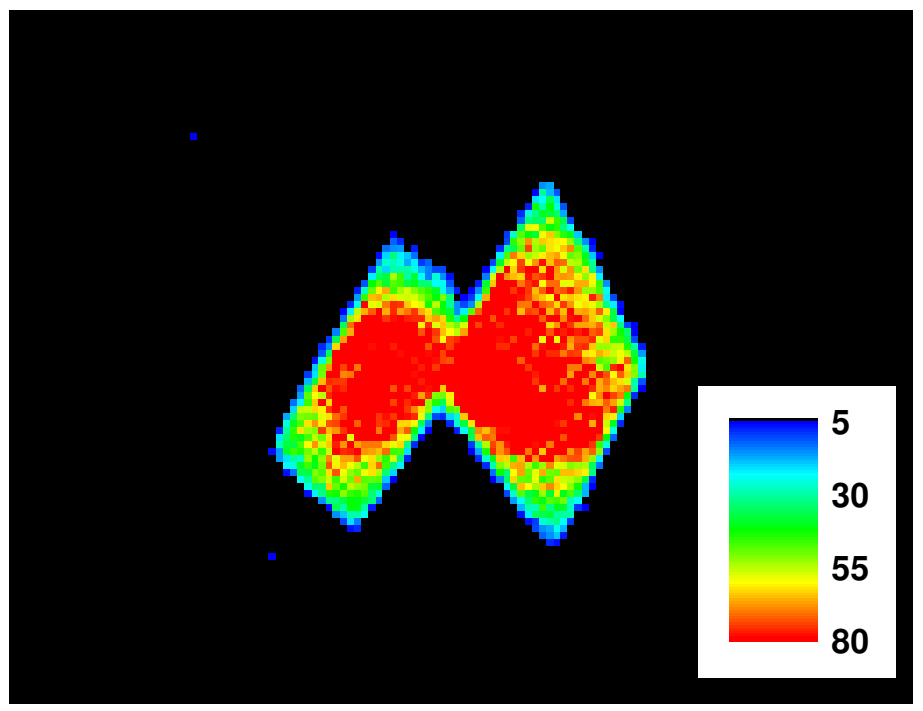

(a) $\operatorname{EDX~} \mathrm{Ni}_{K \alpha}$

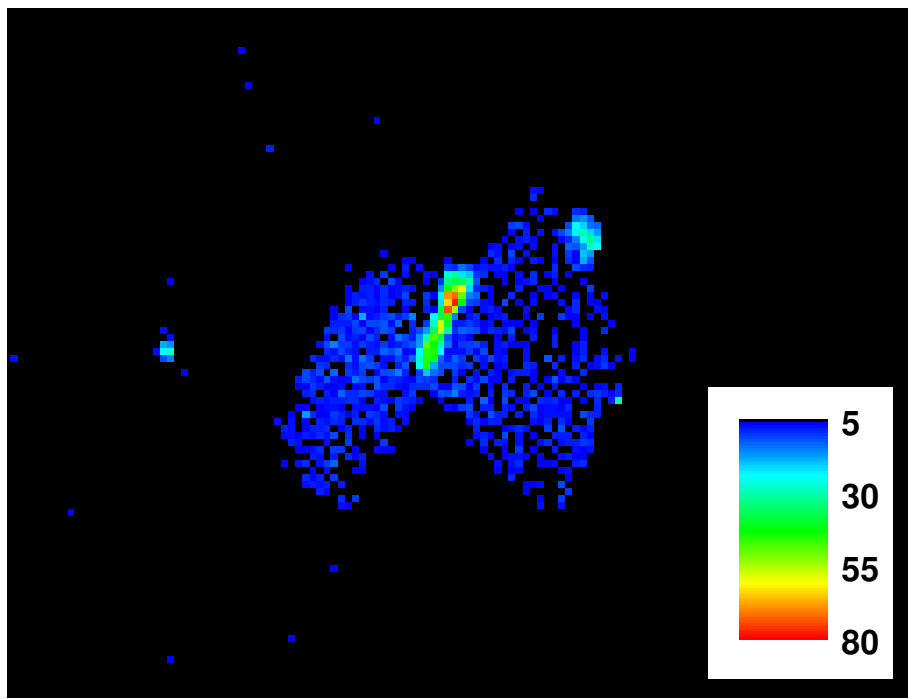

(b) EDX $\operatorname{Pd}_{L \alpha}$

Abbildung 4.27. - EDX-Karten der in Abb.4.24 gezeigten polyedrischen $\mathrm{NiSi}_{2}: P d$-Ausscheidungen nach Kodiffusion von $\mathrm{Pd}$ und $\mathrm{Ni}$ bei $1050^{\circ} \mathrm{C}$ und langsamer Abkühlung. Die laterale Verteilung der Intensität (a) der $\mathrm{Ni}_{K \alpha}$ und (b) $P d_{L \alpha}$-Röntgenstrahlung zeigt, dass die $\mathrm{NiSi}_{2}: \mathrm{Pd}$-Ausscheidungen von kleineren palladiumreichen Ausscheidungen dekoriert werden. 




(a) EDX

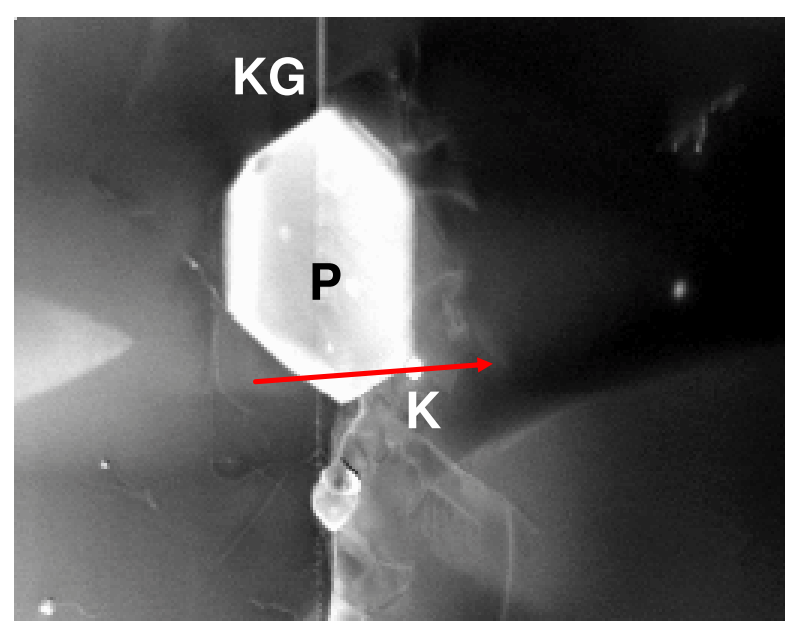

(b) STEM

Abbildung 4.28. - (a) EDX-Messung an und (b) STEM-Aufnahme von Ausscheidungen an der Korngrenze $(K G)$ in einer bikristallinen Probe nach Kodiffusion von Pd und Ni bei $1050^{\circ} \mathrm{C}$ und langsamer Abkühlung. Dargestellt sind die unkorrigierten Intensitäten der Si- und $\mathrm{Ni}_{K \alpha^{-}}$, sowie der $P d_{L \alpha}$-Röntgenstrahlung. Die polyedrische $\mathrm{NiSi}_{2}: \mathrm{Pd}$ Ausscheidung $(\mathrm{P})$ enthält Pd und die Palladium-Silizid-Ausscheidung (K) einen kleinen Anteil Ni. 




(a) TEM

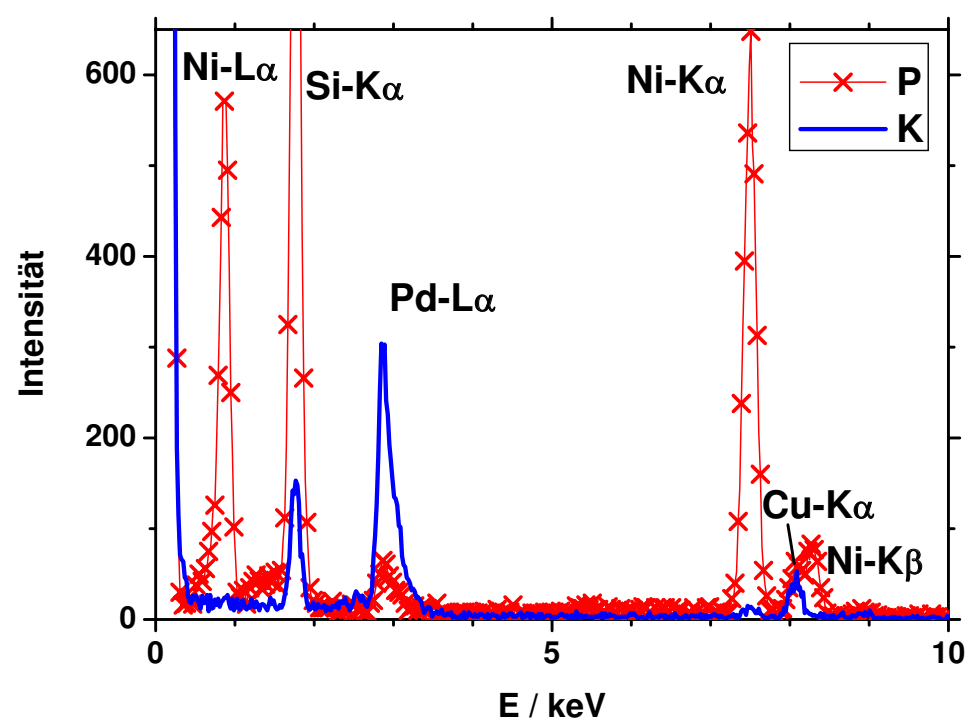

(b) EDX-Spektren

Abbildung 4.29. - (a) TEM-Aufnahme und (b) EDX-Spektren von Ausscheidungen an der Oberfläche einer einkristallinen Probe nach Kodiffusion von $P d$ und Ni bei $1050^{\circ} \mathrm{C}$ und langsamer Abkühlung. Die große Ausscheidung $(P)$ besteht aus der NiSi $: P d-$ Phase und die kleine Ausscheidung (K) aus einer Palladium-Silizidphase mit Ni. An der Probenoberfläche befinden sich Überreste der als Diffusionsquelle verwendeten Pd-Schicht $(P d)$. 


\begin{tabular}{|c|c|c|c|c|}
\hline EDX-Messung & \multicolumn{3}{|c|}{ Molenbruch (At.\%) } & partieller Molenbruch (\%) \\
& $\mathrm{Pd}$ & $\mathrm{Ni}$ & $\mathrm{Si}$ & $\mathrm{Pd}$ \\
\hline Abb. 4.26 & $1.4 \pm 0.2$ & $34.4 \pm 0.5$ & $64.1 \pm 0.5$ & $4.0 \pm 0.5$ \\
Abb. 4.28 & $1.3 \pm 0.2$ & $33.7 \pm 0.4$ & $65.0 \pm 0.4$ & $3.8 \pm 0.5$ \\
Abb. 4.29 & $2.6 \pm 0.4$ & $30.8 \pm 1.0$ & $66.5 \pm 1.0$ & $7.9 \pm 1.1$ \\
\hline
\end{tabular}

Tabelle 4.5. - Zusammensetzung von $\mathrm{NiSi}_{2}: \mathrm{Pd}$-Ausscheidungen nach Kodiffusion von Pd und Ni bei $1050^{\circ} \mathrm{C}$ und langsamer Abkühlung. Die Angaben beruhen auf der quantitativen Analyse von EDX-Spektren nach Punktmessungen an der in der jeweiligen Messung mit $P$ bezeichneten Ausscheidung.

\begin{tabular}{|c|c|c|c|c|}
\hline EDX-Messung & \multicolumn{3}{|c|}{ Molenbruch (At.\%) } & partieller Molenbruch (\%) \\
& $\mathrm{Pd}$ & $\mathrm{Ni}$ & $\mathrm{Si}$ & $\mathrm{Pd}$ \\
\hline Abb. 4.28 & \multicolumn{2}{|c|}{ keine Angabe möglich } & $93.9 \pm 1.6$ \\
Abb. 4.29 & $60.7 \pm 1.7$ & $2.3 \pm 1.2$ & $36.9 \pm 1.7$ & $96.3 \pm 1.8$ \\
\hline
\end{tabular}

Tabelle 4.6. - Zusammensetzung von Ausscheidungen der Palladium-Silizid-Phase nach Kodiffusion von $\mathrm{Pd}$ und $\mathrm{Ni}$ bei $1050^{\circ} \mathrm{C}$ und langsamer Abkühlung. Die Angaben beruhen auf der quantitativen Analyse von EDX-Spektren nach Punktmessungen an der in der jeweiligen Messung mit $K$ bezeichneten Ausscheidung. Im Fall der Messung in Abb. 4.28 kann die genaue Zusammensetzung nicht angegeben werden, da die Ausscheidung klein im Vergleich zur Probendicke ist.

ergeben ähnliche Zusammensetzungen wie bei entsprechenden Ausscheidungen, die in bikristallinen Proben an der Korngrenze gefunden wurden. Da bei dieser Probe die untersuchte Stelle nahe am Probenrand liegt, ist die Probe hinreichend dünn, um aus der Messung die Zusammensetzung auch des kleineren Teilchens erhalten zu können. Die genauen Werte sind in Tab. 4.6 enthalten. Es ergibt sich ein Pd:Si-Verhältnis von ungefähr 2:1, so dass die Palladium-Silizid-Phase als Lösung von $\mathrm{Ni}$ in $\mathrm{Pd}_{2} \mathrm{Si}$ interpretiert werden kann. Die in den Spektren zusätzlich auftretende $\mathrm{Cu}_{K \alpha}$-Linie ist auf das TEM-Netzchen aus $\mathrm{Cu}$ zurückzuführen.

Die Tabellen 4.5 und 4.6 fassen die Werte für die Zusammensetzung der untersuchten Ausscheidungen nach Kodiffusion von Pd und Ni zusammen. Der partielle Molenbruch von $\mathrm{Pd}$ in Ausscheidungen der $\mathrm{NiSi}_{2}$ :Pd-Phase beträgt etwas 4 bis $8 \%$. Die Zusammensetzung der Palladium-Silizid-Teilchen ist vergleichbar der von $\mathrm{Pd}_{2} \mathrm{Si}$. Ausscheidungen dieser Phase enthalten einen kleinen Anteil Ni, der ausgedrückt werden kann durch einen Wert von $95 \%$ für den partiellen Molenbruch von Pd. 
$\Gamma_{\text {Kapitel }}$

\section{Zusammenfassung und Diskussion}

\subsection{Verteilung von Metallsilizidausscheidungen im Bikristall}

\subsubsection{Zusammenfassung}

Die vorliegende Arbeit untersuchte zunächst die Verteilung von Metallsilizidausscheidungen bei gleichzeitiger Anwesenheit einer Korngrenze und von Mikrodefekten. Als Probenmaterial diente ein Bikristall bestehend aus zwei durch eine Kleinwinkelkorngrenze miteinander verbundene Scheiben aus Cz-Si. Die Korngrenze lässt sich als Netzwerk aus Stufen- und Schraubenversetzungen beschreiben, wobei der Abstand zwischen den Stufenversetzungen $372 \mathrm{~nm}$ und der zwischen den Schraubenversetzungen $12 \mathrm{~nm}$ beträgt. Eine der Scheiben ist nahezu defektfrei, während das Volumen der anderen Scheibe eine hohe Dichte von Mikrodefekten enthält. Bei ihnen handelt es sich um Siliziumoxidausscheidungen und Versetzungen. Die Mikrostruktur der Proben stellt damit ein Modell für die Mikrostruktur multikristallinen Siliziums dar, das als Ausgangsmaterial für Solarzellen verwendet wird.

Nach Verunreinigung mit Kupfer als alleiniger metallischer Spezies durch Diffusion bei $T_{C u}=1050^{\circ} \mathrm{C}$ wurden während langsamer Abkühlung mit einer Rate von $\dot{T}=6 \mathrm{~K} / \mathrm{s} \mathrm{Ko-}$ lonien kleinerer Ausscheidungen präpariert. Es ist bekannt [14, 25, 42 44], dass es sich bei den Ausscheidungen selber um Teilchen mit einer Größe von einigen Nanometern bis bis zu einigen zehn Nanometern handelt, während die Kolonien Größen von bis zu 80 $\mu \mathrm{m}$ haben.

Die räumliche Verteilung der von den Ausscheidungen gebildeten Kolonien wurde durch Defektätzen bestimmt. Sie ist inhomogen, wie die in Abschnitt 4.1 dargestellten lichtmikroskopischen Aufnahmen zeigen. Eine hohe Dichte der Kolonien ließ sich an der Korngrenze und in der Scheibe beobachten, in der auch die Dichte der Mikrodefekte 
hoch ist. In der nahezu defektfreien Scheibe liegen dagegen fast keine Kolonien vor. In der Scheibe mit vielen Mikrodefekten (Scheibe B) liegt unterhalb der Oberfläche der Probe eine ausscheidungsfreie Zone vor, wenn diese Oberfläche mit einer Schicht bestehend aus der binären Cu-Si-Phase bedeckt ist, mit der Si nach dem Phasendiagramm im Gleichgewicht steht. Eine der Probenoberflächen ist mit einer Schicht aus dieser binären $\mathrm{Cu}$-Si-Phase bedeckt, die während der Diffusion als Quelle für die Verunreinigung der Probe mit $\mathrm{Cu}$ dient, während die andere Oberfläche frei ist. Die ausscheidungsfreie Zone in Scheibe B tritt nicht auf, wenn die Oberfläche auf dieser Seite der Probe frei ist.

\subsubsection{Diskussion}

Die Bildung von Ausscheidungen einer metallischen Verunreinigung $M$ in einem binären System M-Si setzt eine Übersättigung voraus,

$$
s=\frac{[M]}{[M]_{s o l}}>1,
$$

also das Vorliegen einer Konzentration $[M]$ oberhalb der Löslichkeit $[M]_{\text {sol }}$ von $M$ in Si. Diese Situation wurde experimentell durch die Abkühlphase nach der Eindiffusion der metallischen Verunreinigung bei hoher Temperatur realisiert. Im Fall heterogener Keimbildung ist die für die Ausscheidungsbildung notwendige kritische Übersättigung $s_{\text {het }}$ geringer als bei homogener Keimbildung, $s_{\text {het }}<s_{\text {hom }}$, so dass bei Vorhandensein entsprechender Keimbildungsplätze heterogene Keimbildung zuerst einsetzt. Nach der Keimbildung findet eine Umverteilung der metallischen Fremdatome in die wachsenden Ausscheidungen statt, so dass die Konzentration der Fremdatome in Si verringert wird. Bei hinreichend hohem Diffusionskoeffizienten der Fremdatome verringert sich deren Konzentration rascher als die Löslichkeit, so dass die für homogene Keimbildung nötige kritische Übersättigung $s_{\text {hom }}$ niemals erreicht wird. In ähnlicher Weise können auch verschiedene Arten heterogener Keimbildungsplätze mit unterschiedlichen kritischen Übersättigungen $s_{1}, s_{2}, .$. miteinander konkurrieren.

Es ist bekannt, dass Kupfer als metallische Verunreinigung in Si zur Bildung von Ausscheidungen an strukturellen Defekten wie beispielsweise Versetzungen neigt [80]. Die Ursache dafür liegt in der Wechselwirkung zwischen Versetzungen und interstiellen Si-Atomen, die aufgrund der Volumenfehlpassung zwischen Si und der ausgeschiedenen Phase erzeugt werden können [25, 42]. Daher lässt sich die in dieser Arbeit beobachtete hohe Dichte von Kolonien kleinerer Kupfersilizidausscheidungen in der mit B bezeichneten Scheibe mit heterogener Keimbildung an den in dieser Scheibe vorhandenen Siliziumoxidausscheidungen und Versetzungen erklären.

In der Probe insgesamt stellen die Mikrodefekte in Scheibe B dabei keineswegs die einzigen Plätze für heterogene Keimbildung von Ausscheidungen dar. Vielmehr konkurrieren 
sie wie oben für heterogene und homogene Keimbildung beschrieben mit Keimbildung an der Kleinwinkelkorngrenze zwischen Scheibe A und Scheibe B, der freien Oberfläche und möglicherweise in Scheibe A in einer geringen Dichte vorliegenden Mikrodefekten. Außerdem konkurrieren die Ausscheidungen bei der Umverteilung der Cu-Atome mit der als Diffusionsquelle verwendeten Schicht aus einer binären $\mathrm{Cu}-\mathrm{Si}$-Gleichgewichtsphase an einer der Probenoberflächen. Die Auswirkung der konkurrierenden Keimbildungs- und Umverteilungsprozesse auf die räumliche Verteilung der Kolonien zeigt sich am Vorhandensein ausscheidungsfreier Zonen, auf deren Vorhandensein im Zusammenhang mit der $\mathrm{Cu}-\mathrm{Si}$-Phase an der Oberfläche bereits hingewiesen wurde.

An der Oberfläche stellt sich im Gleichgewicht mit der Cu-Si-Phase die Löslichkeit ein, d.h. es gilt $s=1$ als Randbedingung. Sobald die Temperatur sinkt und im Volumen eine Übersättigung entsteht, besteht eine treibende Kraft für die Umverteilung von CuAtomen aus dem Volumen der Probe in die Cu-Si-Phase auf der Oberfläche.

Die heterogene Keimbildung an Mikrodefekten $(\mu D)$ im Volumen setzt erst bei einer kritischen Übersättigung $s_{\mu D}>1$ ein. Bei Konkurrenz mit der Cu-Si-Phase an der Oberfläche resultiert ein Bereich, in dem die Konzentration auch bei weiterer Abkühlung nicht mehr ausreicht, um Ausscheidungen an Mikrodefekten zu bilden, so dass sich wie in Abschnitt 4.1 beobachtet eine ausscheidungsfreie Zone ergibt.

Das Fehlen einer ausscheidungsfreien Zone an der Korngrenze zeigt, dass sich die Konzentration der metallischen Verunreinigung im Si durch Keimbildung und Ausscheidungswachstum an der Korngrenze nicht schneller verringert als durch die Mikrodefekte. Die Reduzierung der Konzentration durch Ausscheidung hängt nicht nur vom Eintreten der Keimbildung, sondern auch von der Kinetik des Ausscheidungsvorgangs ab, d.h. vom Diffusionskoeffizient der Fremdatome und der Volumendichte der Ausscheidungen. Dabei spielt auch die Dichte der Keimbildungsplätze eine Rolle. Eine Einschätzung der Höhe der kritischen Übersättigung für Keimbildung an der Korngrenze im Vergleich mit den Mikrodefekten ist an dieser Stelle nicht möglich.

Untersuchungen zum Ausscheidungsverhalten schnell diffundierender metallischer Verunreinigung ( $\mathrm{Co}, \mathrm{Ni}, \mathrm{Cu}$ und $\mathrm{Pd}$ ) in defektfreiem Si haben gezeigt, dass bei einer Abkühlrate von $4 \mathrm{~K} / \mathrm{s}$ die Bildung der Ausscheidungen nahezu vollständig an der freien Oberfläche stattfindet und homogene Keimbildung im Volumen der Probe deutlich übertrifft, [25, 26]. Unter diesen Bedingungen weisen die freien Oberflächen von Proben nach Defektätzen eine hohe Dichte von Ätzgrübchen auf, was als „haze“ bezeichnet wird [81]. Mikrodefekte wie Versetzungen und Siliziumoxidausscheidungen relativieren den Einfluss der freien Oberfläche als Platz für heterogene Keimbildung, wie Graff et al. mit Hilfe von Palladium als „Test-Verunreinigung“ demonstrierten [38] und durch die vorliegende Arbeit bestätigt wird.

Die Charakterisierung von Solarzellen aus multikristallinem Silizium (mc-Si) mit Me- 
thoden, die ortsaufgelöst die Lebensdauer der durch Lichteinfall erzeugten Minoritätsladungsträger oder den Photostrom (LBIC: „Ligh-Beam-Induced-Current") messen, zeigt, dass einzelne Körner aus mc-Si in ihren elektrischen Eigenschaften sehr unterschiedlich sein können. Betrachtet man den in dieser Arbeit verwendeten Si-Bikristall als ein Modell für zwei durch eine Kleinwinkelkorngrenze verbundene Körner in realem mc-Si, wird deutlich, dass die Verteilung einer metallischen Verunreinigung und damit die elektrischen Eigenschaften im Wesentlichen durch die Verteilung von Mikrodefekten innerhalb der Körner wie Siliziumoxidausscheidungen und Versetzungen bestimmt wird. Teile der in dieser Arbeit mit $\mathrm{Cu}$ als alleiniger metallischer Verunreinigung sowie mit $\mathrm{Cu}$ und $\mathrm{Ni}$ gemeinsam verunreinigten Bikristalle wurden mit LBIC untersucht, [82] und zeigen eine deutlich erhöhte Rekombinationsaktivität in der Scheibe, die viele Mikrodefekte enthält.

Die ausscheidungsfreie Zone in Anwesenheit von Mikrodefekten demonstriert, dass eine auf der gesamten Scheibenoberfläche aufgebrachte Metallsilizidphase in der Lage ist, effektiv metallische Verunreinigungen aus der Scheibe zu entfernen. Eine entsprechende Beobachtung für Ni als metallische Verunreinigung in einkristallinem defektfreiem Silizium machten Gay und Martinuzzi, die diesen Vorgang als „self-gettering“ bezeichnen [83]. Diese Arbeit zeigt, dass die Umverteilung metallischer Verunreinigungen in eine Randphase auch bei Präsenz von Mikrodefekten, wie sie typischerweise in multikristallinem Si vorliegen, unter gängigen Prozessierungsbedingungen möglich ist. Die Breite der ausscheidungsfreien Zone $(200 \mu \mathrm{m})$ liegt dabei im Bereich der Dicke gegenwärtiger multikristalliner Si-Scheiben, die zu Solarzellen weiterverarbeitet werden.

Die Breite der ausscheidungsfreien Zone unterhalb der mit einer Metall-Si-Phase bedeckten Oberfläche hängt vom Diffusionskoeffizienten der metallischen Verunreinigung (hier: $\mathrm{Cu}$ ) und der Abkühlrate (hier: $\dot{T}=-6 \mathrm{~K} / \mathrm{s}$ ) ab. Daraus ergibt sich die Perspektive, durch Kombination von Experimenten, bei denen die Abkühlrate variiert wird, mit numerischen Simulationen des Konzentrationsprofils der ausgeschiedenen metallischen Verunreinigung, die die Keimbildung berücksichtigen, die kritische Übersättigung $s_{\mu D}$ für die Keimbildung an Mikrodefekten zu bestimmen. Bei Anwesenheit der binären Gleichgewichtsphase auf der Oberfläche ist die Randbedingung für die Diffusion wohldefiniert im Gegensatz zur freien Oberfläche.

Am Anfang könnten Simulationen der reinen Ausdiffusion der metallischen Verunreinigung in eine Metallsilizidphase an der Oberfläche ohne Ausscheidungsbildung während der Abkühlung stehen. Die simulierten Werte der Verunreinigungskonzentration bei einem Abstand von der Oberfläche, der der Breite der beobachteten ausscheidungsfreien Zone entspricht, können eine erste Abschätzung für den Wert von $s_{\mu D}$ liefern. 


\subsection{Koausscheidung zweier metallischer Verunreinigungen}

\subsubsection{Zusammenfassung}

Die Erforschung der Eigenschaften von Metallsilizidausscheidungen in multikristallinem Silizium (mc-Si) für Solarzellen, das mit einer Vielzahl von metallischen Verunreinigungen zugleich kontaminiert ist [5, 7], ist ein Gegenstand aktueller Forschung [28, 29, 70, 72]. In dieser Arbeit wurde das Ausscheidungsverhalten im Fall jeweils zweier Spezies metallischer Fremdatome in Silizium untersucht. Methoden der Transmissionselektronenmikroskopie (TEM) in Verbindung mit energiedispersiver Röntgenanalytik (EDX) ermöglichten dabei einen Zugang zu Eigenschaften wie Größe, Form, Verteilung, Zusammensetzung und Struktur der Ausscheidungen. Für Metallsilizidausscheidungen in Si in binären Systemen ist bekannt, dass diese Eigenschaften von der Volumenfehlpassung $\delta_{V}$ der ausgeschiedenen Metallsilizidphase abhängen [26, 27]. Aus der Fragestellung, inwiefern die Volumenfehlpassung auch bei der Koausscheidung zweier metallischer Verunreinigungen eine Rolle spielt, ergab sich für diese Arbeit das experimentelle Vorgehen.

Proben des Si-Bikristalls wurden jeweils mit Kupfer und Nickel bzw. mit Palladium und Nickel durch Kodiffusion gezielt verunreinigt. $\mathrm{Cu}$ und $\mathrm{Ni}$ besitzen technologische Relevanz, da sie nach $\mathrm{Fe}$ zu den häufigsten Verunreinigungen in multikristallinem $\mathrm{Si}$ gehören. Durch die anschließende Abkühlphase wurden die Ausscheidungen präpariert, wobei die strukturellen Defekte und Oxidausscheidungen des Bikristall wie bei $\mathrm{Cu}$ als einziger Verunreinigung als heterogene Keimbildungsplätze dienten. Diffusionstemperatur $T$ und Abkühlrate $\dot{T}$ wurden so gewählt, dass sie gängigen Prozessbedingungen bei der Herstellung von Solarzellen entsprachen, $T=900^{\circ} \mathrm{C}$ bzw. $T=1050^{\circ} \mathrm{C}$ und $\dot{T}=-6 \mathrm{~K} / \mathrm{s}$. Die Metallsilizidausscheidungen, die sich im Verlauf der Abkühlung gebildet haben, zeigen dieselbe inhomogene Verteilung für den Fall von Kupfer als alleiniger metallischer Verunreinigung.

Aus den ternären Phasendiagrammen der Systeme Cu-Ni-Si [34] und Ni-Pd-Si [69] geht hervor, dass Si in der siliziumreichen Ecke mit den gleichen Phasen im Gleichgewicht steht, wie auf den siliziumreichen Seiten der entsprechenden binären Metall-Si-Systeme. Vom Standpunkt der Thermodynamik ist zunächst zu erwarten, dass bei der Koausscheidung diese Gleichgewichtsphasen gebildet werden. Sie zeichnen sich im Fall von $\mathrm{Cu}$ und Pd durch hohe und bei Ni durch geringe Volumenfehlpassung zu Si aus, $\mathrm{Cu}_{3} \mathrm{Si}$ : $\delta_{V}=150 \%, \mathrm{Pd}_{2} \mathrm{Si}: \delta_{V}=110 \%$ und $\mathrm{NiSi}_{2}: \delta_{V}=2 \%$ bei $T=1050^{\circ} \mathrm{C}$, so dass bei der Koausscheidung von $\mathrm{Cu}$ und $\mathrm{Ni}$ bzw. Pd und Ni unterschiedlich hohe Volumenfehlpassungen gleichzeitg wirksam sind. Ein Parameter ist das Konzentrationsverhältnis der metallischen Verunreinigungen. Experimentell wurden kupferreiche Bedingungen über 
die Löslichkeiten der metallischen Fremdatome $(\mathrm{Cu}$ und $\mathrm{Ni}$ ) in $\mathrm{Si}$ nach einer für die Einstellung des Gleichgewichts hinreichend langen Diffusionsdauer, vgl. Abschnitt 4.2.1, und nickelreiche Bedingungen durch ein Konzentrationsprofil, vgl. Abschnitt 4.2.2, eingestellt. Zur Realisierung nickelreicher Bedingungen durch Einstellung der Löslichkeiten diente Pd als zweite Verunreinigung, vgl. Abschnitt 4.3 Es besitzt hinsichtlich der Volumenfehlpassung ähnliche Eigenschaften wie $\mathrm{Cu}$, seine Löslichkeit in Si jedoch liegt im Gegensatz zu der von $\mathrm{Cu}$ unterhalb der von Ni.

\subsubsection{Kupferreiche Bedingungen}

Nach Kodiffusion von $\mathrm{Cu}$ und $\mathrm{Ni}$ bei $T=1050^{\circ} \mathrm{C}$ unter kupferreichen Bedingungen (Abschnitt 4.2.1) lassen sich von Versetzungen berandete Kolonien kleinerer Teilchen parallel zu $\{110\}$-Ebenen finden. Die Teilchen sind vorwiegend Ausscheidungen einer Phase, die sich aufgrund struktureller Merkmale als $\mathrm{Cu}_{3} \mathrm{Si}$ interpretieren lässt. Zusätzlich enthalten die Ausscheidungen einen kleinen aber signifikanten Anteil Ni, der einem partiellen Molenbruch von $3.2 \pm 0.2 \%$ entspricht. Der partielle Molenbruch wurde in G1. 4.2 (Abschnitt 4.2.1.3) als Konzentration einer metallischen Verunreinigung in einer Ausscheidung bezogen auf die Konzentration aller metallischen Fremdatome insgesamt definiert. Die Phase lässt sich daher als Lösung von $\mathrm{Ni}$ in $\mathrm{Cu}_{3} \mathrm{Si}$ beschreiben und wird in dieser Arbeit als $\mathrm{Cu}_{3} \mathrm{Si}$ :Ni bezeichnet. Die Ausscheidungen dieser Phase sind nahezu kugelförmig, wobei der Durchmesser im Mittel $20 \mathrm{~nm}$ beträgt. Der durchschnittliche Abstand zweier Teilchen liegt bei $74 \pm 9 \mathrm{~nm}$.

Einige der Teilchen in einer Kolonie haben eine polyedrische Form mit $\{111\}$-Oberflächen und enthalten wesentlich mehr Ni als $\mathrm{Cu}$. Der gelöste Anteil $\mathrm{Cu}$ entspricht einem partiellen Molenbruch von 15-23\%. Sie haben eine kubisch-flächenzentrierte Kristallstruktur mit vergleichbarer Gitterkonstante wie Si und zeigen zum Teil eine Zwillingsorientierung. Bei diesen Teilchen handelt es sich um Ausscheidungen einer Phase, sie sich als Lösung von $\mathrm{Cu}$ in $\mathrm{NiSi}_{2}$ beschreiben lässt und in dieser Arbeit als $\mathrm{NiSi}_{2}: \mathrm{Cu}$ bezeichnet wird. In ihrer Größe entsprechen sie ungefähr den $\mathrm{Cu}_{3} \mathrm{Si}$ :Ni-Teilchen und unterscheiden sich damit deutlich von $\mathrm{NiSi}_{2}$-Ausscheidungen, die sich bei vergleichbarer thermischer Behandlung unter nickelreichen Bedingungen (diese Arbeit) oder in mit $\mathrm{Ni}$ alleine verunreinigtem Si ([25]) gebildet haben und typischerweise $\geq 1 \mu \mathrm{m}$ sind. Der Mengenanteil der $\mathrm{NiSi}_{2}$ :Cu-Ausscheidungen an den Teilchen insgesamt beträgt etwa $8 \%$. Sie liegen dabei in der Regel nicht als isolierte Teilchen vor, sondern bilden mit $\mathrm{Cu}_{3} \mathrm{Si}: \mathrm{Ni}$ Ausscheidungen zusammenhängende Teilchen.

\subsubsection{Nickelreiche Bedingungen}

Nach Kodiffusion von $\mathrm{Pd}$ und Ni bei $T=1050^{\circ} \mathrm{C}$ unter nickelreichen Bedingungen (Abschnitt 4.3) haben sich nur wenige große Teilchen direkt an der Korngrenze gebildet. Wie die kleineren $\mathrm{NiSi}_{2}: \mathrm{Cu}$-Ausscheidungen nach Kodiffusion von $\mathrm{Cu}$ und $\mathrm{Ni}$ unter kupfer- 
reichen Bedingungen besitzen sie eine polyedrische Form mit $\{111\}$-Oberflächen, eine kubisch-flächenzentrierte Struktur mit Si vergleichbarer Gitterkonstante und liegen zum Teil in Zwillingsorientierung zur Si-Matrix vor, sind dabei aber mit typischen Abmessungen von ungefähr $1 \mu \mathrm{m}$ deutlich größer. Es handelt sich um Ausscheidungen einer Phase, die in ihrer Struktur und Zusammensetzung $\mathrm{NiSi}_{2}$ entspricht, wobei ein kleiner Anteil Pd von ca. $1.4 \pm 0.2$ At.-\% enthalten ist. Die Phase lässt sich daher als Lösung von $\mathrm{Pd}$ in $\mathrm{NiSi}_{2}$ deuten und wird hier als $\mathrm{NiSi}_{2}: \mathrm{Pd}$ bezeichnet. An den Oberflächen der großen $\mathrm{NiSi}_{2}: \mathrm{Pd}$-Ausscheidungen befinden sich außerdem kleinere palladiumreiche Teilchen, die ihrerseits $\mathrm{Ni}$ enthalten. In einem Fall nahe am Probenrand konnte für eines dieser Teilchen eine Zusammensetzung nachgewiesen werden, die ungefähr der von $\mathrm{Pd}_{2} \mathrm{Si}$ entspricht.

Nach Kodiffusion von $\mathrm{Cu}$ und Ni bei $T=900^{\circ} \mathrm{C}$ (Abschnitt 4.2.2) mit unvollständiger Gleichgewichtseinstellung lag in der Probe in einer Richtung ein Konzentrationsprofil von $\mathrm{Cu}$ bei fester Ni-Konzentration vor, so dass in einem Teil der Probe vor dem Beginn der Abkühlphase mit Bildung der Ausscheidungen nickelreiche Bedingungen vorlagen. Ausscheidungen aus diesem Teil der Probe haben die Form von Plättchen, die parallel zu $\{111\}$-Ebenen angeordnet sind. Diese Teilchen bestehen aus der $\mathrm{NiSi}_{2}$ :Cu-Phase, wobei der partielle Molenbruch von Cu hier $11 \pm 1 \%$ beträgt. Der Plättchendurchmesser liegt bei ungefähr $1 \mu \mathrm{m}$, so dass es sich wie bei den Ausscheidungen nach Kodiffusion von Pd und Ni um Teilchen mit einer für $\mathrm{NiSi}_{2}$ typischen Form und Größe handelt. Ebenfalls analog zur Kodiffusion von $\mathrm{Pd}$ und $\mathrm{Ni}$ sind auch hier die großen $\mathrm{NiSi}_{2}: \mathrm{Cu}$-Ausscheidungen zum Teil von kleineren kupferreichen Teilchen dekoriert, in denen wiederum ein kleiner Anteil $\mathrm{Ni}$ enthalten ist.

\subsubsection{Diskussion}

\subsubsection{Einfluss des Konzentrationsverhältnisses}

Nach der Koausscheidung von $\mathrm{Cu}$ und $\mathrm{Ni}$ unter kupferreichen Bedingungen zeigen die Proben eine Mikrostruktur, die vergleichbar ist mit derjenigen von Silizium nach alleiniger Ausscheidung von $\mathrm{Cu}$. Nach der Koausscheidung von $\mathrm{Pd}$ und $\mathrm{Ni}$ oder von $\mathrm{Cu}$ und Ni unter nickelreichen Bedingungen hingegen ähnelt die Mikrostruktur der Proben der von Silizium nach der Ausscheidung von Ni als einziger metallischer Verunreinigung. $\mathrm{Zu}$ einem vergleichbaren Ergebnis unter nickelreichen Bedingungen gelangt auch Stolze, die durch sequentielle Diffusion zuerst von $\mathrm{Ni}$ bei $1050^{\circ} \mathrm{C}$ und anschließend von $\mathrm{Cu}$ bei $750^{\circ} \mathrm{C}$ Ausscheidungen der $\mathrm{NiSi}_{2}$-Phase während der Reifung gezielt mit $\mathrm{Cu}$ verunreinigte [77], wobei unter Annahme der Daten für binäre Systeme das Verhältnis der Löslichkeiten von $\mathrm{Cu}$ und $\mathrm{Ni}$ in Si ungefähr 1:10 beträgt.

Neue mikrostrukturelle Phänomene sind im ternären Charakter der Koausscheidung begründet. Es kommt unabhängig vom System und vom Konzentrationsverhältnis der 
metallischen Verunreinigungen zur Bildung zusammenhängender Ausscheidungen unterschiedlicher Phasen und zur Lösung der jeweils anderen metallischen Komponente.

Heuer et al. ([28, 70]) und Buonassisi et al. ([29]) stellten auf zwei verschiedene Weisen Ausscheidungen in gleichzeitig bei $1200^{\circ} \mathrm{C}$ mit $\mathrm{Cu}$, Ni und Fe kontaminiertem multi- und einkristallinem Si her, nämlich

(i) durch sehr langsame Abkühlung nach der Eindiffusion mit einer Rate zwischen $\dot{T}=-0.01 \mathrm{~K} / \mathrm{s}$ und $\dot{T}=-0.5 \mathrm{~K} / \mathrm{s}$,

(ii) bei Beendigung der Eindiffusion durch Abschrecken durch eine nachfolgende Auslagerungsglühung bei $655^{\circ} \mathrm{C}$.

Sie untersuchen die Ausscheidungen mit Röntgenfluoreszenzmikroskopie und Röntgenabsorptionsspektroskopie und finden ebenfalls unterschiedliche Verhältnisse bezüglich Form, Größe und Verteilung der Ausscheidungen vor. Sie beschreiben die nach Auslagerung bei $655^{\circ} \mathrm{C}$ vorliegenden Ausscheidungen als Teilchen geringer Größe ("nanoprecipitates"), die in Form von Kolonien angeordnet sind. Nach sehr langsamer Abkühlung dagegen finden sie einzelne mikrometergroße Teilchen einer quaternären Phase mit einer Struktur und Zusammensetzung vergleichbar der von $\mathrm{NiSi}_{2}$ und zusätzlich enthaltenem $\mathrm{Cu}$ und Fe. Diese Ergebnisse entsprechen den Befunden der vorliegenden Arbeit für den Fall kupfer- bzw. nickelreicher Bedingungen.

Nach Ansicht von Buonassisi et al. ist die Bildung kleiner zu Kolonien angeordneter Ausscheidungen bzw. einzelner großer Ausscheidungen aus $\mathrm{NiSi}_{2}$ mit $\mathrm{Cu}$ und $\mathrm{Fe}$ als gelösten Verunreinigungen ein Effekt der unterschiedlichen thermischen Behandlung [29]. Sie diskutieren die Eigenschaften der Ausscheidungen nicht unter dem Gesichtspunkt der Konzentrationsverhältnisse der einzelnen metallischen Verunreinigungen, da die Autoren annehmen, dass diese über die Wahl der Diffusionstemperatur durch die jeweiligen binären Löslichkeiten festliegen. Diese Annahme muss jedoch aus mindestens zwei Gründen in Frage gestellt werden:

- Es ist zu erwarten, dass sich die Löslichkeiten metallischer Verunreinigungen im Fall ternärer (diese Arbeit) oder noch höherkomponentiger Systeme ([28, 29, 70]) von den Löslichkeiten in binären Systemen unterscheiden. Eine neuere Untersuchung von Abdelbarey et al. zur Umverteilung von Fe aus Si in die flüssige Phase aus $\mathrm{Al}$ und $\mathrm{Si}$ ("Aluminiumgettern") beispielsweise lässt vermuten, dass im ternären System Al-Fe-Si die Löslichkeit von Fe in Si um bis zu zwei Größenordnungen höher ist als im binären System Fe-Si [84]. Sowohl bei Heuer et al. und Buonassisi et al.[28, [29, 70], bei Stolze [77] als auch in dieser Arbeit wurden jedoch in Ermangelung von Daten zur Löslichkeit in ternären Systemen die Löslichkeitsparameter der binären Systeme als Grundlage verwendet.

- Metallsilizidphasen an der Oberfläche der Probe, die bei [28, 29, 70] und in dieser Arbeit als Quellen zur gezielten Verunreinigung dienen, wirken während eines Abkühlvorgangs als effektive Senken, wie am Beispiel der ausscheidungsfreien Zonen 
unterhalb der mit einer Cu-Si-Phase bedeckten Oberfläche in Abschnitt 5.1 diskutiert wurde. Daher können die Konzentrationen der im Silizium verbleibenden metallischen Verunreinigungen sich von den bei Eindiffusionstemperatur vorliegenden unterscheiden.

Auch in der vorliegenden Arbeit zeigen die Konzentrationsverhältnisse der metallischen Verunreinigungen Abweichungen von den nach binären Löslichkeitsparametern erwarteten Werten. So beträgt das Löslichkeitsverhältnis von $\mathrm{Cu}$ und $\mathrm{Ni}$ bei $1050^{\circ} \mathrm{C}$ nach Daten von Weber,[39] vgl. Tab. 4.1, etwa 2:1. Das Verhältnis von Cu und Ni in Ausscheidungen unter kupferreichen Bedingungen, vgl. Abschnitt 4.2.1.3, lässt sich dagegen ungefähr mit 8:1 abschätzen, wobei der durchschnittliche Wert des partiellen Molenbruchs von $\mathrm{Cu}$ in $\mathrm{Cu}_{3} \mathrm{Si}$ :Ni-Ausscheidungen $X_{C u}^{(p)}=96.8 \%$ und der Mengenanteil von 92\% der $\mathrm{Cu}_{3} \mathrm{Si}$ :Ni-Ausscheidungen an den Teilchen in einer Kolonie insgesamt eingehen. Der Einfluss des Konzentrationsverhältnisses zeigt sich besonders deutlich am Beispiel der Probe, in der nach Kodiffusion von $\mathrm{Cu}$ und Ni entlang der Korngrenze ein Konzentrationsprofil von $\mathrm{Cu}$ bei fester Ni-Konzentration eingestellt wurde, vgl. Abschnitt 4.2.2. An der Verteilung und Form der Ätzgrübchen lässt sich erkennen, dass hier bei gleicher thermischer Behandlung unter kupfer- und nickelreichen Bedingungen die Ausscheidungen unterschiedliche Eigenschaften besitzen.

\subsubsection{Gitterplatz von $\mathrm{Cu}$ und Volumenfehlpassung}

Die Beobachtung von $\mathrm{Cu}$ oder Ni als gelöste Komponente innerhalb von Metallsilizidausscheidungen der jeweils anderen metallischen Verunreinigung in Si wirft die Frage nach dem Gitterplatz der zusätzlich gelösten $\mathrm{Cu}$ - oder Ni-Atome auf. Im Fall der $\mathrm{NiSi}_{2}$ :CuAusscheidungen ergeben sich Besonderheiten hinsichtlich der Größe der Teilchen und der Konzentration des $\mathrm{Cu}$ beim Vergleich mit binären Metallsilizidausscheidungen und den Zusammensetzungen ternärer Legierungen, die in den nächsten beiden Abschnitten mit Bezug zum Gitterplatz der $\mathrm{Cu}$-Atome in der $\mathrm{NiSi}_{2}: \mathrm{Cu}-\mathrm{Phase}$ diskutiert werden.

Heuer et al., die Ausscheidungen einer Phase mit einer Zusammensetzung ähnlich der von $\mathrm{NiSi}_{2}$ und $\mathrm{Cu}$ und $\mathrm{Fe}$ als gelösten metallischen Verunreinigungen mittels Röntgenabsorptionsspektroskopie untersucht haben [28], ziehen aus dem Vergleich ihrer experimentellen mit simulierten Spektren den Schluss, dass Cu-Atome in diesem quaternären System Si-Plätze und Fe-Atome Ni-Plätze der $\mathrm{NiSi}_{2}$-Struktur besetzen. Wenn im ternären System $\mathrm{Cu}-\mathrm{Ni}$-Si Cu-Atome als gelöste metallische Verunreinigung in $\mathrm{NiSi}_{2}: \mathrm{Cu}-$ Ausscheidungen ebenfalls Si-Plätze besetzen, ergibt sich als Folge ein Beitrag zur Volumenfehlpassung der $\mathrm{NiSi}_{2}$ :Cu-Phase äquivalent der Erzeugung eines interstitiellen SiAtoms pro $\mathrm{Cu}$-Atom. Die Anzahl dieser interstiellen Si-Atome bezogen auf die Gesamtzahl der in einer $\mathrm{NiSi}_{2}$ :Cu-Ausscheidung enthaltenen Metallatome ist dabei wesentlich geringer als bei $\mathrm{Cu}_{3} \mathrm{Si}$ aber deutlich größer als bei $\mathrm{NiSi}_{2}$. Die Volumenfehlpassung einer 
Ausscheidung der $\mathrm{NiSi}_{2}$ :Cu-Phase hängt daher auch vom partiellen Molenbruch der gelösten Verunreinigung ab.

Bei der Koausscheidung von $\mathrm{Cu}$ und $\mathrm{Ni}$ in Si koexistieren Ausscheidungen zweier binärer Metallsilizidphasen, die beide die jeweils andere metallische Komponente enthalten. Es lässt sich eine mittlere Volumenfehlpassung $\delta_{V}^{a v}$ formulieren, die die unterschiedlichen Volumenfehlpassungen der einzelnen Phasen berücksichtigt, wie in Anhang C gezeigt wird. Unter der Voraussetzung, dass die Zusammensetzung der $\mathrm{Cu}_{3} \mathrm{Si}$ :Ni- und der $\mathrm{NiSi}_{2}$ :Cu-Ausscheidungen festliegt, kann $\delta_{V}^{a v}$ als Funktion des Konzentrationsverhältnisses der beiden Verunreinigungen ausgedrückt werden, vgl. Gl. (C.5). Unter der Annahme eines bestimmten Gitterplatzes der jeweils gelösten Verunreinigung ist die die Zusammensetzung der Ausscheidungen durch den partiellen Molenbruch von Cu bestimmt. Das Konzentrationsverhältnis $Q_{C u, N i}$ (Gl. 4.1 in Abschnitt 4.2) bezieht sich auf die insgesamt in Ausscheidungen beider Phasen vorliegenden Mengen von $\mathrm{Cu}$ - und NiAtomen. Abb. 5.1stellt den Verlauf der mittleren Volumenfehlpassung gerechnet für drei verschiedene Fälle bei Raumtemperatur dar:

- bei ternärer Löslichkeit von $\mathrm{Cu}$ in $\mathrm{NiSi}_{2}$ für die Besetzung von $\mathrm{Si}-\mathrm{Plätzen} \mathrm{des} \mathrm{NiSi}_{2}$ durch Cu-Atome wie bei Heuer et al. [28]

- alternativ für die Besetzung von Ni-Plätzen des $\mathrm{NiSi}_{2}$ durch $\mathrm{Cu}$-Atome

- ohne ternäre Löslichkeit von $\mathrm{Cu}$ in $\mathrm{NiSi}_{2}$ und $\mathrm{Ni}$ in $\mathrm{Cu}_{3} \mathrm{Si}$

Für den partiellen Molenbruch von $\mathrm{Cu}$ fanden die in dieser Arbeit gemessenen Werte von $X_{C u}^{(P)}=15 \%$ für $\mathrm{NiSi}_{2}$ :Cu-Ausscheidungen und $X_{\mathrm{Cu}}^{(P)}=96.8 \%$ für $\mathrm{Cu}_{3} \mathrm{Si}: \mathrm{Ni}$ Ausscheidungen Verwendung. Bei Vorliegen von Ni als gelöster Verunreinigung in $\mathrm{Cu}_{3} \mathrm{Si}$ ist der Einfluss des Gitterplatzes auf die Volumenfehlpassung der $\mathrm{Cu}_{3} \mathrm{Si}$ :Ni-Phase aufgrund des geringen Ni-Anteils vernachlässigbar. Bei der Berechnung wird angenommenen, dass Ni sich auf einem Cu-Platz einbaut.

Ausscheidungen der $\mathrm{NiSi}_{2}: \mathrm{Cu}$ - und der $\mathrm{Cu}_{3} \mathrm{Si}: \mathrm{Ni}$-Phase koexistieren nur innerhalb eines bestimmten Zusammensetzungsbereiches, wie das ternäre Phasendiagramm des Systems Cu-Ni-Si zeigt, vgl. Abb. 2.4 in Abschnitt 2.2.1. Die Zusammensetzung der $\mathrm{NiSi}_{2}: \mathrm{Cu}-$ bzw. der $\mathrm{Cu}_{3} \mathrm{Si}$ :Ni-Phase, die vom Wert des partiellen Molenbruchs von $\mathrm{Cu}$ in der jeweiligen Phase abhängt, gibt eine untere (hier: $Q_{C u, N i}=0.18$ ) bzw. obere Grenze (hier: $Q_{C u, N i}=30.3$ ) für mögliche Konzentrationsverhältnisse von $\mathrm{Cu}$ und $\mathrm{Ni}$ vor.

Unter nickelreichen Bedingungen $\left(Q_{C u, N i}<1\right)$ liegt $\mathrm{Cu}$ vorwiegend als gelöste Verunreinigung in $\mathrm{NiSi}_{2}$ :Cu-Ausscheidungen vor. Abb. 5.1 zeigt, dass die mittlere Volumenfehlpassung $\delta_{V}^{a v}$ in diesem Fall durch den Gitterplatz der Cu-Atome in der $\mathrm{NiSi}_{2}$ :Cu-Phase bestimmt wird. Bei Besetzung von Si-Plätzen liegt der berechnete Wert von $\delta_{V}^{a v}$ deutlich 


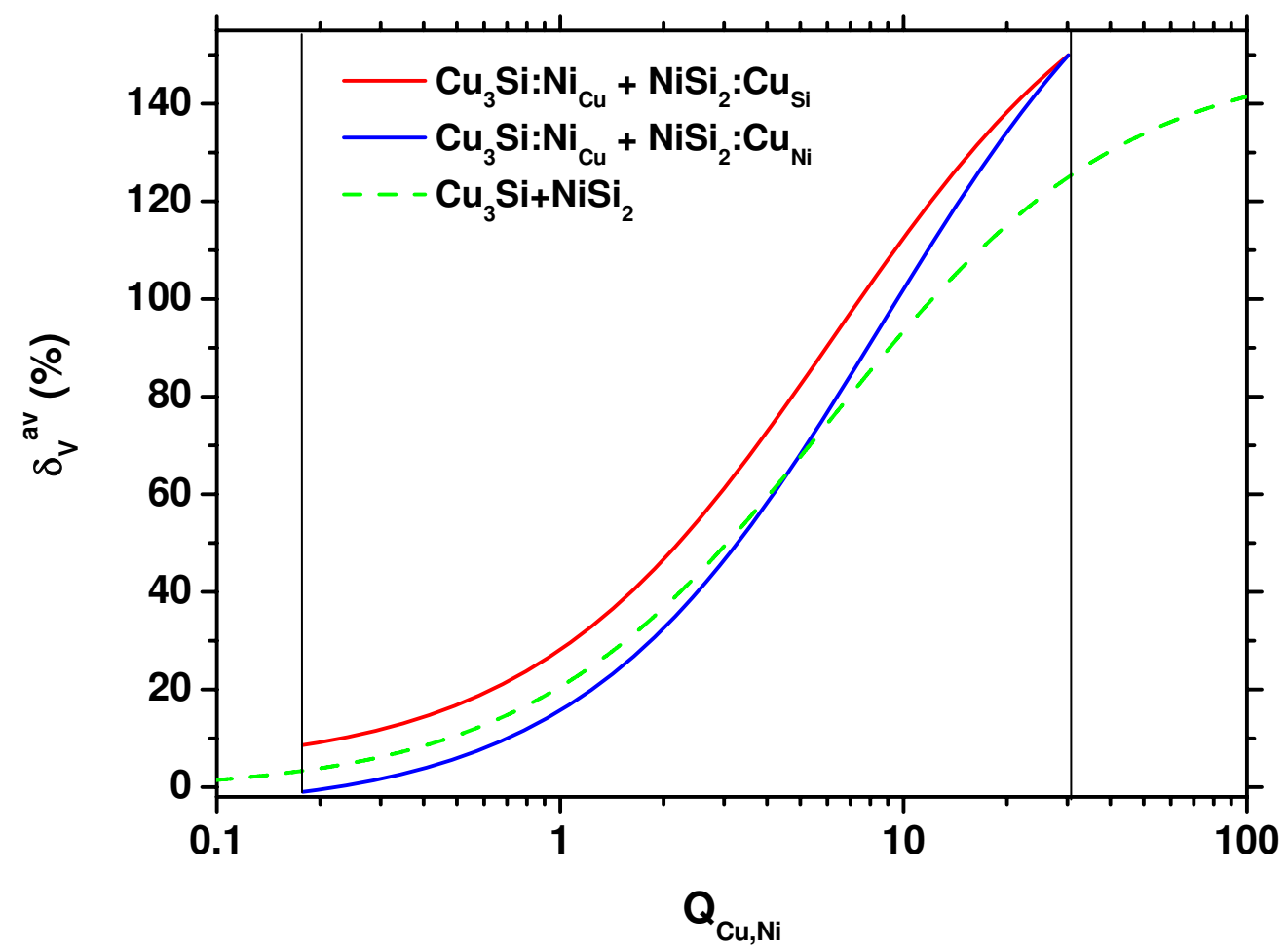

Abbildung 5.1. - Mittlere Volumenfehlpassung $\delta_{V}^{a v}$ bei Anwesenheit von Ausscheidungen der Phasen $\mathrm{Cu}_{3} \mathrm{Si}: \mathrm{Ni}$ und $\mathrm{NiSi}_{2}: \mathrm{Cu}$ bei Raumtemperatur als Funktion des Konzentrationsverhältnisses $Q_{C u, N i}$ des insgesamt ausgeschiedenen $\mathrm{Cu}$ und Ni für den Fall, dass $\mathrm{Cu}$-Atome in $\mathrm{NiSi}_{2}: \mathrm{Cu}$ Si-Plätze (rote Kurve) oder Ni-Plätze (blaue Kurve) besetzen, berechnet nach Gl. (C.5) aus Anhang C, Die vertikalen Linien markieren die Grenzen des Koexistenzbereiches der $\mathrm{Cu}_{3} \mathrm{Si}: \mathrm{Ni}$ - und $\mathrm{NiSi}_{2}: \mathrm{Cu}$-Phase im Gleichgewicht mit Si im ternären System. Die unterbrochene Kurve gibt $\delta_{V}^{a v}$ für die parallele Bildung binärer Metallsilizidausscheidungen ( $\mathrm{NiSi}_{2}$ und $\mathrm{Cu}_{3} \mathrm{Si}$ ) ohne ternäre Löslichkeit an.

über der Volumenfehlpassung der binären $\mathrm{NiSi}_{2}$-Phase Der geringste Wert von $\delta_{V}^{a v}$ ergibt sich bei der Besetzung von Ni-Plätzen durch Cu-Atome

Unter kupferreichen Bedingungen für Konzentrationsverhältnisse nahe an der oberen Grenze des Koexistenzbereiches liegt $\mathrm{Cu}$ vorwiegend in Form von $\mathrm{Cu}_{3} \mathrm{Si}$ :Ni-Ausscheidungen vor. Der Unterschied der mittleren Volumenfehlpassung $\delta_{V}^{a v}$, der aus der Besetzung unterschiedlicher Plätze des $\mathrm{NiSi}_{2}$ durch $\mathrm{Cu}$-Atome resultiert, verschwindet und der Wert von $\delta_{V}^{a v}$ erreicht den der Volumenfehlpassung der $\mathrm{Cu}_{3}$ Si-Phase (150\%, vgl. Abschnitt 2.1.2.3), da fast alle Ausscheidungen der $\mathrm{Cu}_{3} \mathrm{Si}$ :Ni-Phase angehören. 


\subsubsection{Vergleich von $\mathrm{NiSi}_{2}: \mathrm{Cu}-$ und $\mathrm{NiSi}_{2}$-Ausscheidungen}

Es ist bekannt, dass bei der Ausscheidung von $\mathrm{Cu}$ als alleiniger metallischer Verunreinigung in $\mathrm{Si}$ die hohe Volumenfehlpassung der $\mathrm{Cu}_{3}$ Si-Phase $\mathrm{zu}$ wiederholter Bildung und Wachstum kleiner Ausscheidungen an Versetzungen führt, vgl. Abschnitt 2.1.2.3, [42, 43, 55, 56]. Die Versetzung absorbiert die von den wachsenden Ausscheidungen emittierten interstitiellen Si-Atome und klettert dabei, so dass sich von Versetzungen berandete planare Kolonien kleiner Ausscheidungen ergeben. Durch den gleichen Mechanismus lässt sich auch die Entstehung der ausgedehnten Kolonien kleiner Ausscheidungen der $\mathrm{Cu}_{3} \mathrm{Si}$ :Ni-Phase erklären, die in dieser Arbeit nach Kodiffusion von $\mathrm{Cu}$ und Ni unter kupferreichen Bedingungen beobachtet werden, vgl. Abschnitt 4.2.1.

Unter der Annahme, dass $\mathrm{Cu}$-Atome in der $\mathrm{NiSi}_{2}$ :Cu-Phase auf Si-Plätzen vorliegen, werden beim Wachstum der $\mathrm{NiSi}_{2}$ :Cu-Ausscheidungen ebenfalls interstitielle Si-Atome erzeugt. Die Emission der interstitiellen Si-Atome entspricht dabei einer Volumenfehlpassung von ca. 10\%, wie Abb. 5.1 zeigt. Dieser Wert liegt deutlich über der Volumenfehlpassung der rein binären Phase $\mathrm{NiSi}_{2}$, die bei Raumtemperatur $-1.1 \%$ beträgt, vgl. Abschnitt 2.1.2.2. In der Gegenwart von Versetzungen wie im Fall der Ausscheidungskolonien unter kupferreichen Bedingungen, ist es daher möglich, dass auch die $\mathrm{NiSi}_{2}$ :CuAusscheidungen sich entlang kletternder Versetzungen bilden und in Kolonien integriert werden wie $\mathrm{Cu}_{3} \mathrm{Si}$ :Ni-Teilchen, anstelle einige wenige mikrometergroße Ausscheidungen zu bilden, wie es typischerweise bei der Ausscheidung von $\mathrm{Ni}$ als alleiniger metallischer Verunreinigung der Fall ist, vgl. Abschnitt 2.1.2.2. In der Tat lassen sich nach Kodiffusion von $\mathrm{Cu}$ und $\mathrm{Ni}$ unter kupferreichen Bedingungen $\mathrm{NiSi}_{2}$ : $\mathrm{Cu}$-Ausscheidungen finden, die von vergleichbarer Größe und in gleicher Weise innerhalb einer Kolonie angeordnet sind wie $\mathrm{Cu}_{3} \mathrm{Si}$ :Ni-Ausscheidungen, vgl. Abb. 4.15 in Abschnitt 4.2.1.3. Die $\mathrm{NiSi}_{2}: \mathrm{Cu}-$ Ausscheidungen zeigen dabei die gleichen wohlbekannten strukturellen Merkmale wie $\mathrm{NiSi}_{2}$-Plättchen nach Ausscheidung von $\mathrm{Ni}$ als alleiniger metallischer Verunreinigung, sind jedoch mit Abmessungen von 30-60 nm wesentlich kleiner.

Unter nickel- wie unter kupferreichen Bedingungen hat die Volumenfehlpassung der $\mathrm{NiSi}_{2}$ :Cu-Ausscheidungen den gleichen Wert von etwa 10\%. Sie wird jedoch auf unterschiedliche Weise kompensiert. Während die Ergebnisse unter kupferreichen Bedingungen auf die Bildung kleiner $\mathrm{NiSi}_{2}: \mathrm{Cu}$-Teilchen an Versetzungen in Kolonien hindeuten, kommt es unter nickelreichen Bedingungen ohne Kolonien zu plastische Verformung, was sich beispielsweise an den Versetzungen in der Nähe der $\mathrm{NiSi}_{2}: \mathrm{Pd}$-Ausscheidungen nach Kodiffusion von Pd und Ni zeigt, vgl. Abschnitt 4.3. 


\subsubsection{Konzentration von $\mathrm{Cu}$ in $\mathrm{NiSi}_{2}: \mathrm{Cu}$-Ausscheidungen}

Nach der Koausscheidung von $\mathrm{Cu}$ und $\mathrm{Ni}$ bestehen die Ausscheidungen sowohl unter kupfer- wie unter nickelreichen Bedingungen aus denselben ternären Phasen, die sich nach dem Klassifikationsschema von Setton als Lösungen von $\mathrm{Cu}$ und Ni in Siliziden der jeweils anderen metallischen Verunreinigung beschreiben lassen [58] und isostrukturell zu den jeweiligen binären Siliziden sind. Der dem ternären Phasendiagramm von Sokolovskaya et al. [34] entnommene Wert für die Löslichkeit von $\mathrm{Cu}$ in $\mathrm{NiSi}_{2}$ bei $500^{\circ} \mathrm{C}$ in einer Legierung aus $\mathrm{Cu}, \mathrm{Ni}$ und $\mathrm{Si}$ entspricht einem partiellen Molenbruch $\mathrm{X}_{\mathrm{Cu}}^{(p)}$ von $3 \%$. Die Werte von $X_{\mathrm{Cu}}^{(p)}$ im Fall der in Abschnitt 4.2 betrachteten $\mathrm{NiSi}_{2}$ :Cu-Ausscheidungen liegen im Bereich zwischen 11 und 23\%, vgl. Tab. 4.3 und Tab. 4.4. Der Anteil von $\mathrm{Cu}$ an der Zusammensetzung der von Heuer et al. und Buonassisi et al. untersuchten Ausscheidungen einer quaternären Phase aus $\mathrm{Fe}, \mathrm{Cu}, \mathrm{Ni}$ und Si normiert auf den Anteil metallischer Fremdatome insgesamt variiert zwischen 2 und 15\% [28, 29, 70].

Die Konzentration von $\mathrm{Cu}$ in Ausscheidungen der $\mathrm{NiSi}_{2}$ :Cu-Phase übertrifft so die Löslichkeit von $\mathrm{Cu}$ in $\mathrm{NiSi}_{2}$ für $500^{\circ} \mathrm{C}$ um bis zu eine Größenordnung. Diese Beobachtung ist nicht überraschend, wenn man beachtet, dass im vorliegenden Fall die Bildung der Phasen in Form von Ausscheidungen und während eines Abkühlvorgangs von $1050^{\circ} \mathrm{C}$ auf Raumtemperatur nach Kodiffusion von $\mathrm{Cu}$ und Ni stattgefunden hat:

- Isothermische Schnitte durch ein ternäres Phasendiagramm geben die Zusammensetzung der Gleichgewichtsphasen bei fester Temperatur an. Es ist offen, ob sich das System ins Gleichgewicht gebracht hat und wenn ja, bei welcher Temperatur. Die Einstellung der Löslichkeit durch $\mathrm{Cu}$ in Ausscheidungen der $\mathrm{NiSi}_{2}: \mathrm{Cu}-\mathrm{Phase}$ ist wahrscheinlicher, wenn diese mit Ausscheidungen der $\mathrm{Cu}_{3} \mathrm{Si}$ :Ni-Phase in direktem Kontakt stehen. Dies zeigt sich am Beispiel der Ausscheidungen P1, P2 in Abschnitt 4.2.1.3, in denen ein niedrigerer Anteil $\mathrm{Cu}$ enthalten ist, als in der isolierten Ausscheidung P3, vgl. Tab. 4.3, Die Differenz des Wertes des partiellen Molenbruchs $X_{C u}^{(P)}$ von Cu der Ausscheidungen P1 und P3 bzw. P2 und P3 entspricht einer Menge von $\mathrm{Cu}$-Atomen, die zur Bildung einer $\mathrm{Cu}_{3}$ Si-Ausscheidung mit einem Durchmesser von $25 \mathrm{~nm}$ im Fall von P1 und $20 \mathrm{~nm}$ im Fall von P2 ausreicht. Diese Werte weisen eine gute Übereinstimmung mit der Größe der tatsächlich an P1 und P2 angrenzenden $\mathrm{Cu}_{3} \mathrm{Si}$ :Ni-Ausscheidungen auf, vgl. Abb. 4.6 und Abb. 4.8.

- Phasendiagramme lassen Beiträge zur freien Enthalpie des Systems unberücksichtigt, die sich bei der Einbettung von Ausscheidungen in die Matrix ergeben, wie etwa die Grenzflächenenergie und Verzerrung infolge von Volumenfehlpassung. Wie in Abschnitt 5.2.2.2 diskutiert, hängt die Volumenfehlpassung der $\mathrm{NiSi}_{2}$ :Cu-Phase vom Gitterplatz des $\mathrm{Cu}$ ab. Unter der Annahme eines Si-Platzes wie bei Heuer et al. [28] entspricht die Volumenfehlpassung der $\mathrm{NiSi}_{2}$ :Cu-Phase der Erzeugung eines interstiellen Si-Atoms pro Cu-Atom. Aus ihr ergibt sich ein zusätzlicher positiver 
Beitrag zur freien Enthalpie des gesamten Systems. Durch ihn verringert sich nach Wagner et al. die treibende Kraft [85], ein $\mathrm{Cu}$-Atom in der $\mathrm{NiSi}_{2}: \mathrm{Cu}$ zu lösen. Im Vergleich $\mathrm{zu}$ einer Legierung ist daher eine geringere Löslichkeit von $\mathrm{Cu}$ in der $\mathrm{NiSi}_{2}: \mathrm{Cu}-\mathrm{Phase}$ zu erwarten, wenn diese in Form von Ausscheidungen vorliegt. Unter kupferreichen Bedingungen allerdings wird dieser Effekt durch die Anwesenheit der Versetzungsringe innerhalb der Kolonien abgeschwächt. Versetzungen stellen Senken für die von den Ausscheidungen erzeugten interstiellen Si-Atome dar, welche so ihre Gleichgewichtskonzentration einstellen können. Die Energie eines Versetzungsrings nimmt langsamer zu als die Anzahl der absorbierten interstiellen Si-Atome, so dass der zusätzliche positive Beitrag zur freien Enthalpie des System pro Cu-Atom mit zunehmender Größe des Versetzungsrings sinkt.

Die Annahme des Si-Platzes für $\mathrm{Cu}$-Atome in $\mathrm{NiSi}_{2}: \mathrm{Cu}$ ermöglicht eine konsistente Deutung der Beobachtung, dass sich unter kupferreichen Bedingungen relativ kleine und in Kolonien integrierte $\mathrm{NiSi}_{2}: \mathrm{Cu}$-Ausscheidungen bilden, sie kann jedoch nicht die hohe Konzentration von $\mathrm{Cu}$ in $\mathrm{NiSi}_{2}$ :Cu erklären. Eine andere Beobachtung spricht dafür, dass die hohe Konzentration von $\mathrm{Cu}$ in der Tat eine Folge der thermischen Behandlung ist:

Anders als im System Cu-Ni-Si stimmt im System Ni-Pd-Si die in $\mathrm{NiSi}_{2}$ :Pd-Ausscheidungen gemessene Pd-Konzentration von ungefähr 1.4 \pm 0.2 At.\%(Abschnitt 4.3.3) gut mit der der Löslichkeit von $\mathrm{Pd}$ in $\mathrm{NiSi}_{2}$ in Legierungen überein, für die Loomans et al. Werte zwischen 1.0 At. $\%$ bei $760^{\circ} \mathrm{C}$ und 1.4 At. $\%$ bei $880^{\circ} \mathrm{C}$ angeben [68]. Die von Loomans et al. verwendeten Temperaturen liegen dabei wesentlich näher an der in dieser Arbeit für die Kodiffusion benutzen Temperatur von $1050^{\circ} \mathrm{C}$ als im Fall der Cu-Ni-SiLegierungen bei Sokolovskaya et al. $\left(500^{\circ} \mathrm{C}\right)$.

\subsubsection{Ausblick: Gitterplatzbestimmung mit TEM}

Auch die Ergebnisse der Untersuchung der Zusammensetzung der Metallsilizidausscheidungen mit TEM in Verbindung mit energiedispersiver Röntgenanalytik (EDX) in dieser Arbeit enthalten Informationen über den Gitterplatz von Fremdatomen in den Ausscheidungen:

- Lässt sich die Zusammensetzung einer Ausscheidung hinreichend genau aus der Analyse eines EDX-Spektrums nach der Methode von Cliff und Lorimer [78] bestimmen, erlauben Abweichungen der Zusammensetzung von der exakten Stöchiometrie einer binären Phase einen Rückschluss auf den Gitterplatz einer dritten Komponente. Dies setzt voraus, dass die Größe der Teilchen die Dicke der TEM-Probe übertrifft, so dass der Elektronenstrahl zwar die Ausscheidung jedoch nicht zugleich die Matrix durchstrahlt, wie beispielsweise im Fall der $\mathrm{NiSi}_{2}$ : $\mathrm{Cu}-$ Ausscheidungen nach Kodiffusion von $\mathrm{Cu}$ und Ni unter nickelreichen Bedingungen, Abschnitt 4.2.2. 
- Messungen der Intensität der $\mathrm{Si}_{K \alpha}$-Line entlang eines Schnitts durch eine Ausscheidung zeigen Unterschiede in der Dichte der Si-Atome zwischen Ausscheidungen und Matrix an, beispielsweise im Fall der $\mathrm{Cu}_{3} \mathrm{Si}$-Ausscheidungen nach Kodiffusion von $\mathrm{Cu}$ und Ni unter kupferreichen Bedingungen, vgl. Abschnitt 4.2.1.3. Interpretiert man diese Unterschiede als Folge von Volumenfehlpassung, dann entspricht eine Verringerung der $\mathrm{Si}_{K \alpha}$-Intensität einer Emission interstitieller Si-Atome. Sie würde im Fall einer Ausscheidung der $\mathrm{NiSi}_{2}: \mathrm{Cu}$-Phase mit $\mathrm{Cu}$ als gelöster metallischer Verunreinigung einen Hinweis darauf geben, das Cu-Atome Si-Plätze einnehmen.

Bei beiden Vorgehensweisen ist zu beachten, dass die elektronenmikroskopischen Beugungsbedingungen während der EDX-Messung die Intensität der EDX-Signale beeinflussen. Die laterale Verteilung der Intensität des Elektronenstrahls innerhalb der Probe hängt durch einen bei Spence und Taftø als „Channeling“ bezeichneten Effekt von der Orientierung der Probe bezüglich des einfallenden Elektronenstrahls ab [86]. In der vorliegenden Arbeit wurden die Beugungsbedingungen nicht hinreichend kontrolliert, um den Effekt des Channeling bei der Auswertung der EDX-Spektren berücksichtigen zu können.

Durch Variation der Elektronendichte auf verschiedenen Untergittern mittels systematischer Änderung der Beugungsbedingungen besteht die Möglichkeit, Fremdatome in einem geordneten Kristall zu lokalisieren. Dieses Verfahren ist in der Literatur als ALCHEMI ("Atom Localization by Channeling Enhanced Microanalysis") bekannt.

\subsubsection{Ausblick: Koausscheidung in Si für die Photovoltaik}

Es ist ein Ziel der gegenwärtigen Siliziumforschung, die metallischen Verunreinigungen in kommerziell für Solarzellen genutztem Si im Rahmen der Prozessierung so umzuverteilen, dass sie sich in wenigen großen Ausscheidungen sammeln. Derartige Bestrebungen werden in der Literatur als Defektmanipulation (,defect engineering“) bezeichnet [22]. Die vorliegenden Arbeit zeigt, dass es bei Koausscheidung zweier metallischen Verunreinigung zur gegenseitigen Lösung der metallischen Komponenten in Ausscheidungen kommt: $\mathrm{Cu}$ löst sich in $\mathrm{NiSi}_{2}$ und $\mathrm{Ni}$ in $\mathrm{Cu}_{3} \mathrm{Si}$. Die Thermodynamik ternärer System bietet einen Weg, verschiedene metallische Fremdatome in denselben Ausscheidungen zu konzentrieren. Ortsaufgelöste Untersuchungen des Photostroms an gleichzeitig mit $\mathrm{Cu}$ und Ni verunreinigten Bikristallen zeigen dabei eine hohe Rekombinationsaktivität der Ausscheidungen, vergleichbar der von Ausscheidungen in Si mit $\mathrm{Cu}$ oder Ni als alleiniger metallischer Verunreinigung [82]. Vom Standpunkt der Solarzellenherstellung her ist eine weit gestreute Verteilung kleiner rekombinationsaktiver Metallsilizidpartikel, wie sie nach Kodiffusion von $\mathrm{Cu}$ und $\mathrm{Ni}$ unter kupferreichen Bedingungen in Form von Kolonien kleiner $\mathrm{Cu}_{3} \mathrm{Si}: \mathrm{Ni}$ - und $\mathrm{NiSi}_{2}: \mathrm{Cu}$-Ausscheidungen vorliegt, also eine ungünstige Situation.

Bisherige Überlegungen zur Defektmanipulation befassen sich mit dem Einfluss der Abkühlrate, um durch Ostwaldreifung metallische Fremdatome von kleinen zu großen 
Teilchen hin umzuverteilen. In dieser Arbeit wird gezeigt, dass sich innerhalb derselben Probe - abhängig von der lokalen Relation der Konzentrationen der Cu- und Ni-Atome - einerseits Kolonien kleiner Teilchen und andererseits große isolierte Ausscheidungen bei gleicher thermischer Behandlung bilden können, Abschnitt 4.2.2, Das Konzentrationsverhältnis, hier: das Vorliegen kupfer- oder nickelreicher Bedingungen, beeinflusst die Form und Verteilung der Ausscheidungen. Es besteht also prinzipiell die Möglichkeit durch gezielte Verunreinigung mit Ni bei höherer Temperatur (höhere Löslichkeit) während der Prozessierung nickelreiche Bedingungen herbeizuführen und so die Bildung großer isolierter Ausscheidungen zu begünstigen. 
Anhänge 

$\Gamma_{\text {Anhang }} \mathrm{A}$

\section{Herstellung von Si-Bikristallen}

Für die Diffusionsexperimente wurde bikristallines Silizium als Probenmaterial benutzt. Die Herstellung erfolgte am MPI für Mikrostrukturphysik in Halle in der Gruppe von M. Reiche mittels Wafer-Bonding. Allgemeine Grundlagen und die Anwendung dieser Methode auf verschiedene Halbleitermaterialien beschreibt Reiche in einer Arbeit aus dem Jahr 2006 [87]. Experimentelle Einzelheiten des Verfahrens, das zur Herstellung des in dieser Arbeit verwendeten Materials eingesetzt wurde, schildern Reiche et al. bereits in einer Arbeit aus dem Jahr 1997 [33]. Das Vorgehen wird im Folgenden in zusammengefasster Form dargestellt.

Das Ausgangsmaterial waren n-leitende, $650 \mu \mathrm{m}$ dicke und (001)-orientierte Scheiben aus einkristallinem nach dem Czochralski-Verfahren hergestelltem Silizium, wobei die Oberflächen der Scheiben gegenüber der (001)-Ebene eine geringe Verkippung aufweisen. Nach der Entfernung von Oxidschichten durch Ätzen mit Flusssäure hatten die Si-Scheiben hydrophobe und Wasserstoff-terminierte Oberflächen. Zwei Scheiben wurden um einen Winkel von $1.5^{\circ}$ um die (001)-Achse gegeneinander verdreht und mit den Oberflächen so zusammengelegt, dass die (001)-Ebenen der beiden Scheiben um $0.04^{\circ}$ gegeneinander verkippt sind. Durch die Wasserstoff-Terminierung entstand zunächst eine schwachen Bindung zwischen den Scheiben aufgrund von van-der-Waals-Kräften zwischen Si-H-Komplexen. Eine thermische Behandlung bei $1050^{\circ} \mathrm{C}$ von 4 Stunden Dauer in einer Umgebung aus Sauerstoff führte zur Entstehung kovalenter Bindungen zwischen den Si-Atomen an den Oberflächen der beiden Scheiben. An der Grenzfläche der Scheiben liegt ein Versetzungsnetzwerk vor, das aus einer regelmäßigen Anordnung von Schrauben- und Stufenversetzungen besteht, wobei der Abstand zwischen den Schraubenversetzungen 12nm und zwischen den Stufenversetzungen $372 \mathrm{~nm}$ beträgt. Diese Versetzungsnetzwerk entspricht einer Kleinwinkelkorngrenze.

Abb. A.1 zeigt schematisch die Anordnung der beiden Scheiben (A und B) zu ei- 


\title{
Of-1
}

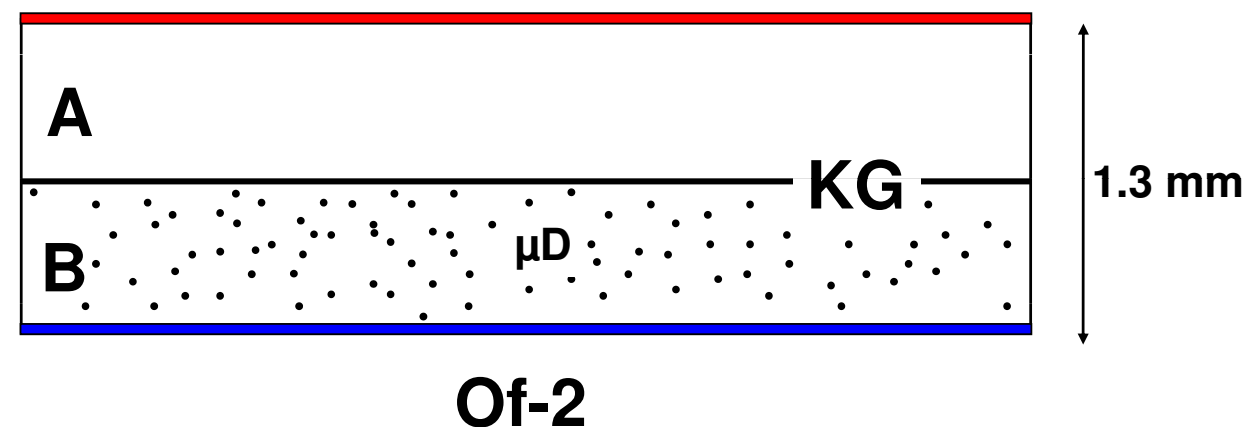

\begin{abstract}
Abbildung A.1. - Schematischer Querschnitt durch den in der vorliegenden Arbeit verwendeten Bikristall. An der Grenzfläche der beiden durch Waferbonding miteinander verbundenen Si-Scheiben A und B liegt eine Kleinwinkelkorngrenze (KG) vor. Eine der Scheiben (B) enthält eine hohe Dichte von Mikrodefekten $(\mu D)$. Bei ihnen handelt es um Oxidausscheidungen und kristallographische Defekte, die sich auf das Wachstum der Ausscheidungen zurückführen lassen. Für die Diffusionsexperimente werden auf den mit Of-1 und Of-2 bezeichneten Oberflächen Metallschichten als Quellen aufgebracht.
\end{abstract}

nem Bikristall. Auf den mit Of-1 und Of-2 bezeichneten Oberflächen wurden die bei den Diffusionsexperimenten dieser Arbeit als Quellen der metallischen Verunreinigung verwendeten Metallschichten aufgebracht. In der mit B bezeichneten Scheibe liegen Mikrodefekte $(\mu \mathrm{D})$ vor, die zum Teil Sauerstoff enthalten, wie die in Abb. A.2 dargestellte Untersuchung mit TEM und EDX zeigt. In der anderen Scheibe (A) konnten solche Defekte nicht gefunden werden.

Czochralski-Silizium (Cz-Si) enthält typischerweise Sauerstoff in Konzentration zwischen $10^{17}$ und $10^{18} \mathrm{~cm}^{-3}$ [88]. Es ist bekannt, dass in $\mathrm{Cz}-\mathrm{Si}$ durch geeignete thermische Behandlung Siliziumoxidausscheidungen gebildet werden können, deren Wachstum zu sekundären kristallographischen Defekten wie beispielsweise Versetzungsringen führen kann, die von den Oxidteilchen beim Wachstum ausgestoßen werden [88]. Die beiden miteinander verbundenen Scheiben bestehen aus Silizium, das nach dem CzochralskiVerfahren hergestellt wurde. Daher lassen sich die in Scheibe B des Bikristalls vorliegenden Mikrodefekte als Oxidausscheidungen und als Versetzungsringe identifizieren. Die Dichte der Mikrodefekte kann aufgrund des untersuchten Volumens der TEM-Probe mit etwa $10^{10} \mathrm{~cm}^{-3}$ abgeschätzt werden. Wie die Korngrenze zwischen Scheibe A und B stellen die Mikrodefekte Keimbildungsplätze für Metallsilizidausscheidungen dar. 


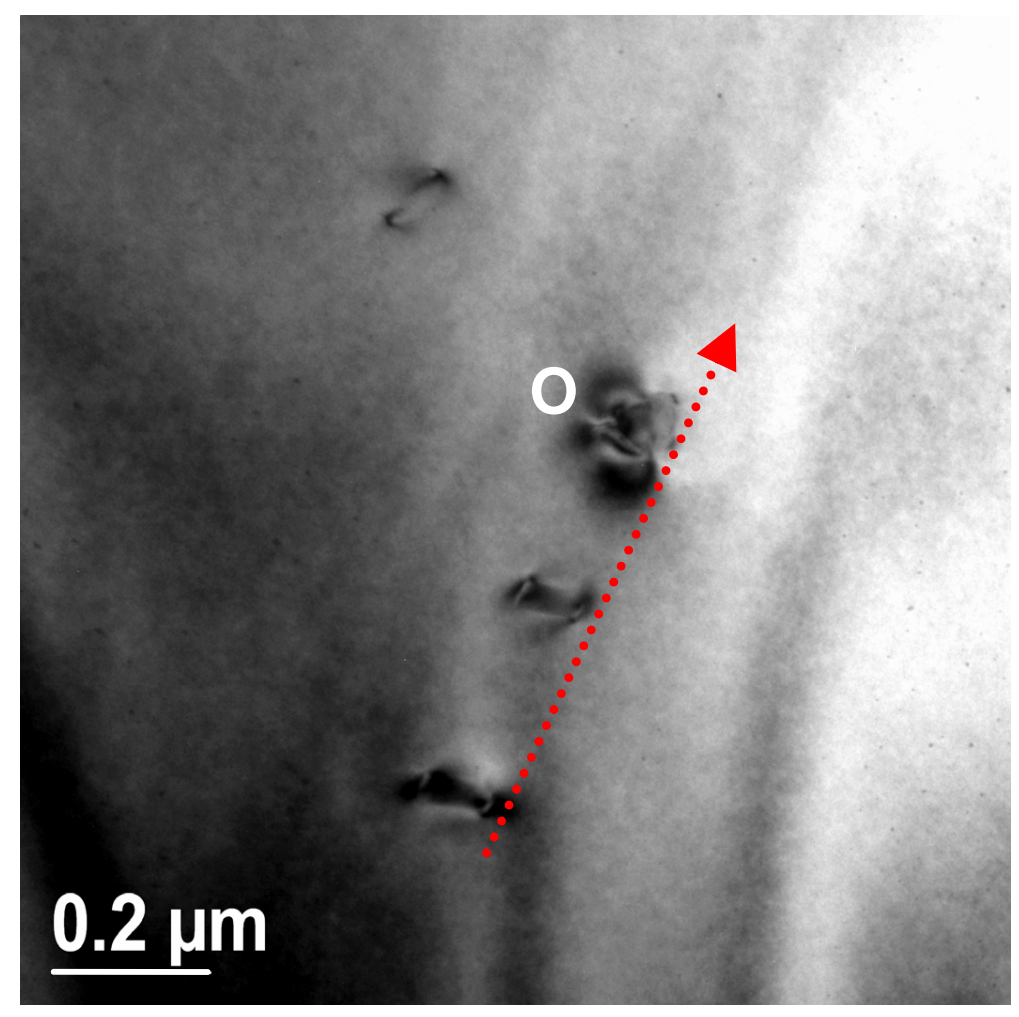

(a) TEM



(b) EDX

Abbildung A.2. - (a) TEM-Aufnahme von Mikrodefekten im Volumen der Scheibe B des in dieser Arbeit verwendeten Si-Bikristalls, vgl. Abb. A.1, (b) Die Untersuchung der Zusammensetzung der Probe mit EDX entlang einer Linie in der in (a) angegebenen Richtung zeigt, dass der mit $O$ beeichnete Mikrodefekt Sauerstoff enthält. 
Anhang 12

\section{Probenpräparation}

\section{B.1. Präparation der Diffusionsproben}

Aus dem Si-Bikristall wurden quaderförmige Stücke der Größe 10x10x1.3 mm³ abgebrochen, die als Diffusionsproben verwendet wurden. Die Ober- und Unterseite der Proben sind (001)-orientiert und die Seitenkanten liegen parallel zu $\langle 110\rangle$-Richtungen.

Die Probenoberflächen waren nach der Wafer-Bonding-Prozedur mit einem dicken Oxidfilm bedeckt, der nun durch verdünnte Flusssäure (HF(40\%): $\left.\mathrm{H}_{2} \mathrm{O}, 1: 10,10 \mathrm{~min}\right)$ entfernt wurde. Anschließend wurden die Proben im Ultraschallbad mit einer Sequenz organischer Lösungsmittel (Propanol, Aceton, Methanol) jeweils drei Minuten von groben Verunreinigungen gesäubert, bevor in einer Ätzlösung aus Flusssäure und Salpetersäure ( $\mathrm{HF}(40 \%): \mathrm{HNO}_{3}(65 \%)$ 1:10) für 10 Minuten je $50 \mu \mathrm{m}$ auf jeder Probenoberfläche abgetragen wurde [89]. Ein erneutes Bad in verdünnter Flusssäure $\left(\mathrm{HF}(40 \%): \mathrm{H}_{2} \mathrm{O}, 1: 10,10\right.$ min) zur Entfernung des Oxidfilmes, der nach der Ätzung im Flusssäure-SalpetersäureGemisch auf der Oberfläche zurückgeblieben war, schließst die Probenreinigung ab. Die verwendeten Lösungsmittel und Säuren waren Chemikalien der Fa. Merck in pro-analysiQualität bei Lösungsmitteln bzw. Suprapur-Qualität bei Säuren.

Als Diffusionsquellen wurden Metallschichten auf die Proben aufgedampft. Dazu diente die bereits von Riedel [90] für Ni-Diffusionsexperimente verwendete Aufdampfapparatur. Für die Kodiffusions-Experimente in dieser Arbeit wurden zwei Metalle (entweder $\mathrm{Cu}$ und $\mathrm{Ni}$ oder $\mathrm{Pd}$ und $\mathrm{Ni}$ ) nacheinander auf gegenüberliegende Probenoberflächen aufgedampft. Nach dem Bad in verdünnter Flusssäure wurden die Proben auf einen Teflonhalter montiert und möglichst schnell in die Aufdampfapparatur eingebaut. Der Teflonhalter war zuvor wie die Probe mit organischen Lösungsmitteln (Propanol, Aceton, Methanol) gereinigt und danach mit Königswasser ( $\mathrm{HCl}(30 \%): \mathrm{HNO}_{3}(65 \%), 3: 1,5$ min) geätzt worden um metallische Verunreinigungen zu entfernen. Nach Evakuierung der 
Aufdampfapparatur bis zu einem Druck unterhalb von $1.0 \cdot 10^{-6}$ mbar wurden $1-3 \mathrm{~mm}$ lange Drahtstücke des jeweiligen Metalls in einem Verdampferschiffchen aus Wolfram thermisch verdampft. Dabei stieg der Druck typischerweise um bis zu eine Größenordung an $\left(1.0 \cdot 10^{-5}\right)$. Für Kodiffusions-Experimente wurde die Anlage nach dem ersten Aufdampfen belüftet, die Probe umgedreht, wieder eingebaut und die Anlage erneut evakuiert für das Verdampfen des zweiten Metalls. Die Drahtstücke und das Schiffchen waren ebenfalls mit organischen Lösungsmitteln und Königswasser gereinigt worden. Zum Einsatz kamen Cu von Umicore Materials AG (1.5 mm Drahtdurchmesser) mit der Reinheit 99.999\%, Ni von Balzers (1 mm Drahtdurchmesser) mit der Reinheit 99.98\% und Pd von Ventron (1 mm Drahtdurchmesser) mit der Reinheit 99.997\%.

Nach dem Aufdampfen der Metallschichten wurden die Proben in Rohre aus SuprasilQuarzglas gelegt. Danach wurden die Rohre in einen auf die vorgesehene Diffusionstemperatur vorgeheizten Horizontalofen der Fa. Carbolite geschoben. Die Temperatur am Ort der Probe wurde mit Hilfe eines PtRh13-Pt-Thermoelementes kontrolliert. Die Diffusionsglühung wurde durch schnelles Herausziehen des Rohres aus dem Ofen und anschließende Abkühlung an Luft beendet. Die Abkühlrate im Temperaturbereich direkt unterhalb der Diffusionstemperatur betrug dabei $-6 \mathrm{~K} / \mathrm{s}$. Zwischen dem Einlegen der Probe in das Rohr und dem Hineinschieben des Rohres in den Ofen sowie während der gesamten Glühung wurde das Rohr mit Ar der Reinheit 99.9999\% als Schutzgas gespült. Für jedes Metall und jede Kombination von Metallen war ein eigenes Rohr vorgesehen, das jeweils vor der ersten Benutzung in einer Wanne mit dem alkalischen Reinigungsmittel Extran sowie mit verdünnter Flusssäure gereinigt worden war.

Die Präparation erfolgte stets nach dem oben beschriebenen Schema. Die experimen-

\begin{tabular}{|c||c|c|c|c|c|c|c||c|}
\hline Probe & Material & $\begin{array}{c}\text { Metall } \\
\text { Of- } 1\end{array}$ & $\begin{array}{c}\mathrm{d}_{1} \\
\mathrm{~nm}\end{array}$ & $\begin{array}{c}\text { Metall } \\
\text { Of-2 }\end{array}$ & $\begin{array}{c}\mathrm{d}_{2} \\
\mathrm{~nm}\end{array}$ & $\begin{array}{c}\mathrm{T}_{D} \\
{ }^{\circ} \mathrm{C}\end{array}$ & $\begin{array}{c}\mathrm{t}_{D} \\
\mathrm{~min}\end{array}$ & $\begin{array}{c}\text { Ergebnisse } \\
\text { in Abschnitt }\end{array}$ \\
\hline 1 & Bikristall & $\mathrm{Cu}$ & 140 & - & - & 1050 & 30 & 4.1 \\
\hline 2 & Bikristall & - & - & $\mathrm{Cu}$ & 190 & 1050 & 30 & 4.1 \\
\hline 3 & Bikristall & $\mathrm{Ni}$ & 45 & $\mathrm{Cu}$ & 200 & 1050 & 30 & 4.2 .1 \\
\hline 4 & Bikristall & $\mathrm{Ni}$ & 30 & $\mathrm{Cu}$ & $0 / 220$ & 900 & 1.5 & 4.2 .2 \\
\hline 5 & Bikristall & - & - & $\mathrm{Pd}$ & 14 & 1050 & 30 & 4.3 \\
\hline 6 & Bikristall & $\mathrm{Ni}$ & 30 & $\mathrm{Pd}$ & 8.7 & 1050 & 30 & 4.3 \\
\hline 7 & Fz-Si & $\mathrm{Ni}$ & 30 & $\mathrm{Pd}$ & 8.7 & 1050 & 30 & 4.3 .3 \\
\hline
\end{tabular}

Tabelle B.1. - Experimentelle Parameter der Diffusionsproben. Die Metallschichten der Dicke $d_{1}$ und $d_{2}$ wurden auf gegenüberliegende Oberflächen Of-1 und Of-2 der Proben aufgedampft, vgl. Abb. A.1. Im Fall der Probe 4 blieb eine Hälfte der Probe von $\mathrm{Cu}$ unbedeckt. Die Diffusionsglühung bei Temperatur $T_{D}$ und Dauer $t_{D}$ wurde bei allen Proben durch Herausziehen aus dem Ofen beendet, was einer Abkühlrate von $6 \mathrm{~K} / \mathrm{s}$ entspricht. 
tellen Parameter einzelner Proben wie beispielsweise die Dicke der aufgedampften Metallschicht oder die Temperatur und Dauer der Diffusionsglühung lassen sich der Tabelle B.1 entnehmen.

Bei Proben mit $\mathrm{Cu}$ als einziger metallischer Verunreinigung wurde die $\mathrm{Cu}$-Schicht auf unterschiedlichen Seiten des Bikristalls aufgebracht, wobei A und B die beiden Scheiben bezeichnen, aus denen die Probe besteht und die sich hinsichtlich der Anwesenheit von Mikrodefekten unterscheiden, vgl. Anhang A. Bei Probe 4 wurde die Cu-Schicht nur auf einer Hälfte der Probe aufgebracht, vgl. Abb. 4.16, um lateral ein Konzentrationsprofil einzustellen. Alle Diffusionsproben außer der Probe 7 wurden aus dem bikristallinen CzSi präpariert. Für Probe 7 wurde im Zonenschmelzverfahren hergestelltes defektfreies Si (Fz-Si) benutzt.

\section{B.2. Präparation von Querschnittsproben}

\section{B.2.1. Proben für Ätzgrübchenuntersuchung}

Für Ätzgrübchenuntersuchungen wurden zunächst $3 \times 3 \times 1.3 \mathrm{~mm}^{3}$ große Teile der Diffusionsproben abgebrochen und mit gleichgroßen Teilen aus defektfreiem und nicht mit Metallen kontaminiertem Fz-Silizium zusammengeklebt. Dieses geschah, um bei der lichtmikroskopischen Untersuchung nach der Defektätzung durch Ausscheidungen oder andere Defekte induzierte Ätzgrübchen von Präparationsartefakten unterscheiden zu können.

Aus den zusammengeklebten Teilen wurden nun mit einer Diamantdrahtsäge (Fa. Well) Streifen so ausgesägt, dass die Sägeflächen $\{110\}$-orientiert waren. Die Proben wurden um $90^{\circ}$ gedreht und mit Thermoplast Lakeside-Brand mit einer Sägefläche nach unten auf einen Glasträger geklebt, mit Schleifpapier aus Si-Carbid plan geschliffen und mit Diamantpasten unterschiedlicher Körnung $(7 \mu \mathrm{m}, 3 \mu \mathrm{m}, 1 \mu \mathrm{m}, 0.25 \mu \mathrm{m})$ poliert. Nach der Politur wurde die Probe vom Glasträger abgelöst und in einem auf $130{ }^{\circ} \mathrm{C}$ erhitzten Bad aus Dimethylformamid von Resten des Thermoplast gesäubert.

Für die Defektätzung kam die Secco-Lösung [91] zum Einsatz, die eine Mischung aus 49\%-iger Flussäure und einer 0.15-molaren Kaliumdichromatlösung $\left(\mathrm{K}_{2} \mathrm{Cr}_{2} \mathrm{O}_{7}\right)$ im Verhältnis 2:1 ist. Nach Befüllen des Ätzbechers mit $6 \mathrm{ml}$ Flusssäure, wurde zunächst die Probe in den Becher gelegt. Durch die Zugabe von $3 \mathrm{ml}$ der Kaliumdichromatlösung beginnt der defektselektive Ätzvorgang, der in dieser Arbeit bereits nach 5 bis 6 Sekunden durch Zusatz von deionisiertem Wasser beendet wurde. Um sukzessive die Lösung zu verdünnen, wurde der Ätzbecher mehrfach abgeschüttet und wieder mit Wasser aufgefüllt. Zuletzt erfolgte noch die Zugabe einiger Tropfen Flusssäure, um Rückstände von Siliziumdioxid an der Probenoberfläche zu entfernen. Danach fand erneut eine mehrfache Verdünnung der Lösung im Ätzbecher durch Zugabe von Wasser statt, bevor die 
Probe schließlich entnommen wurde. Die vor dem Ätzen polierte Oberfläche liegt parallel zu einer $\{110\}$-orientierten Sägefläche, so dass Ätzgrübchen auf dieser Oberfläche auf Defekte im Querschnitt der Diffusionsprobe hinweisen. Die Lage der Korngrenze der bikristallinen Proben beispielsweise wird durch einen durch die gesamte Probe laufenden Graben angezeigt.

\section{B.2.2. TEM-Proben}

Die Präparation von Querschnittsproben für TEM verlief zunächst analog zur Präparation der Proben für Ätzgrübchenuntersuchungen und folgte damit einem beispielsweise von Seibt [50] oder Riedel [90] benutzten Standardverfahren. Anstelle des Defektätzens fand nach der Politur der Vorderseite eine mechanische Dünnung durch Schleifen und Polieren der Probe von der Rückseite her statt, bis die Probe lediglich noch etwa 20 $\mu \mathrm{m}$ dick war. Nach Befestigung auf einem TEM-Netzchen aus Gold erfuhren die Proben einen Beschuss mit Ar-Ionen in einer Ionenätzanlage der Fa. Bal-Tec (Modell RES 010). Der Beschuss erfolgte unter einem Winkel von $17^{\circ}$ bei einer Spannung von $4.5 \mathrm{kV}$, wobei die Probe in einem Sektor mit $20^{\circ}$ Öffnungswinkel gedreht wurde, bis sich ein in der Mitte der Querschnittsprobe in der Nähe der Korngrenze ein Loch bildete, an dessen Rand die Probe elektronentransparent ist.

Die oben geschilderte konventionelle Präparation ermöglicht die elektronenmikoroskopische Untersuchung eines relativ großen Bereiches der Diffusionsprobe. Jedoch ist es bei sehr geringen Defektdichten wie im Fall einzelner isolierter Ausscheidungen unter nickelreichen Bedingungen in dieser Arbeit, vgl. Abschnitt 4.2.2, notwendig, einzelne Defekte gezielt für TEM zu präparieren. Dafür wurde in dieser Arbeit ein kürzlich von Stolze [77] vorgestelltes Verfahren benutzt, das Defektätzen und die Präparation mit einem fokussierten Ionenstrahl (FIB) kombiniert. Mit einem Rasterelektonenmikroskop (SEM) wurde an der Oberfläche einer defektgeätzten Querschnittsprobe eine Stelle, an der Nireiche Ausscheidungen vorliegen, anhand des Ätzgrübchens lokalisiert. An dieser Stelle ließs sich dann mittels FIB eine zur Oberfläche der Querschnittsprobe senkrecht stehende Lamelle ausschneiden und auf einen für den Einbau ins TEM geeignetes Träger aus Molybdän anbringen. Schließlich erfolgte noch eine Dünnung durch Ga-Ionenbeschuss, bis die Lamelle eletronentransparent wurde. 


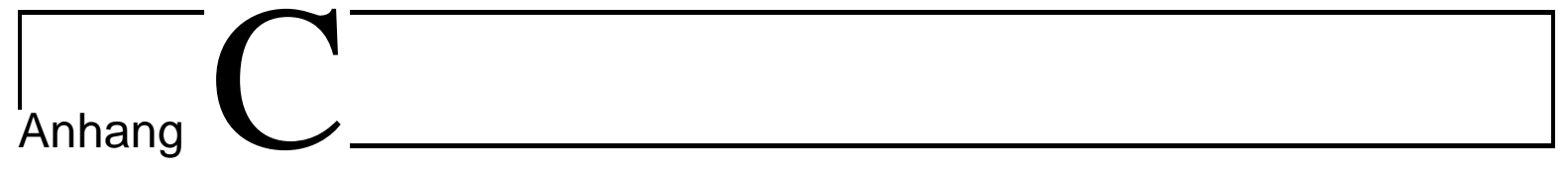

\section{Volumenfehlpassung bei Vorliegen zweier metallischer Verunreinigungen}

Es wird der Fall betrachtet, dass zwei metallische Verunreinigungen $M 1$ und $M 2$ gleichzeitig in Silizium vorliegen und Ausscheidungen zweier verschiedener Phasen bilden. Die Phasen lassen sich nominell als Silizide der Zusammensetzung $M 1_{x_{r}} M 2_{y_{r}} S i_{z_{r}}$ beschreiben, wobei $r=1,2$. Die Parameter $x_{1}$ bis $z_{2}$ geben die Anzahl der $M 1-, M 2-$ und Si-Atome pro Formeleinheit der jeweiligen Phase an. Für die in dieser Arbeit untersuchten Metalle $\mathrm{Cu}$ und $\mathrm{Ni}$ sind in Tabelle C.1 Werte angegeben.

Wenn Si-Atome in den Phasen jeweils ein anderes spezifisches Volumen als in der Si-Matrix haben, ist das Wachstum der Ausscheidungen mit einer Volumenänderung $\Delta V=V_{P}^{\text {tot }}-V_{S i}^{\text {tot }}$ verbunden. $V_{P}^{\text {tot }}$ ist das Volumen aller Ausscheidungen beider Phasen und $V_{S i}^{t o t}$ das Volumen, das alle in den Ausscheidungen enthaltenen Si-Atome zusammen in der Si-Matrix einnehmen. Diese Volumen kann durch eine Summe über die beteiligten Phasen ausgerückt werden:

$$
V_{S i}^{t o t}=\sum_{r} N_{r} z_{r} \Omega
$$

wobei $N_{r}$ die Anzahl der ausgeschiedenen Formeleinheiten der Phase $r$ angibt und $\Omega$ das Volumen pro Si-Atom in der Matrix bezeichnet.

Für den Fall, dass die Ausscheidungen die Volumenänderung $\Delta_{V}$ durch die Erzeugung intrinsischer Punktdefekte des Si kompensieren, lässt sich $\Delta_{V}$ analog zur binären Metallsilizidausscheidung darstellen, vgl. Abschnitt 2.1.2.1. 


$$
\Delta V=\sum_{r} N_{r}\left(x_{r} \alpha_{r 1}+y_{r} \alpha_{r 2}\right) \Omega
$$

Die Parameter $\alpha_{r 1}$ und $\alpha_{r 2}$ geben dabei die Differenz der interstiellen Si-Atome und Leerstellen an, die pro Ausscheidung eines M1- bzw. eines M2-Atoms in der Phase $r$ emittiert werden. Die Beiträge beider metallischer Verunreinigungen zu $\Delta V$ werden über die beteiligten Phasen aufsummiert.

Der Beitrag einer einzelnen ternären Phase zur Volumenänderung $\Delta_{V}$ insgesamt ist mit der jeweils ausgeschiedenen Menge gewichtet. Diese Gewichtung lässt sich durch eine mittlere Volumenfehlpassung ausdrücken, die hier definiert wird als:

$$
\begin{aligned}
\delta_{V}^{a v} & =\frac{V_{P}^{t o t}-V_{S i}^{t o t}}{V_{S i}^{t o t}} \\
& =\frac{\sum_{r} N_{r}\left(x_{r} \alpha_{r 1}+y_{r} \alpha_{r 2}\right)}{\sum_{r} N_{r} z_{r}},
\end{aligned}
$$

und die als Funktion des Konzentrationsverhältnisses $Q_{1,2}$ der beiden metallischen Verunreinigungen

$$
Q_{1,2}=\frac{[M 1]}{[M 2]}=\frac{\sum_{r} x_{r} N_{r}}{\sum_{r} y_{r} N_{r}}
$$

dargestellt werden kann. Für die mittlere Volumenfehlpassung ergibt sich so der Ausdruck:

$$
\delta_{V}^{a v}=\frac{A\left(x_{1} \alpha_{11}+y_{1} \alpha_{12}\right)+B\left(x_{2} \alpha_{21}+y_{2} \alpha_{22}\right)}{A z_{1}+B z_{2}}
$$

wobei

$$
\begin{aligned}
A & =x_{2}-y_{2} Q_{1,2} \\
B & =y_{1} Q_{1,2}-x_{1}
\end{aligned}
$$

Für das in dieser Arbeit untersuchte System Cu-Ni-Si wird die mittlere Volumenfehl- 


\begin{tabular}{|c|c|c|c|c|c|}
\hline \multirow[t]{3}{*}{ Parameter } & \multirow[t]{3}{*}{ Komponente } & \multirow[t]{3}{*}{ Phase } & \multicolumn{3}{|c|}{ Wert } \\
\hline & & & \multicolumn{2}{|c|}{ ternär } & \multirow{2}{*}{ binär } \\
\hline & & & $\mathrm{Cu}_{\mathrm{Si}}$ & $\mathrm{Cu}_{\mathrm{Ni}}$ & \\
\hline$x_{1}$ & $\mathrm{Cu}$ & $\mathrm{Cu}_{3} \mathrm{Si}: \mathrm{Ni}$ & \multirow{3}{*}{\multicolumn{2}{|c|}{$\begin{array}{c}3 X_{C u}^{(P)} \\
1-X_{C u}^{(P)} \\
1\end{array}$}} & 3 \\
\hline$y_{1}$ & $\mathrm{Ni}$ & $\mathrm{Cu}_{3} \mathrm{Si}: \mathrm{Ni}$ & & & 0 \\
\hline$z_{1}$ & $\mathrm{Si}$ & $\mathrm{Cu}_{3} \mathrm{Si}: \mathrm{Ni}$ & & & 1 \\
\hline$x_{2}$ & $\mathrm{Cu}$ & $\mathrm{NiSi}_{2}: \mathrm{Cu}$ & $\frac{X_{C u}^{(p)}}{1-X_{C}^{(p)}}$ & $X_{C u}^{(p)}$ & 0 \\
\hline$y_{2}$ & $\mathrm{Ni}$ & $\mathrm{NiSi}_{2}: \mathrm{Cu}$ & 1 & $1-X_{C u}^{(p)}$ & 1 \\
\hline$z_{2}$ & $\mathrm{Si}$ & $\mathrm{NiSi}_{2}: \mathrm{Cu}$ & $\frac{2-3 X_{C u}^{(p)}}{1-X_{C)}^{(p)}}$ & 2 & 2 \\
\hline
\end{tabular}

Tabelle C.1. - Die Parameter $x_{1}$ bis $z_{2}$ bezeichnen die Anzahl der Cu-, Ni- und Si-Atome pro Formeleinheit der Phasen $\mathrm{Cu}_{3} \mathrm{Si}: \mathrm{Ni}$ und $\mathrm{NiSi}_{2}: \mathrm{Cu}$, ausgedrückt durch den partiellen $\mathrm{Mo}-$ lenbruch $X_{C u}^{(P)}$ von $\mathrm{Cu}$. Im ternären System hängen die Werte für die $\mathrm{NiSi}_{2}: \mathrm{Cu}$-Phase vom Gitterplatz des $\mathrm{Cu}$ ab. Die Werte in Spalte $\mathrm{Cu}_{\mathrm{Si}}$ ergeben sich für $\mathrm{Cu}$ auf einem Si-Platz, die Werte in Spalte $\mathrm{Cu}_{\mathrm{Ni}}$ für $\mathrm{Cu}$ auf einem Ni-Platz.

\begin{tabular}{|c|c|c|c|c|c|}
\hline \multirow[t]{3}{*}{ Parameter } & \multirow[t]{3}{*}{ Komponente } & \multirow[t]{3}{*}{ Phase } & \multicolumn{3}{|c|}{ Wert } \\
\hline & & & \multicolumn{2}{|c|}{ ternär } & binär \\
\hline & & & $\mathrm{Cu}_{\mathrm{Si}}$ & $\mathrm{Cu}_{\mathrm{Ni}}$ & \\
\hline$\alpha_{11}$ & $\mathrm{Cu}$ & $\mathrm{Cu}_{3} \mathrm{Si}: \mathrm{Ni}$ & \multicolumn{2}{|c|}{0.5} & 0.5 \\
\hline$\alpha_{12}$ & $\mathrm{Ni}$ & $\mathrm{Cu}_{3} \mathrm{Si}: \mathrm{Ni}$ & \multicolumn{2}{|c|}{0.5} & 0 \\
\hline$\alpha_{21}$ & $\mathrm{Cu}$ & $\mathrm{NiSi}_{2}: \mathrm{Cu}$ & 1.0 & -0.02 & 0 \\
\hline$\alpha_{22}$ & $\mathrm{Ni}$ & $\mathrm{NiSi}_{2}: \mathrm{Cu}$ & -0.02 & -0.02 & -0.02 \\
\hline
\end{tabular}

Tabelle C.2. - Die Werte der Parameter $\alpha_{11}$ bis $\alpha_{22}$ geben die Differenz der interstiellen Si-Atome und Leerstellen an, die pro Ausscheidung eines Cu- oder Ni-Atoms in einer der Phasen $\mathrm{Cu}_{3} \mathrm{Si}: \mathrm{Ni}$ und $\mathrm{NiSi}_{2}: \mathrm{Cu}$ emittiert werden. Im ternären System hängen die Werte im Fall der $\mathrm{NiSi}_{2}$ :Cu-Phase vom Gitterplatz des $\mathrm{Cu}$ ab, $\mathrm{Cu}_{\mathrm{Si}}$ : $\mathrm{Cu}$ auf einem Si-Platz, $\mathrm{Cu}_{\mathrm{Ni}}: \mathrm{Cu}$ auf einem Ni-Platz. Die Zahlen in der Spalte „binär" geben die Werte, die sich bei Raumtemperatur für $\mathrm{NiSi}_{2}$ und $\mathrm{Cu}_{3} \mathrm{Si}$ aus der Literatur ergeben [48].

passung $\delta_{V}^{a v}$ bei Anwesenheit von $\mathrm{Cu}_{3} \mathrm{Si}: \mathrm{Ni}-$ und $\mathrm{NiSi}_{2}: \mathrm{Cu}$-Ausscheidungen mit den in Tab. C.1 und Tab. C.2 angegebenen Parametern berechnet. Die genaue Zusammensetzung der Phasen hängt vom partiellen Molenbruch $X_{C u}^{(P)}$ und außerdem vom Gitterplatz der gelösten Verunreinigung ab. Abb 5.1 in Abschnitt 5.2.2.2 der Diskussion zeigt den Verlauf, der sich für drei verschiedene Fälle

- $\mathrm{Cu}_{3} \mathrm{Si}: \mathrm{Ni}-$ und $\mathrm{NiSi}_{2}: \mathrm{Cu}-$ Ausscheidungen mit $\mathrm{Cu}$ auf Si-Plätzen

- $\mathrm{Cu}_{3} \mathrm{Si}: \mathrm{Ni}-$ und $\mathrm{NiSi}_{2}$ :Cu-Ausscheidungen mit $\mathrm{Cu}$ auf Ni-Plätzen

- binäre $\mathrm{Cu}_{3} \mathrm{Si}$ - und $\mathrm{NiSi}_{2}$-Ausscheidungen ohne ternäre Löslichkeit 


\section{Literaturverzeichnis}

[1] R. M. Swanson, A vision for crystalline silicon photovoltaics, Prog. Photovolt: Res. Appl. 14, 443 (2006).

[2] J. G. Wilkes, K. E. Benson und W. Lin, Handbook of Semiconductor Technology, Volume 2, Kapitel Silicon Pocessing, Seiten 1-66, Wiley-VCH, 2000.

[3] W. Bergholz und D. Gilles, Impact of Research on Defects in Silcon on the Microelectronic Industry, phys. stat. sol. (b) 222, 5 (2000).

[4] D. Gilles, E. R. Weber und S. Hahn, Mechanism of internal gettering of interstitial impurities in Czochralski-grown silicon, Phys. Rev. Lett. 64, 196 (1990).

[5] T. Buonassisi, A. A. Istratov, M. D. Pickett, M. Heuer, J. Kalejs, G. Hahn, M. A. Marcus, B. Lai, Z. Cai, S. M. Heald, R. F. Clark, D. W. Cunningham, A. M. Gabor, R. Jonczl, S. Narayanan, E. Sauar und E. R. Weber, Chemical Natures and Distribution of Metal Impurities in Multicrystalline Silicon Materials, Prog. Photovolt: Res. Appl. 14, 513 (2006).

[6] H. J. Möller, L. Long, M. Werner und D. Yang, Oxygen and Carbon Precipitation in Multicrystalline Solar Silicon, phys. stat. sol. (a) 171, 175 (1999).

[7] A. A. Istratov, T. Buonassisi, R. J. McDonald, A. R. Smith, R. Schindler, J. A. Rand, J. P. Kalejs und E. R. Weber, Metal content of multicrystalline silicon for sola cells and its impact on minority carrier diffusion length, J. Appl. Phys. 94, 6552 (2003).

[8] J. W. Chen und A. G. Milnes, Energy Levels in Silicon, Ann. Rev. Mater. Sci. 10, 157 (1980).

[9] R. N. Hall, Electron-Hole Recombination in Germanium, Phys. Rev. 87, 387 (1952). 
[10] W. Shockley und W. T. Read, Statistics of the Recombinations of Holes and Electrons, Phys. Rev. 87, 835 (1952).

[11] M. Kittler, J. Lärz, W. Seifert, M. Seibt und W. Schröter, Recombination properties of structurally well defined NiSi2 precipitates in silicon, Appl. Phys. Lett. 58, 911 (1991).

[12] W. Schröter, V. Kveder, M. Seibt, H. Ewe, H. Hedemann, F. Riedel und A. Sattler, Atomic structure and electronic states of nickel and copper silicides in silicon, Mat. Sci. Eng. B 72, 80 (2000).

[13] C. P. Ewels, S. Leoni, M. I. Heggie, P. Jemmer, E. Hernández, R. Jones und P. R. Briddon, Hydrogen Interaction with Dislocations in Si, Phys. Rev. Lett. 84, 690 (2000).

[14] M. Seibt, V. Kveder, W. Schröter und O. Voß, Structural and electrical properties of metal impurities at dislocations in silicon, phys. stat. sol. (a) 202, 911 (2005).

[15] M. Kittler, W. Seifert und V. Higgs, Recombination Activity of Misfit Dislocations in Silicon, phys. stat. sol. (a) 137, 327 (1993).

[16] P. R. Wilshaw und T. S. Fell, Electron Beam Induced Current Investigations of Transition Metal Impurities at Extended Defects in Silicon, J. Electrochem. Soc. 142, 4298 (1995).

[17] V. Kveder, M. Kittler und W. Schröter, Recombination activity of contaminated dislocations in silicon: A model describing electron-beam-induced current contrast behavior, Phys. Rev. B 63, 115208 (2001).

[18] J. Chen, T. Sekiguchi, D. Yang, F. Yin, K. Kido und S. Tsurekawa, Electron-beaminduced current study of grain boundaries in multicrystalline silicon, J. Appl. Phys. 96, 5490 (2004).

[19] J. Chen, T. Sekiguchi, R. Xie, P. Ahmet, T. Chikyo, D. Yang, S. Ito und F. Yin, Electron-beam-induced current study of small-angle grain boundaries in multicrystalline silicon, Scripta Materialia 52, 1211 (2005).

[20] T. Buonassisi, A. A. Istratov, M. D. Pickett, M. A. Marcus, T. F. Ciszek und E. R. Weber, Metal precipitation at grain boundaries in silicon: Dependence on grain boundary character and dislocation decoration, Appl. Phys. Lett. 89, 042102 (2006).

[21] S. M. Myers, M. Seibt und W. Schröter, Mechanisms of transition-metal gettering in silicon, J. Appl. Phys. 88, 3795 (2000). 
[22] T. Buonassisi, A. A. Istratov, M. A. Marcus, B. Lai, Z. Cai, S. M. Heald und E. R. Weber, Engineering metal-impurity nanodefects for low-cost solar cells, Nature Materials 4, 676 (2005).

[23] N. Yuge, M. Abe, K. Hanazawa, H. Baba, N. Nakamura, Y. Kato, Y. Sakaguchi, S. Hiwasa und F. Aratani, Purification of metallurgical-grade silicon up to solar grade, Prog. Photovolt 9, 203 (2001).

[24] A. A. Istratov, T. Buonassisi, M. D. Pickett, M. Heuer und E. Weber, Control of metal impurities in "dirty"multicrystalline silicon for solar cells, Mat. Sci. Eng. B 134, $282(2006)$.

[25] M. Seibt und K. Graff, Characterization of haze-forming precipitates in silicon, J. Appl. Phys. 63, 4444 (1988).

[26] K. Graff, Metal Impurities in Silcon-Device Fabrication, Springer, 1995.

[27] W. Schröter, M. Seibt und D. Gilles, Handbook of Semiconductor Technology, Volume 1, Kapitel High-Temperature Properties of Transion Elenments in Silicon, Seiten 598-660, Wiley-VCH, 2000.

[28] M. Heuer, T. Buonassisi, M. A. Marcus, A. A. Istratov, M. D. Pickett, T. Shibata und E. R. Weber, Complex intermetallic phase in multicrystalline silicon doped with transition metals, Phys. Rev. B 73, 235204 (2006).

[29] T. Buonassisi, M. Heuer, A. A. Istratov, M. D. Pickett, M. A. Marcus, B. Lai, Z. Lai, S. Heald und E. Weber, Transition metal co-precipitation mechanisms in silicon, Acta Materialia 55, 6119 (2007).

[30] A. G. Cullis und L. E. Katz, Electron microscope study of electrically active impurity precipitate defects in silicon, Phil. Mag. 30, 1419 (1974).

[31] K. Ryoo, R. Drosd und W. Wood, Analysis of plate and colony precipitates decorating stacking faults in a single-crystal silicon, J. Appl. Phys. 63, 4440 (1988).

[32] C. Colliex, J. L. Maurice und D. Ugarte, Frontiers of nalytical electron microscopy with special reference to cluster and interface problems, Ultramic. 29, 31 (1989).

[33] M. Reiche, K. Scheerschmidt, D. Conrad, E. Scholz, A. Plößl, U. Gösele und K. Tu, Dislocation structrue in interfaces of bonded hydrophobic silicon wafers: experiment and molecular dynamics, Inst. Phys. Conf. Ser. 157, 447 (1997).

[34] E. M. Sokolovskaya, O. I. Chechernikova, E. I. Gladyshevskiy und O. I. Bodak, The Ni-Cu-Si System, Russian Metallurgy 6, 114 (1973). 
[35] J. Utzig, An elastic energy approach to the interstitial diffusion of $3 d$ elements in silicon, J. Appl. Phys. 65, 3868 (1989).

[36] R. N. Hall und J. H. Racette, Diffusion and Solubility of Copper in Extrinsic and Intrinsic Germanium, Silicon, and Gallium Arsenide, J. Appl. Phys. 35, 379 (1964).

[37] M. K. Bakhadyrkhanov, S. Zainabidov und A. Khamidov, Some characteristics of diffusion and electrotransport of nickel in silicon, Sov. Phys. Semicon. 14, 243 (1980).

[38] K. Graff, H. A. Heffner und H. Pieper, Palladium-Test: A Tool to Evaluate Gettering Efficiency, Mater. Res. Soc. Proc. 36, 19 (1985).

[39] E. R. Weber, Transition Metals in Silicon, Appl. Phys. A 30, 1 (1983).

[40] W. Frank, The interplay of solute- and self-diffusion - a key for revealing diffusion mechanisms in silicon and germanium, Defect Diffusion Forum 75, 121 (1991).

[41] B. Tuck, Intoduction to diffusion in semiconductors, Kapitel Semiconductor solutions, Seiten 65-70, Institution of Electrical Engineers, 1974.

[42] E. Nes und J. Washburn, Precipitation in High-Purity Silicon Single Crystals, J. Appl. Phys. 42, 3562 (1971).

[43] J. K. Solberg und E. Nes, On the micromechanisms of repeated precipitation on edge dislocations, J. Mater. Sci. 13, 2233 (1978).

[44] M. Seibt, M. Griess, A. Istratov, H. Hedemann, A. Sattler und W. Schröter, Formation and Properties of Copper Silicide Precipitates in Silicon, phys. stat. sol. (a) 166, 171 (1998).

[45] P. D. Augustus, J. Knights und L. W. Kennedy, A correlation between the electrical breakdown of silicon bipolar transistors and impurity precipitates, J. Microsc. 118, 315 (1980).

[46] B. P. R. Marioton und U. Gösele, Transport of thermodynamic information of self-interstitials between precipitates in silicon, J. Appl. Phys. 63, 4661 (1988).

[47] H. Vogel, Gerthsen Physik, Seite 211, Springer, 1995.

[48] S. P. Murarka, Silicides for VLSI Applications, Kapitel Properties, Seiten 29-70, Academic Press Inc., 1983.

[49] H. Ewe, Semiconductor 1994, Seite 796, The Electrochemical Society, 1994. 
[50] M. Seibt, Elektronenmikroskopische Untersuchung des Ausscheidungsverhaltens von Nickel in Silizium, Dissertation, Göttingen, 1986.

[51] M. Seibt und W. Schröter, Precipiatation behaviour of nickel in silicon, Phil. Mag. A 59, 337 (1989).

[52] R. T. Tung, J. M. Gibson und J. M. Poate, Formation of Ultrathin Single-Crystal Silicide Films on Si: Surface and Interfacial Stabilization of Si-NiSi $i_{2}$ Epitaxial Structures, Phys. Rev. Lett. 50, 429 (1983).

[53] J. K. Solberg, The Crystal Structure of $\eta-C u_{3}$ Si Precipitates in Silicon, Acta Cryst. A34, 684 (1978).

[54] K. Anderko und K. Schubert, Kristallstruktur von $\mathrm{Pd}_{2} \mathrm{Si}, \mathrm{Pd}_{2} G e$ und $\mathrm{Pt}_{2} \mathrm{Ge}, \mathrm{Z}$. Metallk. 44, 307 (1953).

[55] J. M. Silcock und W. J. Tunstall, Partial Dislocations Associated with NbC Precipiatation in Austenitic Stainless Steels, Phil. Mag. 10, 361 (1964).

[56] E. Nes, Mechanism of Repeated Precipiatation on Dislocations, Acta Metall. 22, 81 (1974).

[57] D. West, Ternary Equilibrium Diagrams, Kapitel General Considerations, Seiten 1-10, Chapman and Hall, 1982.

[58] M. Setton, Properties of Metal Silicides EMIS Datareview Series No.14, Kapitel 3.7 Ternary TM-TM-Si reactions, Seiten 129-149, INSPEC, 1995.

[59] P. Villars, A Semiempirical approach to the prediction of compound formation for 96446 ternary alloys systems: II, J. Less-Common Met. 119, 175 (1986).

[60] M. Setton und J. van der Spiegel, Structural and electrical properties of $\mathrm{ZrSi}_{2}$ and $\mathrm{Zr}_{2} \mathrm{CuSi}_{4}$ formed by rapid thermal processing, J. Appl. Phys 70, 193 (1991).

[61] C. Fetzer, I. Dezsi, A. Vantomme, M. Wu, S. Jin und H. Bender, Ternary $\mathrm{Co}_{x} \mathrm{Fe}(1-x) \mathrm{Si}_{2}$ and $\mathrm{Ni} i_{x} \mathrm{Fe}(1-x) \mathrm{Si}_{2}$ formed by ion implantation in silicon, J. Appl. Phys. 92, 3688 (2002).

[62] T. G. Finstad, D. K. Anfiteatro, V. R. Delfine, F. M. d' Heurle, P. Gas, V. L. Moruzzi, K. Schwarz und J. Tersoff, The Formation of Disilicides From Bilayers Of Ni/Co And Co/Ni On Silicon: Phase Separation And Solid Solution, Thin Solid Films 135, 229 (1986).

[63] M. Okamoto, The Investigation of the Equilibrium State of the Ternary Whole System Cu-Ni-Si. III. On the Heterogenous Equilibrium Relating to Liquid of the Ternary Whole System Cu-Ni-Si, J. Jpn. Inst. Met. 3, 365 (1939). 
[64] E. Jänecke, Kurzgefasstes Handbuch Aller Legierungen, Kapitel Ternäre Legierungen, Seiten 513-517, Carl Winter, Heidelberg, 1949.

[65] K. C. H. Kumar, A. Kussmaul, H. L. Lukas und G. Effenberg, Numerical data and functional relationships in science and technology: New series / Landolt-Börnstein IV/11C2, Seiten 374-381, Springer-Verlag, 2007.

[66] W. Wopersnow und K. Schubert, Über die Mischung Nickel-Palladium-Silizium, Z. Metallk. 67, 807 (1976).

[67] T. G. Finstad und M.-A. Nicolet, Silicide formation with bilayer of $P d-P t, P d-N i$, and Pt-Ni, J. Appl. Phys. 50(1), 303 (1979).

[68] M. E. Loomans, D. Z. Chi und S. J. Chua, Monosilicide-Disilicide-Silicon Phase Equilibria in the Nickel-Platinum-Silicon and Nickel-Palladium-Silicon Systems, Metall. Mater. Trans. A 35, 3053 (2004).

[69] E. L. Semenova, Numerical data and functional relationships in science and technology: New series / Landolt-Börnstein IV/11C3, Seiten 486-493, Springer, 2007.

[70] M. Heuer, T. Buonassisi, A. A. Istratov, M. D. Picket, M. A. Marcus, A. M. Minor und E. Weber, Transition metal interaction and $\mathrm{Ni}-\mathrm{Fe}-\mathrm{Cu}-\mathrm{Si}$ phases in silicon, J. Appl. Phys. 101, 123510 (2007).

[71] T. F. Ciszek und T. H. Wang, Silicon defect and impurity studies using float-zone crystal growth as a tool, J. Cryst. Growth 237-239, 1685 (2002).

[72] O. F. Vyvenko, T. Buonassisi, A. A. Istratov und E. R. Weber, X-ray beam induced current/microprobe $x$-ray fluorescence: synchrotron radiation based $x$-ray microprobe techniques for analysis of the recombination activity and chemical nature of metal impurities in silicon, J. Phys.:Condens. Matter 16, S141 (2004).

[73] M. von Heimendahl, Einführung in die Elektronenmikroskopie, Vieweg, 1970.

[74] L. Reimer, Tranmission Electron Microscopy, Springer, 1984.

[75] J. M. Spence, Experimental High-Resolution Electron Microscopy, Oxford University Press, 1988.

[76] D. B. Williams und C. B. Carter, Transmission Electron Microscopy, Plenum Press, New York, 1996.

[77] L. Stolze, Lokalisierung und Charakterisierung von Silizid-Ausscheidungen in kristallinem Silizium nach Diffusion von Nickel und Kupfer, Diplomarbeit, Göttingen, 2008. 
[78] G. Cliff und G. Lorimer, The quantitative analysis of thin specimens, J. Microscopy 103, 203 (1975).

[79] W. Schröter, M.Seibt und D. Gilles, Materials Science and Technology, Kapitel High-Temperatur Properties of 3d Transition Elements in Silicon, Seite 569, WileyVCH, 1991.

[80] W. C. Dash, Copper Precipiatation on Dislocations in Silicon, J. Appl. Phys. 27, 1193 (1956).

[81] K. Graff, Aggregation Phenomena of Point Defects in Silicon, The Electrochemical Soc., Pennington, 1983.

[82] P. Saring, C. Rudolf, L. Stolze und M. Seibt, Light-Beam Induced Current measurements on copper-nickel co-contaminated Cz-silicon bicrystals, Mat. Sci. Eng. B, angenommen (2009).

[83] N. Gay und S. Martinuzzi, External self-gettering of nickel in float zone silicon wafers, Appl. Phys. Lett. 70, 2568 (1997).

[84] D. Abdelbarey, V. Kveder, W. Schröter und M.Seibt, Aluminum gettering of iron in silicon as a problem of the ternary phase diagram, Appl. Phys. Lett., angenommen (2009).

[85] R. Wagner, R. Kampmann und P. Voorhees, Phase Transformations in Materials, Kapitel Homogeneous Second-Phase Precipitation, Seiten 322-326, Wiley-VCH, 2001.

[86] J. C. H. Spence und J. Taftø, ALCHEMI: a new technique for locating atoms in small crystals, Journal of Microscopy 130, 147 (1983).

[87] M. Reiche, Semiconductor wafer bonding, phys. stat. sol. (a) 203, 747 (2006).

[88] A. Borghesi, B. Pivac, A. Sassella und A. Stella, Oxygen precipitation in silicon, J. Appl. Phys. 77, 4169 (1994).

[89] A. Sattler, Ratenlimitierende Prozesse beim Gettern von Kobalt in Silizium, Dissertation, Göttingen, 2003.

[90] F. Riedel, Zusammenhang elektrischer und struktureller Eigenschaften von Nickelsilizidausscheidungen in Silizium, Dissertation, Göttingen, 1995.

[91] F. S. d' Aragona, Dislocation Etch for (100) Planes in Silicon, J. Electrochem. Soc. 119, 948 (1972). 


\section{Danksagung}

Ich danke meinem Doktorvater Herrn Prof. Dr. M. Seibt für die Ermöglichung dieser Arbeit, für seine stetige Diskussionsbereitschaft, seine wertvolle Kritik, Hilfe bei der Elektronenmikroskopie und die Gelegenheit zu internationalen Tagungen fahren und dort Vorträge halten zu können. Die Jahre, in denen ich in seiner Arbeitsgruppe tätig war, waren eine Erfahrung, die ich nicht missen möchte.

Herrn Prof. Dr. H. Hofsäß danke ich für die bereitwillige Übernahme des Korreferats.

Bei Herrn Prof. Dr. W. Schröter möchte ich mich besonders für inspirierende Diskussionen und Denkanstöße bedanken.

Herrn Dr. K. Ahlborn und Dr. K. Thiel sei für Hilfestellung bei Computerproblemen und Herrn Dipl.Ing. (grad.) M. Schrader für technische Hilfe gedankt.

Allen alten und neuen Mitgliedern der Arbeitsgruppe Seibt sei für das angenehme Arbeitsklima, die freundliche wohlwollende Atmosphäre gedankt, in der sich so manche Idee entwickeln konnte. Insbesondere möchte ich mich bei einer Reihe von Kollegen bedanken, mit denen zusammen ich einen langen Weg gegangen bin: Oliver Voss, Karsten Thiel, Tore Niermann und Yue-Lin Huang.

Frau Bettina Schlieper-Ludewig möchte ich für die Hilfe bei der Präparation von Proben und die vielen kleinen Hilfsdienste danken, die so oft in Vergessenheit geraten. Frau Dipl.-Phys Linda Stolze danke ich für die Präparation einer wichtigen Probe mittels FIB. Philipp Saring und Philipp Hahne, Marie Aylin Falkenberg, Doaa Abdel Barey, Oliver Voss, Niels Hildebrand, Henning Schuhmann und Hendrik Bergmann danke ich für die Korrekturen sprachlicher Fehler im Manuskript.

Ich danke meinen Eltern für die nicht nur materielle Ermöglichung des Studiums und ihnen und meiner Schwester für Zuspruch und Motivation.

Katrin Jägerfeld gebührt Anerkennung für ihre Sorge um Kilian. Meinem kleinem Sohn konnte ich während der letzten Wochen nicht viel geben, aber er gab mir um so mehr. 



\section{Lebenslauf}

Carsten Rudolf

Geburtstag:

Geburtsort:

11.10 .1975

Staatsangehörigkeit:

Familienstand:

Hameln

Deutsch

ledig, ein Sohn: Kilian Jägerfeld

1982-1986

Grundschule Fischbeck

1986-1988

Orientierungsstufe Hessisch Oldendorf

1988-1995

Schiller-Gymnasium Hameln

$1995-1996$

Zivildienst: Deutsches Rotes Kreuz, Kreisverband Hameln-Pyrmont

10/1996-05/2002

Studium der Physik an der Universität Göttingen bis zum Diplom

$05 / 2002$

Diplom

Titel der Diplomarbeit: „Reifung extrinsischer Versetzungsringe in

Silizium unter Aluminiumbedeckung“"

$05 / 2002-09 / 2005$

Fortsetzung des Studiums nach dem Diplom

$07 / 2002-12 / 2002$

Wissenschaftliche Hilfskraft der Universität Göttingen

$01 / 2003-09 / 2005$

Wissenschaftlicher Mitarbeiter des BMU-Projektes ASiS

$10 / 2005-03 / 2007$

Wissenschaftlicher Mitarbeiter des von der Volkwagenstiftung geförderten Projektes SOBSi

04/2007-12/2008

Wissenschaftlicher Mitarbeiter des BMU-Projektes SolarFocus

seit $01 / 2009$

Wissenschaftlicher Angestellter am Sonderforschungsbereich 602

der Universität Göttingen 